\title{
AN EXAMINATION OF RESPONSES TO LABORATORY STRESSORS IN INDIVIDUALS WITH BULIMIA NERVOSA COMPARED TO THOSE WITHOUT A HISTORY OF EATING DISORDERS
}

\author{
by \\ Andrea Linett \\ Master of Arts, Ryerson University, 2011 \\ Bachelor of Arts (Honours) University of Western Ontario, 2008 \\ A dissertation \\ presented to Ryerson University \\ in partial fulfillment of the \\ requirements for the degree of \\ Doctor of Philosophy \\ in the Program of \\ Psychology \\ Toronto, Ontario, Canada, 2016 \\ (C)(Andrea Linett) 2016
}




\section{AUTHOR'S DECLARATION FOR ELECTRONIC SUBMISSION OF A DISSERTATION}

I hereby declare that I am the sole author of this dissertation. This is a true copy of the dissertation, including any required final revisions, as accepted by my examiners.

I authorize Ryerson University to lend this dissertation to other institutions or individuals for the purpose of scholarly research.

I further authorize Ryerson University to reproduce this dissertation by photocopying or by other means, in total or in part, at the request of other institutions or individuals for the purpose of scholarly research.

I understand that my dissertation may be made electronically available to the public. 
An Examination of Responses to Laboratory Stressors in Individuals with Bulimia Nervosa Compared to Those Without a History of Eating Disorders

Andrea Linett

Doctor of Philosophy, 2016

Psychology

Ryerson University

\begin{abstract}
The association between perceived interpersonal stress and symptoms of bulimia nervosa (BN) has been well documented. In line with this association, recent research is focused on better understanding common factors of $\mathrm{BN}$ and social anxiety (SA; e.g., fear of negative evaluation, perfectionism) that might contribute to the maintenance of BN symptomatology. Thus far, the extent to which stress affects individuals with $\mathrm{BN}$ and co-occurring SA has not been extensively studied, despite the high comorbidity of these two disorders. Using an experimental paradigm, the present study explored the effects of different stressor-types on individuals with $\mathrm{BN}$, as well as the potential impact of SA symptoms and related factors on stress response in this population. A total of 56 females participated in this study: 28 diagnosed with $\mathrm{BN}$, and 28 controls without a history of eating disorders. All participants completed self-report measures and underwent three laboratory stressors: a modified version of the Trier Social Stress Test (a psychosocial stressor), a carbon dioxide challenge (a physiological stressor), and a body image stressor (an eating disorder specific stressor developed for use in this study). Subjective and cardiovascular responses were measured. Contrary to hypotheses, significant between-group differences in stressor response were not consistently revealed. An examination of Cohen's $d$ values, however, suggested that the magnitude of group differences represented practically significant effects.
\end{abstract}


Notably, participants within both groups reported decreased levels of hunger and desire to eat in response to each stressor. Also inconsistent with hypotheses was that neither SA symptoms nor associated factors consistently predicted stress reactivity in either group. Moreover, in the BN group, similar magnitudes of response were revealed across the three stressors. Implications and suggestions for further research are discussed. Although binge eating was not behaviourally measured, the finding that different stressor-types did not lead directly to increased hunger and desire to eat is notable and consistent with the cognitive behavioural theory of BN. With continued study of SA-related factors in association with BN symptomatology-- particularly in the context of stressful events-- further insight into the maintenance of this disorder might be revealed for a subset of individuals with comorbid BN and SAD. 


\section{Acknowledgements}

I would like to express a sincere thank you to a number of individuals who have provided me with support throughout this project. First, thank you to Dr. Kristin Vickers, my research advisor of 7 years. Kristin, you have invested countless hours into teaching me and guiding me throughout my graduate school journey. Your insight, feedback, and mentorship have greatly inspired me in this research and beyond. Thank you also to all members of the Psychophysiology Lab for contributing to a supportive and fulfilling learning environment. I would also like to thank all faculty and staff within Ryerson's Psychology Department for fostering a superb graduate program - one that I have been proud to be a part of for so many years.

I would also like to express my appreciation to the members of my supervisory committee, Dr. Martin Antony and Dr. Colleen Carney, for their guidance in the construction and completion of this project. In addition, thank you to Dr. Eric Hehman, Dr. Stephen Wonderlich, and Dr. Dale Smith for their feedback as members of my examining committee.

I must also acknowledge my family and friends for their constant support and encouragement throughout my academic endeavours. A special thank you goes out to my husband. Justin - the love, laughter and happiness that you have brought to my life over the past 6 years has been incredibly meaningful - I could not have reached my goals without you. Thank you for believing in me always. 


\section{Table of Contents}

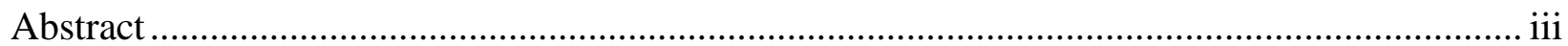

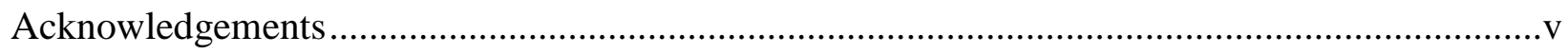

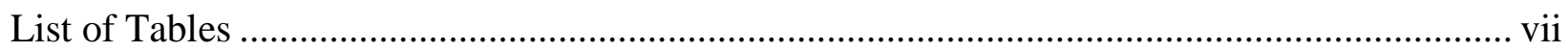

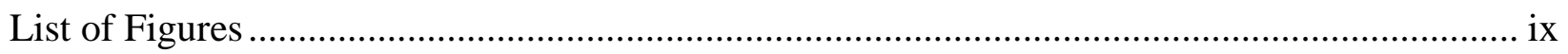

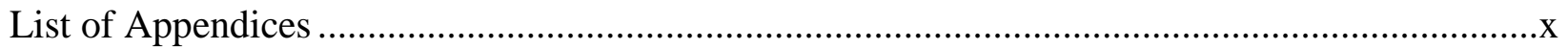

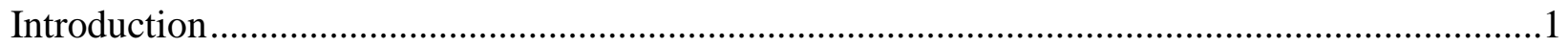

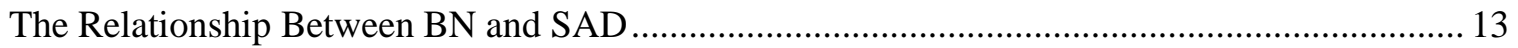

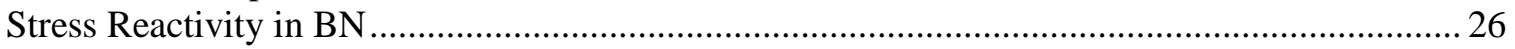

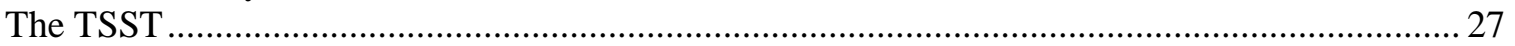

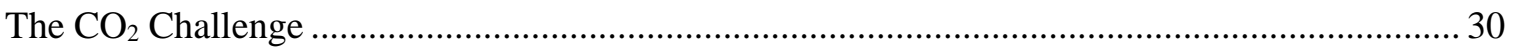

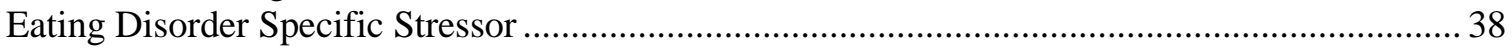

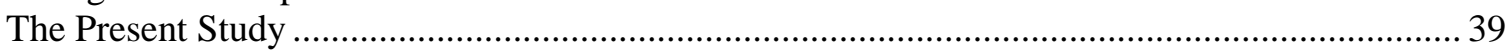

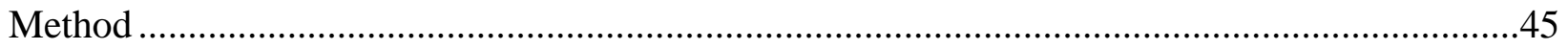

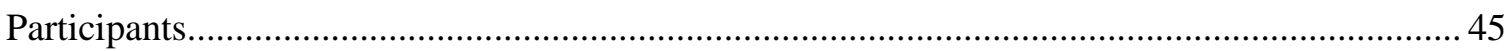

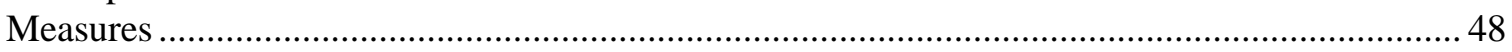

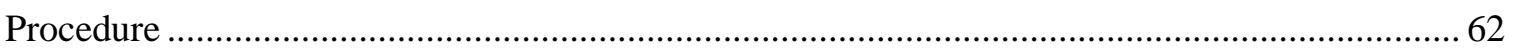

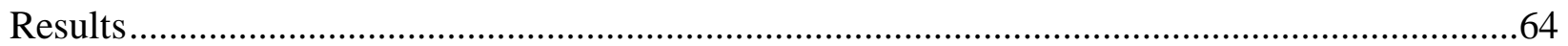

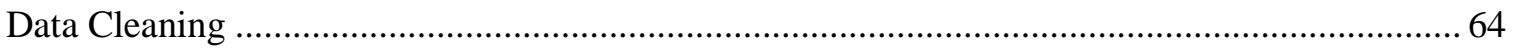

Demographics and Psychological Characteristics of the Sample ................................................ 67

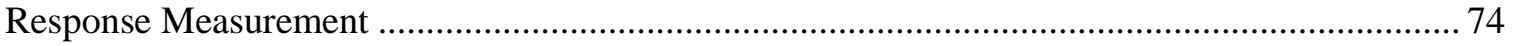

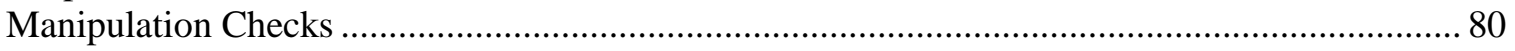

Hypothesis 1: Comparison of Response to Stressors in Participants With and Without BN......... 88

Hypothesis 2: Social Anxiety Symptoms and Social Anxiety-Related Factors as Predictors of

Stress Response in Individuals With BN and Without BN ....................................................... 95

Hypothesis 3: Associations Among Stressor Response and Psychological Symptoms (Social

Anxiety, Depression, Trait anxiety) that Commonly Co-occur with BN.................... 104

Hypothesis 4: Associations Between Stress Reactivity (Anxiety, Fear, Stress) and Body

Image/Eating Specific Responses (Desire to Eat, Hunger, Body Dissatisfaction) in BN............ 104

Hypothesis 5: Differential Stressor Effects in Individuals With BN....................................... 105

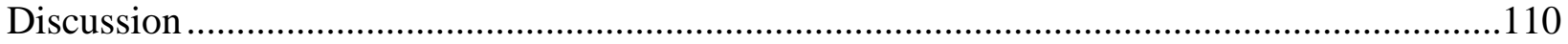

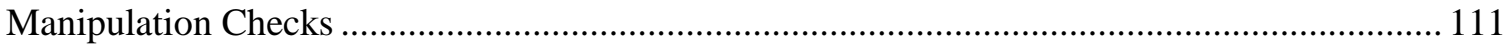

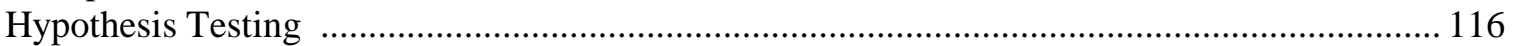

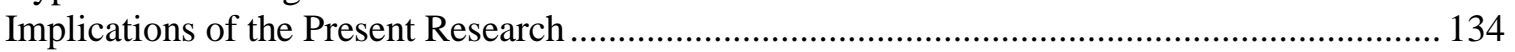

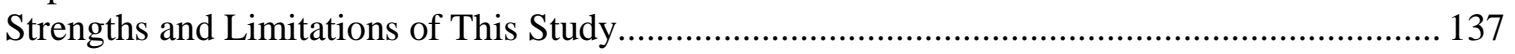

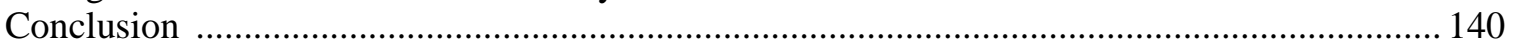

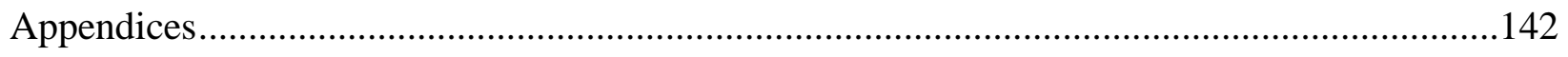

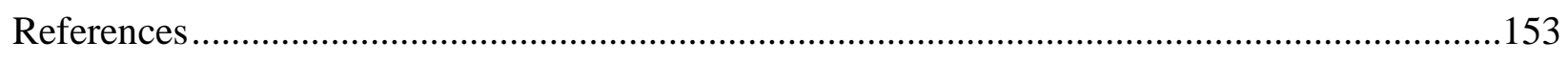

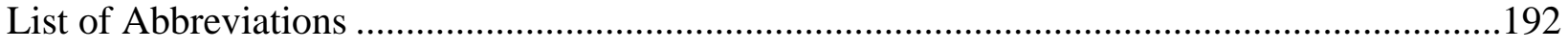




\section{List of Tables}

Table 1. Sample Demographics, Separated by Study Group.................................71

Table 2. Baseline Clinical Characteristics of the Sample, Separated by Study Group............72

Table 3. Means and Standard Deviations of Subjective Stress Response Raw Scores, Separated by Group and Stressor....................................................76

Table 4. Means and Standard Deviations of Physiological (Cardiovascular) Stress Response Measures, Separated by Group and Stressor............................................77

Table 5. Correlations Among Subjective Stress Response Indicators (Residualized Change Scores) for Each Stressor, Across Groups....................................................78

Table 6. Paired Samples T-Tests of Subjective Stress Response Raw Scores at Time 1 and Time 2, Across Groups.

Table 7. Repeated Measures Analysis of Variance of Mean HR and BP Values Measured During a Baseline Period, Stressor Task, and Recovery Period, Across Groups

Table 8. $P$-Values and Cohen's $d$ Effect Sizes for Pairwise Comparisons of Mean HR and BP Values, Across Groups.

Table 9. Paired Samples $T$-Tests of Subjective Stress Response Raw Scores at Time 1 and Time 2, Separated by Group.

Table 10. P-Values and Cohen's $d$ Effect Sizes for Pairwise Comparisons of Mean HR and BP Values from Baseline to Stressor Task, Separated by Group.

Table 11. Comparison of Subjective Responses to Each Stressor Between the BN Group and the NED Group.

Table 12. Comparison of Physiological Responses to Each Stressor Between the BN Group and the NED Group..

Table 13. Regression Analysis Predicting PSL Scores in Response to the TSST by Group, Depressive Symptoms, State Anxiety, Trait Anxiety, Anxiety Sensitivity, Perceived Stress, and Stressful Life Events....

Table 14. Regression Analyses Predicting Anxiety, Stress and Body Dissatisfaction Ratings in Response to the BIS by Group, Depressive Symptoms, State Anxiety, Trait Anxiety, Anxiety Sensitivity, Perceived Stress, and Stressful Life Events.

Table 15. Regression Analyses Predicting Anxiety, Fear, Stress, and Panic Symptoms in Response to the TSST by Social Anxiety Symptoms and Social Anxiety-Related Variables, in the BN Group. 
Table 16. Regression Analyses Predicting Anxiety, Stress, and Panic Symptoms in Response to the TSST by Social Anxiety Symptoms and Social Anxiety-Related Variables, in the NED Group

Table 17. Regression Analyses Predicting Heart Rate and Diastolic Blood Pressure in Response to the TSST by Social Anxiety Symptoms and Social Anxiety-Related Variables, Separated by

Group. .98

Table 18. Regression Analyses Predicting Fear, Panic Symptoms and Heart Rate in Response to $\mathrm{CO}_{2}$ by Social Anxiety Symptoms and Social Anxiety-Related Variables, in the BN Group. 100

Table 19. Regression Analysis Predicting Panic Symptoms in Response to $\mathrm{CO}_{2}$ by Social Anxiety Symptoms and Social Anxiety-Related Variables, in the NED Group.

Table 20. Regression Analysis Predicting DIA-BP in Response to the BIS by Social Anxiety Symptoms and Social Anxiety-Related Variables, in the BN Group...

Table 21. Regression Analysis Predicting Anxiety and DIA-BP in Response to the BIS by Social Anxiety Symptoms and Social Anxiety-Related Variables, in the NED Group 103

Table 22. Correlations ( $r$ ) Among Responses to $\mathrm{CO}_{2}$, the DASS-21-Depression and the STICSA-T, in the BN group.

Table 23. Correlations (r) Among Responses to the BIS, the DASS-21-Depression and the STICSA-T, in the BN group. 107

Table 24. Correlations (r) Among Responses to the BIS, the DASS-21-Depression and the STICSA-T, in the BN group. 108

Table 25. Repeated Measures Analysis of Variance of Within-Participant Effects of Three Stressor Tasks (Change from Time 1 to Time 2), in the BN Group. 


\section{List of Figures}

Figure 1. Moderational model of the relationship between bulimic behaviours and stress

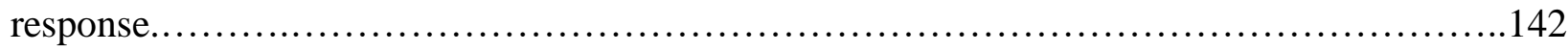




\section{List of Appendices}

Appendix A. Pilot Testing the Effects of a Newly Developed Body-Image Stressor...........142

Appendix B. Stress-Induction Studies Comparing VAS Ratings Pre and Post $\mathrm{CO}_{2} \ldots \ldots \ldots \ldots 150$

Appendix C. Effect Sizes of Reactivity Comparisons Amongst Bulimia Nervosa, Panic

Disorder, and Healthy Control Groups, Using VAS-A Change as The Outcome

Variable..................................................................... 151

Appendix D. Summary of Medical History Exclusion Criteria........................... 152 


\section{An Examination of Responses to Distinct Laboratory Stressors in Individuals with Bulimia Nervosa Compared to Those Without a History of Eating Disorders}

Bulimia nervosa $(\mathrm{BN})$ is an eating disorder characterized by recurrent episodes of uncontrolled binge eating and subsequent compensatory behaviours intended to prevent weight gain, as well as excessive concern with body weight and shape (American Psychological Association [APA], 2013). Lifetime prevalence rates for BN tend to be higher among women than men; according to the Diagnostic and Statistical Manual of Mental Disorders (DSM)-IV diagnostic criteria, approximately $1.5 \%$ of women and $0.5 \%$ of men develop BN in their lifetime, with age of onset typically in adolescence or young adulthood (Hoek \& van Hoeken, 2003; Hudson, Hiripi, Pope, \& Kessler, 2007). In a recent study of the DSM-5 diagnostic criteria, lifetime prevalence of $\mathrm{BN}$ in females by age 20 was estimated at $2.6 \%$, and the peak age of onset was between ages 16 and 20 (Stice, Marti, \& Rohde, 2013). The disorder is associated with high rates of medical complications (Mehler, 2011), psychiatric comorbidity (O'Brien \& Vincent, 2003; Wonderlich \& Mitchell, 1997), and psychosocial impairment (Kessler et al., 2014). Mortality rates, due to disorder-specific symptoms or suicide, are also significant (Arcelus, Mitchell, Wales, \& Nielsen, 2011; Crow et al., 2009).

Although psychological and pharmacological treatments are effective at reducing BN symptoms (National Institute for Clinical Excellence, 2004; Wilson, Grilo, \& Vitousek, 2007), treatment studies indicate typical outcomes associated with partial response, high dropout rates, and considerable relapse (Fichter \& Quadflieg, 2007; Herzog et al., 1999; Mitchell, Hoberman, Peterson, Mussell, \& Pyle, 1996; Shapiro et al., 2007; Stice, Marti, Shaw, \& Jaconis, 2009).

Generally, the course of the disorder is chronic and persistent, with fluctuating symptomatic and remission periods (Bohon, Stice, \& Burton, 2009; Fairburn, Cooper, Doll, Norman, \& O'Connor, 
2000). These findings, taken together, signify a clear need to enhance BN treatment efficacy. An important initial step toward this ultimate goal is to identify factors that maintain the disorder. In line with this objective, a large body of research has explored the effects of stress on BN symptoms.

Stress has been defined in a number of ways across the research literature. These definitions vary in the extent to which they emphasize stressful events, responses, or individual appraisals as the central characteristic of stress. Regardless of specific wording, all definitions emphasize the idea that stress occurs when environmental demands exceed an individual's available coping resources (Lazarus \& Folkman, 1984). This experience results in psychological and biological changes that may place an individual at risk for various illnesses over time, such as heart disease, diabetes, depression and anxiety (McEwen, 2007). The term stressor is commonly used in stress research to describe an event (a real or perceived demand on the body or mind) that triggers the stress response. Stress reactivity is defined as the emotional and physical responses to the demand (Blonna, 2007; Choi, Vickers, \& Tassone, 2014). This terminology is used throughout the current dissertation.

The impact of stress on eating has been well studied. In the general population, many people (including those without psychopathology) modify their eating behaviours in response to stress. This eating change, referred to as stress-induced eating, is typically characterized by an increased consumption of calories and carbohydrate-rich or high-fat foods, even in the absence of hunger and with a lack of homeostatic need for calories (Baumeister, DeWall, Ciarocco, \& Twenge, 2005; Epel, Lapidus, McEwen, \& Brownell, 2001; O'Conner, Jones, Conner, McMillan, \& Ferguson, 2008; Oliver, Huon, Zadro, \& Williams, 2001; Oliver, Wardle, \& Gibson, 2000). A substantial amount of research has been undertaken to examine the correlates and consequences 
of stress-induced eating in both normal and abnormal eaters (i.e., individuals with clinically diagnosed eating disorders). Large individual differences exist in the strength of the drive to eat. Notably, women tend to report significantly more stress-induced eating than men (Greeno \& Wing, 1994; Wansink, Cheney, \& Chan, 2003). Type of stressor, restraint and disinhibition are also factors that differentially impact stress-induced eating in laboratory and real world settings (Heatherton, Herman, \& Polivy, 1991; Wallis \& Hetherington, 2009; Yeomans \& Coughlan, 2009). This dissertation focuses on stress-induced eating as it relates to individuals with BN. Proximal antecedents to the development and maintenance of binge eating in $\mathrm{BN}$ have been a focus of empirical research. Studies have demonstrated that self-reported subjective stress plays a crucial role in precipitating binge eating episodes in individuals with BN (e.g., Crowther, Sanftner, Bonifazi, \& Shepherd, 2001; Davis, Freeman, \& Garner, 1988; Goldschmidt et al., 2014; Smyth et al., 2007). More generally, BN is associated with increased negative perceptions of daily life stress and decreased coping skills (Cattanach \& Rodin, 1988; Crowther \& Chernyk, 1986). These increased perceptions of stress and negative affect precede binges and ultimately contribute to the etiology and maintenance of the disorder (e.g., Cattanach \& Rodin, 1988). Importantly, perceived interpersonal stress has been identified as a specific type of stress that is influential in precipitating binge eating (see Rieger et al., 2010).

The association between stress and binge eating is supported by prominent theories of binge eating development. Two particular theoretical models represent the majority of research in this area. The affect regulation model posits that individuals binge in response to stress or negative mood states in an attempt to reduce anxiety or other negative emotions through the comfort and distraction provided by food (Hawkins \& Clement, 1984; Heatherton \& Baumeister, 1991). The dietary restraint model proposes that individuals who restrict caloric intake to control 
their body weight are particularly susceptible to binge eating when exposed to stress or a negative affective state (Polivy \& Herman, 1985). This model emphasizes the development of a pernicious cycle of dietary restraint, food craving and bingeing, which becomes self-maintaining (Striegel-Moore, Silberstein, \& Rodin, 1986). Theses two models do not compete with one another; rather, they share a number of characteristics. Notably, cognitive and environmental influences are acknowledged within the framework of both models.

The persistence of BN has been most prominently studied within the cognitivebehavioural theory of BN (Fairburn, 1997; Fairburn, Cooper, \& Cooper, 1986), which is influenced by the abovementioned models and has recently been extended to all eating disorders (i.e., the transdiagnostic theory of eating disorders; Fairburn, Cooper, Shafran, 2003). This theory suggests that the overevaluation of weight and shape and their control is central to the maintenance of BN (and all eating disorders). Other clinical features stem directly from this core psychopathology, including: dietary restraint, preoccupation with thoughts about food and eating, repeated checking of body shape and weight or its avoidance, and engaging in extreme methods of weight control. According to the theory, binge eating in $\mathrm{BN}$ is not a direct expression of the core psychopathology; rather, it is largely a result of attempts to adhere to extreme dietary rules. The compensatory behaviour (e.g., self-induced vomiting) follows to mitigate the effects of binge eating on weight, and a powerful self-perpetuating system is activated. Negative affect also plays an important role in maintaining the binge-eating cycle, as it is considered to undermine the ability to maintain strict control over eating (Fairburn, 1997). Another recognized maintaining process involves the extreme self-criticalness that individuals with BN possess, particularly regarding their highly demanding standards in terms of eating, shape and weight and 
their control (Fairburn, 1997; Fairburn, Marcus, \& Wilson, 1993). This results in negative selfevaluation, which leads to an increased desire to achieve control.

In addition to studies of stress response in $\mathrm{BN}$, emerging research is focused on the association between $\mathrm{BN}$ and social anxiety (i.e., individuals with a diagnosis of social anxiety disorder [SAD] or individuals with high self-reported levels of social anxiety symptoms - as a proxy for those with a SAD diagnosis). SAD is characterized by a persistent and exaggerated fear of social or performance situations that may involve scrutiny or judgment by others (APA, 2013). The reason for studying social anxiety specifically in connection to BN is because a number of social anxiety related factors have been posited as commonalities between the two disorders, such as fear of negative evaluation (FNE). Such common factors have been the focus of recent research studies, some of which will be described in paragraphs to follow. These studies have relied heavily on survey methodology; for example, researchers have examined correlations between subclinical symptoms of $\mathrm{BN}$ and trait levels of psychological factors that are elevated in SAD (e.g., FNE; Hinrichsen, Wright, Waller, \& Meyer, 2003). The goal of this work is to further advance understanding of such commonalities, which can ultimately lead to enhanced BN treatment efficacy (Keel, 2013). To effectively achieve this goal, additional work must be completed to better understand the significance of these commonalities (i.e., how social anxiety related factors influence the course of $\mathrm{BN}$ ).

That said, no published study to date has focused on exploring the impact of elevated social anxiety symptoms (and accordingly, the presence of social anxiety related factors) on stress response in $\mathrm{BN}$. As noted above, previous research has demonstrated that stress from interpersonal (social anxiety related) fears play an important role in the provocation of BN symptoms, thus supporting the high rates of comorbidity between BN and SAD. In fact, this idea 
contributed to the foundation of the interpersonal therapy model of BN (Rieger et al., 2010), which helps individuals identify and modify current interpersonal problems that are often associated with social anxiety related fears or irrational beliefs. Rieger and colleagues (2010) noted, however, the paucity of experimental research investigating interpersonal factors in association with eating disorders, including BN. If high levels of social anxiety exaggerate response to stress in individuals with $\mathrm{BN}$, then this particular group of people may be at a greater disadvantage in $\mathrm{BN}$ treatment, particularly that which is group-based, compared to those without high levels of social anxiety. In line with this reasoning, symptoms of SAD in those with BN have been recognized as a barrier for effective help seeking, a cause of decreased engagement in treatment, and a negative prognostic factor for treatment outcome (Goodwin \& Fitzgibbon, 2002). This pattern of findings is particularly significant because SAD is highly comorbid with BN (Godart et al., 2003; Godart, Flament, Lecrubier, \& Jeammet, 2000). Perhaps social anxiety related factors are important contributors to the maintenance of $\mathrm{BN}$. Using an experimental paradigm, one can investigate how response to stress in $\mathrm{BN}$ is influenced by social anxiety and related factors. This research agenda represented the primary objective of this dissertation.

Of course, other psychiatric conditions, in addition to social anxiety, are also highly comorbid with BN and can influence the course of the disorder. Notably, depressed mood or a diagnosis of major depressive disorder (MDD) has received considerable attention in relation to BN psychopathology. The common co-occurrence of MDD and BN is well recognized; in fact, studies indicate that MDD is the most common comorbid diagnosis in individuals with eating disorders, with an estimated lifetime prevalence rate between 50 and $65 \%$ in BN (e.g., Casper, 1998; Hinz \& Williamson, 1987; Hudson, Hiripi, Pope, \& Kessler, 2007). Given this high rate of depressive symptomatology in $\mathrm{BN}$, early researchers had hypothesized that $\mathrm{BN}$ is a mood 
disorder variant rather than a distinct diagnostic entity (e.g., Hinz \& Williamson, 1987; Hudson et al., 1983). The current hypothesis, however, is that BN is often accompanied and fuelled by depressed mood/negative affect, similar to many other psychiatric conditions (e.g., Stice, Bohon, Marti, \& Fischer, 2008; Stice \& Fairburn, 2003). As mentioned earlier in this paper, negative affect is the focus of one major explanatory theory for the etiology and maintenance of BN.

Researchers have questioned whether there are subtypes of $\mathrm{BN}$ that conform to its two major models (negative affect and dietary restraint). If so, distinct risk and maintenance factors might accompany each subtype and inform treatment planning (Stice \& Agras, 1999; Stice \& Fairburn, 2003). Two subtypes of BN have indeed been identified: (1) dietary subtype and (2) dietary-depressive subtype (Stice \& Fairburn, 2003). Individuals characterized by the latter subtype have been shown to experience more severe disturbances including greater bulimic pathology and social impairment, more comorbid psychiatric disorders, and poorer response to cognitive-behavioural treatment (e.g., Grilo, Masheb, \& Berman, 2001; Stice \& Agras, 1999; Stice \& Fairburn, 2003). Based on these findings, the combination of dietary restraint and depressive affect signals a variant of $\mathrm{BN}$ that is of greater severity and more difficult to treat compared to the dietary restraint variant of $\mathrm{BN}$.

While considerable research has investigated the impact of comorbid MDD or depressive symptoms on the course of $\mathrm{BN}$, further research is necessary to investigate the potential impact of other comorbid conditions, namely social anxiety. No known study has directly compared comorbidity rates of MDD or SAD in individuals with $\mathrm{BN}$, but it is important to recognize that both disorders are highly prevalent in $\mathrm{BN}$ and can affect treatment outcome. Given the documented high rate of comorbidity between $\mathrm{BN}$ and SAD (discussed below) there is a need for researchers to investigate the impact of social anxiety symptoms on the course of BN. 
Returning to the notion of stress response, it is important to consider the measurement tool to be used in this investigation. There are various ways to study psychological stress response; commonly used methods involve the induction of stress in a laboratory, or self-reports of stress from one's natural environment. Given that the reliability of retrospective reporting is questionable, ecological momentary assessment (EMA) of stress has gained popularity as an ecologically valid method of assessing naturalistic stress (Keller et al., 2012, Wheeler \& Reis, 1991). A concern, however, with all methods of naturalistic stress investigation (including EMA), is the stress generative effects of psychopathology. This concept refers to the idea that people influence their experience of stress (Vollrath, 2001), and those with psychopathological characteristics tend to interact with the world in a way that generates stressful experiences (Hammen, 1991). Another concern with studying naturalistic stressors is the lack of control over the dose of stress to which a person is exposed. Researchers therefore recognize the benefits of studying stress via an experimental method that relies on laboratory stressors (Allen, Kennedy, Cryan, Dinan, \& Clarke, 2014). With the use of laboratory stressors, researchers control the dose of stress, thereby controlling for stress generative effects.

A variety of laboratory stressors exist to examine the acute stress response; there is a lack of consensus concerning the best stress-induction method (for a review of advantages and disadvantages of distinct stress induction methods, see Allen et al., 2014). The shared goal of these methods is simply to provoke a response, whether the response of interest is physiological (i.e., cardiovascular, endocrine) and/or psychological (i.e., subjective emotional, psychosocial). To date, diverse stress induction methods have been used interchangeably with psychiatric and nonpsychiatric populations to provoke stress in laboratory settings. One stressor alone is typically used in a study, though sometimes two or more stressors are used simultaneously 
without comparing each stressor's unique effects (e.g., Allen, Blascovich, \& Mendes, 2002; Reinhardt, Schmahl, Wust, \& Bohus, 2012); this methodology has occasionally been used in eating disorder literature (e.g., Cattanach, Malley \& Rodin, 1988). Comparing the effects of different stressors is not common practice. One study, however, did compare cortisol responsiveness and alpha-amylase following the Trier Social Stress Test (TSST) and electrical stimulation stress (Maruyama et al., 2012). Results revealed no differences with regard to salivary alpha-amylase response between the two stressors, although the salivary cortisol response to the TSST was significantly increased compared to the salivary cortisol response to electrical stimulation. These results suggest that the hypothalamic-pituitary-adrenal (HPA) axis may show differential response patterns to distinct types of stressors (Maruyama et al., 2012). Unfortunately, Maruyama and colleagues (2012) did not consider psychological or cardiovascular stressor reactivity. It is important to conduct further research on stress response using distinct methods.

Methods of provoking stress across individuals with varied psychological conditions range from those that are physiologically-based, such the carbon dioxide $\left(\mathrm{CO}_{2}\right)$ challenge (Kaye, Buchanan, et al., 2004) and the Cold Pressor Test (Deuter et al., 2012; Schwabe, Haddad, \& Schachinger, 2008), to those that are psychosocially-based, such as the Trier Social Stress Test (TSST; Kirschbaum, Pirke, \& Hellhammer, 1993) and the Mannheim Multicomponent Stress Test (Maruyama et al., 2012). Other stress-provocation methods have been developed for use in specific populations. In eating disorder research, for example, several different methods have been used to provoke stress via effects on body image, including negative weight-related feedback (e.g., Mills \& Miller, 2007), film clips (e.g., Svaldi, Caffier, Blechert, \& TuschenCaffier, 2009) and an ineffectiveness induction (e.g., McFarlane, Urbszat, \& Olmsted, 2011). 
In order to address the overall objective of this dissertation (i.e., examine the effects of different types of stress on those with BN and the potential influence of comorbid psychiatric symptoms), it was important to carefully consider the types of laboratory stressors that were employed. Response to a psychosocial stressor might be affected by social anxiety related symptoms to a significantly greater degree than response to other types of stressors. Increasingly, researchers are questioning the notion that response is consistent across stressors (Dickerson \& Kemeny, 2004; Skoluda et al., 2015). Investigating the differential effects of stressor types on BN symptoms (while taking into account comorbid psychiatric symptoms/conditions) can provide important information about maintenance mechanisms of BN. For this reason, the secondary objective of this dissertation was to make a methodological contribution to the study of stress response (in BN specifically) by comparing reactivity across three distinct laboratory stressors.

Two of these stressors, the TSST and the $\mathrm{CO}_{2}$ challenge, are established laboratory stress provocation methods. The TSST is a 15-minute multicomponent stressor involving three challenging tasks: preparing a presentation, performing the presentation in front of an evaluative audience, and completing a mental arithmetic assignment. Each of these tasks is designed to be stressful by enforcing unrealistic time constraints to meet expected goals (see Kudielka, Hellhammer, \& Kirschbaum, 2007 for a detailed description of the TSST protocol). The TSST is most commonly used in psychosocial stress-induction studies (see Allen et al., 2014 for a review). Exposure to the TSST can lead to different subjective effects, depending upon one's individual appraisal of the stressor (Lazarus and Folkman, 1984); however, stress (and accordingly, the TSST) is generally perceived as a negative experience. For example, the TSST has been shown to increase self-reported stress and anxiety, worsen negative mood, and reduce 
calmness, even in nonclinical, healthy individuals (see Allen et al., 2014). The TSST also reliably induces biological stress, most notably increased HPA axis, sympathetic-adrenalmedullary (SAM), and cardiovascular activation (increased heart rate and systolic blood pressure). The TSST or variations of this test (i.e., variations of the speech and mental arithmetic tasks) has become one of the most popular methods for the experimental induction of acute psychosocial stress (Campbell \& Ehlert, 2012).

Although $\mathrm{CO}_{2}$ challenges have been used for decades to induce panic attacks, the use of this method for physiologically based stress induction is relatively new. This method relies on a single, vital capacity inhalation of $35 \% \mathrm{CO}_{2}$-enriched air, and has become recognized as a valid and reliable method of provoking the acute human stress response (Kaye, Buchanan, et al., 2004). Although the $\mathrm{CO}_{2}$ challenge is considered as a predominantly physiological stressor, it can be argued that a psychosocial component is inherent within it, given the constant interaction with a researcher during the task and the potential of embarrassment while wearing the physiological equipment (e.g., salivating excessively onto the mouthpiece).

In studies of healthy, nonpsychiatric individuals, the $\mathrm{CO}_{2}$ challenge has been shown to produce sympathetic, parasympathetic, and HPA axis activation, coupled with a mild degree of increased subjective anxiety (e.g., Argyropoulos et al., 2002; Kaye, Soothill, Hunt, \& Lightman, 2004; Wetherell et al., 2006). Less research has focused on response to the $\mathrm{CO}_{2}$ stressor in psychiatric populations compared to healthy individuals; this is generally true of all laboratory stressors, not only the $\mathrm{CO}_{2}$ challenge (as an example, see Allen et al., 2014 for an overview of TSST studies in psychiatric populations). The stress research using $\mathrm{CO}_{2}$ is very much in its infancy. In striking contrast, decades of research have used the same testing method accompanied by some of the same outcome measures to study reactivity to the $\mathrm{CO}_{2}$ challenge - 
primarily in panic disorder (PD) populations, but also in other psychiatric and nonpsychiatric populations. Emphasis in these studies, however, has often been on panic attack rates, and possible mechanisms of reactivity have often focused on panic-related variables. That noted, findings from these panic studies could arguably be used to inform stress research; this is discussed below in greater detail.

The third stressor utilized in this study (in addition to the TSST and the $\mathrm{CO}_{2}$ challenge) was one that targets stress uniquely associated with body image and is therefore particularly relevant to individuals with eating disorders. As noted above, some laboratory tasks are designed to elicit stress within specific populations. Those that are used in existing eating disorder research can, in theory, be useful for identifying specific mediators (i.e., body image dissatisfaction) of stress response in eating disorder populations. However, any effects of these stressors as a result of body image are potentially confounded by social factors (e.g., FNE, social comparison) given the social components inherent within these stressors. For this reason, a body image stressor (BIS) was developed for use in this dissertation. This stressor consisted of a slideshow adapted from a film clip used in a previous study that examined binge-eating triggers. The 92-second film clip depicts two women talking to one another while walking around in a fashion store (Svaldi et al., 2009). All explicit social aspects of this clip were removed (two women talking) while retaining its body-related content (articles of clothing) in static images. Doing so would decrease the potential for social comparison and other social anxiety related cognitions as a result of viewing the images. Findings from a study piloting the use of this stressor are described in Appendix A. Overall, pilot testing suggested that the BIS provoked heightened subjective reactivity in a disordered eaters group compared to a healthy eaters group (Linett, Carney, Antony, \& Vickers, 2016). 
To overview, this dissertation had three major objectives, each focused on extending the study of stress response in individuals with BN. The first objective was to compare stressor response in individuals with $\mathrm{BN}$ (BN group) compared to individuals without a history of eating disorders (i.e., a non-eating disorder [NED] group). The second objective was to address the predictive power of social anxiety symptoms and correlates (e.g., FNE) on stressor response within each group. The following research questions were advanced: Do social anxiety symptoms predict reactivity to stressors in the BN group and in the NED group? Do correlates of social anxiety predict response to stressors in people with $\mathrm{BN}$ and in the NED group? In the BN group specifically, are other common comorbid conditions (e.g., depressed mood) associated with stressor response to the same extent as social anxiety symptoms? The third objective was to examine whether distinct laboratory stressors differentially affect individuals with BN, in order to inform researchers about the appropriateness of using these stressors interchangeably to provoke stress in a $\mathrm{BN}$ population.

In the pages to follow, a review of existing literature in two converging research areas (social anxiety symptoms in $\mathrm{BN}$ and stress response) is presented prior to detailing the methodology of the present study.

\section{The Relationship Between BN and SAD}

\section{Comorbidity of BN and SAD: Prevalence rates and temporal sequencing. Studies}

have demonstrated high rates of comorbidity between eating disorders and anxiety disorders overall. For instance, the presence of at least one anxiety disorder was found in $63.5 \%$ of individuals with an eating disorder $(N=672)$ from the Price Foundation Collaborative Genetics Study (Kaye, Bulik, Thornton, Barbarich, \& Masters, 2004). Similarly in two studies of individuals seeking treatment for eating disorders, $71 \%(N=271)$ and $65 \%(N=100)$ of those 
with an eating disorder had at least one anxiety disorder, respectively (Godart et al., 2003; Swinbourne et al., 2012). Several studies have been sufficiently large to subtype those with eating disorders into DSM-IV-defined diagnostic groups. Kaye, Bulik et al. (2004) reported that across the total sample and within diagnostic groups (e.g., anorexia nervosa [AN] and $\mathrm{BN}$ ), obsessive-compulsive disorder was the most common co-occurring disorder (total sample, 41\%), followed by SAD (total sample, 20\%). The prevalence of SAD did not differ significantly across eating disorder diagnostic groups ( $\mathrm{AN}, \mathrm{BN})$, and the rate of $\mathrm{SAD}$ in $\mathrm{BN}$ was $16 \%$. Godart et al. (2003) found that SAD was the most frequent lifetime anxiety disorder diagnosis in those with both BN purging subtype (36\%) and BN nonpurging subtype (36.8\%). Conversely, another study found higher lifetime rates of SAD in individuals with $\mathrm{BN}$ purging (64.7\%) compared to $\mathrm{BN}$ nonpurging (31.1\%; Garfinkel et al., 1996). Notably, DSM-5 no longer subtypes purging and nonpurging behaviours in a $\mathrm{BN}$ diagnosis. Other studies have shown similarly high prevalence rates of SAD in those with $\mathrm{BN}$ (irrespective of subtype), from both community and treatmentseeking samples (Godart, Flament, Perdereau, \& Jeammet, 2002). Notably, these rates are significantly higher than the lifetime prevalence of SAD in the general population, which is approximately $12 \%$ (Ruscio et al., 2008).

Data suggest that $\mathrm{SAD}$ also co-occurs with $\mathrm{AN}$ - both the restrictive and binge-purge subtypes (Deep, Nagy, Weltzin, Rao, \& Kaye, 1995; Godart et al., 2003; Godart et al., 2000; Kaye, Bulik et al., 2004; Swinbourne et al., 2012). In some studies, the rates of SAD in AN are similar to those in BN (Godart et al., 2003), while in other studies they are significantly lower than those in BN (Bulik, Sullivan, Fear, \& Joyce, 1997; Iwasaki, Kriike, Matsunaga, Nagata, \& Yamagami, 1999). This latter finding may reflect a lack of social interactions in AN rather than a 
lack of social anxiety (Pallister \& Waller, 2008). The focus of this dissertation, however, is on BN only; therefore, AN will not be discussed further.

Several studies have also investigated the temporal sequencing of eating and anxiety disorders. These studies have found that, in most cases, the onset of anxiety disorders precedes the onset of eating disorders (Godart et al., 2003; Godart et al., 2000; Kaye, Bulik et al., 2004); therefore, anxiety disorders are posited to be a risk factor for eating disorders (Bulik et al., 1997). This pattern of findings has held true within studies of SAD specifically. For example, SAD age of onset has been found to predate age of onset for $\mathrm{BN}$ among those with both disorders (e.g., Godart et al., 2003, 2000; Schwalberg, Barlow, Alger, \& Howard, 1992). In fact, two studies reported that among individuals diagnosed with both disorders, $90 \%$ experienced the emergence of $\mathrm{SAD}$ before $\mathrm{BN}$, possibly suggesting that $\mathrm{SAD}$ represents a type of predisposing vulnerability to the later development of BN (Brewerton et al., 1995; Godart et al., 2002). In another study, higher levels of social anxiety were found to predict bulimic behaviours over a 7-month period (Gilbert \& Meyer, 2005a). A more recent longitudinal investigation, however, suggested a causal link between $\mathrm{SAD}$ and $\mathrm{BN}$ in the opposite direction than what has been found in the aforementioned studies (Buckner, Silgado, \& Lewinsohn, 2010). Specifically, after controlling for age, sex, major depressive disorder and other anxiety disorders, a diagnosis of $\mathrm{BN}$ in adolescence was found to increase the risk of developing SAD. Additional work is necessary to understand the temporal relations between $\mathrm{SAD}$ and $\mathrm{BN}$. This will not be addressed within the current dissertation, however, which focuses on the cross-sectional relationship between $\mathrm{BN}$ and social anxiety.

Research approaches to studying common factors between BN and SAD. In studying the commonalities between these two disorders, researchers have taken one of two main 
approaches: (1) to examine shared vulnerability factors for the development of both $\mathrm{BN}$ and SAD; or (2) to examine mechanisms shared between these two disorders that contribute to their maintenance. Both approaches aim to enhance understanding of the high comorbidity between $\mathrm{BN}$ and $\mathrm{SAD}$ by suggesting that there are similar psychological mechanisms underlying the symptom expressions of both disorders (Arcelus, Haslam, Farrow, \& Meyer, 2013; Buckner et al., 2010; Levinson \& Rodebaugh, 2012). Both approaches also similarly address the major objective of this study, which is to identify factors (social anxiety related) that contribute to the maintenance of BN. This literature will be briefly reviewed in the section below.

Social anxiety related factors that are common in BN. Through research on factors shared between $\mathrm{BN}$ and $\mathrm{SAD}$, much can be learned about mechanisms that maintain $\mathrm{BN}$. The factors that have received the most attention in studies to date are FNE, social appearance anxiety (SAA) and perfectionism. Importantly, each of these factors is related to social anxiety. FNE (Watson \& Friend, 1969) is defined as social-evaluative anxiety, or more specifically, “apprehension about others' evaluations, distress over their negative evaluations, avoidance of evaluative situations, and the expectation that others would evaluate oneself negatively" (p. 449). FNE is recognized as a core dimension of social anxiety; research supports the notion that social anxiety is derived in part from fears of perceived negative evaluation (e.g., Clark \& Wells, 1995; Rapee \& Heimberg, 1997). SAA is a negative evaluative fear that focuses specifically on one's overall appearance (Hart et al., 2008). Although not always central to one's social anxiety, SAA is indeed recognized as another dimension of the disorder (Moscovitch, 2009). Perfectionism is typically defined as a multidimensional construct (Frost, Marten, Lhart, \& Rosenblate, 1990) that comprises two overarching types: (1) adaptive perfectionism (i.e., striving for high standards), 
which is related to healthy functioning (DiBartolo, Frost, Chang, Lasota, \& Grills, 2004), and (2) maladaptive perfectionism (i.e., critical self-evaluation based on a drive to achieve unrelenting and unrealistic high standards), which is related to negative outcomes such as anxiety and depression (Bieling, Israeli, \& Antony, 2004; DiBartolo \& Frost, 2008). Maladaptive perfectionism has also been implicated as an important factor in the clinical manifestations of social anxiety (Antony, Purdon, Huta, \& Swinson, 1998).

Before discussion of these potential shared mechanisms, it is necessary to provide an overview of the dual pathway model of BN (Stice \& Agras, 1998), because a number of the studies to follow were shaped within the context of this prominent etiological model. The dual pathway model combines aspects of the sociocultural model (Striegel-Moore et al.,1986), the affect regulation model (Hawkins \& Clement, 1984; Heatherton \& Baumeister, 1991), and the dietary restraint model (Polivy \& Herman, 1985). Five risk factors are proposed to be involved in the development of bulimic behaviours: (1) social pressures to be thin; (2) internalization of the thin ideal; (3) body dissatisfaction; (4) dieting; and (5) negative affect. The model theorizes that perceived pressure to be thin from parents, peers and the media, and an internalization of the thin-ideal (i.e., the notion that it is important to attain a thin figure and engage in behaviours to attain this ideal) increase body dissatisfaction because this ideal is extremely difficult to achieve. Increases in body dissatisfaction subsequently increase the risk of dieting as a means of attaining the thin ideal. There is also a subsequent increase in negative affect because of the central evaluative dimension that North American culture places on physical appearance. Dieting and negative affect are thought to mediate the other risk factors in the model and result in bulimic symptoms. This model has been tested through several studies, all of which have found that these five risk factors prospectively predict bulimic psychopathology, with the full model accounting 
for up to $33 \%$ of the variance in the development of bulimic symptoms (Shepherd \& Ricciardelli, 1998; Stice, 2001; Stice, \& Agras, 1998; Stice, Shaw, \& Nemeroff, 1998). It should be pointed out, however, that a large amount of variance remains unaccounted for in this model; there is certainly room for improvement in the prediction of bulimic symptoms, thus leading to an examination of factors that could potentially be added to this model and likewise to maintenance models of BN.

Fear of Negative Evaluation (FNE). FNE has undergone the most extensive study thus far as a factor common to both $\mathrm{BN}$ and SAD. FNE is conceptualized as a cognitive vulnerability or risk factor for social anxiety (Haikal \& Hong, 2010; Rapee \& Heimberg, 1997) as well as a general indicator of social evaluative fear (Clark \& Wells, 1995; Moscovitch, Orr, Rowa, Reimer, \& Antony, 2009). It is typically measured by the brief version of the Fear of Negative Evaluation Scale (Leary, 1983). The eating disorder literature suggests that FNE is related to several established risk factors of the dual pathway model of BN (Stice \& Agras, 1998). In particular, researchers have linked FNE to body dissatisfaction (e.g., Striegel-Moore, Silberstein, \& Rodin, 1993; Vander Wal \& Thomas, 2004). To illustrate, Striegel-Moore and colleagues (1993) found that while women with BN were more concerned with social evaluation compared to those without psychiatric diagnoses, higher levels of FNE were predictive of body dissatisfaction in all women regardless of BN presence. Additionally, in a cross-sectional study of a nonclinical sample of women, FNE predicted drive for thinness over and above depression and the tendency to engage in social comparison, whereas social comparison predicted bulimic symptoms (Gilbert \& Meyer, 2003). In a subsequent longitudinal study by these researchers, FNE did not predict a longitudinal change in restrictive eating attitudes (drive for thinness and body dissatisfaction); rather, it predicted increases in bulimic attitudes, whereas poor self-esteem 
predicted increases in body dissatisfaction (Gilbert \& Meyer, 2005b). An additional investigation revealed that FNE in general relationships (but not close relationships) was uniquely correlated with body dissatisfaction and drive for thinness, but not bulimic symptoms (Gilbert \& Meyer, 2005a). FNE may therefore play a role in the development of body dissatisfaction because it amplifies perceived pressure from acquaintances and society in general (Gilbert \& Meyer, 2005b).

In further studies of clinical participants, converging evidence suggests that elevated FNE is associated with bulimic symptoms in addition to restrictive eating (Hinrichsen et al., 2003). This finding is consistent with Gilbert and Meyers' (2005b) longitudinal study results summarized above, and the idea that bulimic symptoms often develop after restrictive pathology as a means to block awareness of negative emotions associated with FNE (Fairburn \& Harrison, 2003; Heatherton \& Baumeister, 1991; Keel, Mitchell, Miller, Davis, \& Crow, 2000). Indeed, individuals may restrict food intake in the short term, although if this mechanism of dealing with FNE is not sufficient in the long term they may develop bulimic symptoms. Authors of a more recent cross-sectional study investigated the relationships between FNE, the risk factors of the dual pathway model, and bulimic symptoms using path analysis (Utschig, Presnell, Madeley, \& Smits, 2010), finding that FNE was uniquely associated with bulimic symptoms above and beyond the other risk factors in the model. Interestingly, two relationships in the model were no longer significant with the addition of FNE (body dissatisfaction and negative affect, dietary restraint and negative affect); thus, FNE may be a stronger predictor of negative affect compared to body dissatisfaction and dietary restraint (Utschig et al., 2010).

Another recent study examined reciprocal longitudinal relations between FNE and eating disorder risk factors in the context of an ED prevention program for college-aged women 
(Deboer et al., 2013). Findings revealed that FNE predicted subsequent body dissatisfaction and eating disorder (ED) symptoms. FNE also predicted subsequent thin-ideal internalization among women with high body mass index (BMI), but not among those with low BMI. Taken together, findings from this literature suggest that FNE plays an important role in maintaining BN symptoms.

Social Appearance Anxiety (SAA). Given the role of appearance fears in SAD and the emphasis that individuals with eating disorders place on their appearance, SAA is posited to be influential in the relationship between SAD and BN. SAA is recognized as unique construct (Hart et al., 2008); it differs from negative body image, which focuses on a general dissatisfaction in one's self image because of body dissatisfaction. It also differs from social physique anxiety as measured on the Social Physique Anxiety Scale (Hart, Leary, \& Rejeski, 1989), which refers to concerns about one's body form and structure (e.g., body tone, proportions). SAA, on the other hand, encompasses all of one's appearance. It is typically measured by the Social Appearance Anxiety Scale (SAAS; Hart et al., 2008), which has been shown to predict state social anxiety experienced from an appearance evaluation (Hart et al., 2008; Levinson \& Rodebaugh, 2011). SAA has been demonstrated to contribute a unique proportion of variance in social anxiety beyond measures of negative body image, such as the Appearance Schemas Inventory (Cash \& Labarge, 1996) and the Body Image Ideal Questionnaire (Cash \& Szymanski, 1995). It also predicts social anxiety when other constructs are controlled for, such as depression, personality traits and affect (Hart et al., 2008; Levinson \& Rodebaugh, 2011).

In the eating disorder literature, it has been proposed that social evaluation of appearance leads to disordered eating in individuals who place emphasis on their physical appearance 
(Rieger et al., 2010). In support of this proposition, in an undergraduate sample, Levinson and Rodebaugh (2012) found that SAA predicted body dissatisfaction, bulimic symptoms, shape and weight concerns, and eating concerns over and above other domains of social anxiety: fear of scrutiny, social interaction anxiety, and fear of positive evaluation. SAA had a unique relationship with body dissatisfaction and bulimic symptoms, whereas FNE did not in this study; specifically, FNE uniquely predicted drive for thinness and restraint. Structural equation modeling supported SAA and FNE as vulnerabilities for both social anxiety and ED symptoms, and SAA and FNE both mediated the relationship between SA symptoms and disordered eating (Levinson \& Rodebaugh, 2012). In another study using a clinical sample, higher levels of SAA were found in individuals with a diagnosis of BN compared to healthy controls (Koskina, Van den Eynde, Meisel, Campbell, \& Schmidt, 2011). Similarly, Claes et al. (2012) validated the SAAS in a sample of eating disorder patients, and found that SAA was positively related to BMI, drive for thinness, and body dissatisfaction in women diagnosed with an ED (Claes et al., 2012).

Perfectionism. Perfectionism has also received considerable attention as a link between $\mathrm{BN}$ and SAD, especially in a recent review of the perfectionism literature (Egan, Wade, \& Shafran, 2011). Perfectionism is a complex construct; it has been predominantly measured by two Multidimensional Perfectionism Scales, each with distinct factor structures. The 35-item Frost Multidimensional Perfectionism Scale (FMPS; Frost, Marten, Lhart, \& Rosenblate, 1990) measures six dimensions of perfectionism: concern over mistakes, doubts about actions, personal standards, parental expectations, parental criticism, and organization. The 45-item Hewitt and Flett Multidimensional Perfectionism Scale (HMPS; Hewitt, Flett, Turnbull-Donovan, \& Mikail, 1991) measures three dimensions of perfectionism: self-oriented perfectionism, other-oriented perfectionism, and socially prescribed perfectionism. Bieling et al. (2004) performed factor 
analyses using both scales, demonstrating clear overlap between them and testing the importance of distinguishing between the two types of perfectionism (maladaptive, adaptive). A 2-factor solution was produced, consisting of Evaluative Concerns (concern over mistakes, doubts about actions, parental concerns, parental expectations, and social-prescribed perfectionism) and Positive Striving (personal standards, organization, self-oriented perfectionism and otheroriented perfectionism).

Maladaptive perfectionism is posited to contribute to the development and maintenance of social anxiety by eliciting an assumption in socially anxious individuals that social interactions will be negative (embarrassing) because they will be unable to meet a very high standard of social performance (Heimberg, Juster, Hope, \& Mattia, 1995). This idea was supported by high scores in individuals with social anxiety disorder on the CM, DA, and PC scales of the FMPS and socially prescribed perfectionism (i.e., perceiving others as having high expectations) on the HMPS (Antony, Purdon et al., 1998; Juster, Heimberg, \& Holt, 1996; Lundh \& Öst, 1996). Furthermore, some studies examined the role of perfectionism in the cognitive behavioural treatment of social anxiety. Overall perfectionism (total FMPS score; Ashbaugh et al., 2007; Lundh \& Öst, 2001) as well as scores on the CM and DA subscales of the FMPS (Ashbaugh et al., 2007) declined following Cognitive Behavioural Therapy for social anxiety. However, with respect to Ashbaugh and colleagues' study, findings should be interpreted with caution; the degree of change in perfectionism scores were negligible compared to the large effect sizes that were found for social anxiety measures.

Several reviews suggest that maladaptive perfectionism is a risk factor for the development of eating disorders (Bardone-Cone et al., 2007; Jacobi, Hayward, de Zwaan, Kraemer, \& Agras, 2004; Stice, 2002), including BN; for example, perfectionism predicted later 
bulimic symptoms in several prospective studies (Steele, Corsini, \& Wade, 2007; Vohs, Bardone, Joiner, Abramson, \& Heatherton, 1999). Cross-sectionally, HMPS subscales associated with adaptive perfectionism, such as self-oriented perfectionism, were also found to be elevated in BN compared to healthy controls (Castro-Fornieles et al., 2007). Among people with BN, perfectionism is thought to relate to an overemphasis on attractive physical appearance (Brewerton et al., 1995). In one study, women who reported that their bodies do not conform to thin-ideals were more likely to endorse bulimic symptoms (Cash \& Szymanski, 1995).

Perfectionism and social anxiety may also interact to explain significant variance in bulimic symptoms (Silgado, Timpano, Buckner, \& Schmidt, 2010). Shame and avoidance of emotional expression (i.e., not displaying emotions) are positively related to perfectionism (Geller, Cockell, Hewitt, Goldner, \& Flett, 2000; Stoeber, Harris, \& Moon, 2007), and two studies demonstrated that shame (Grabhorn, Stenner, Stangier, \& Kaufhold, 2006) and avoidance of emotional expression (McLean, Miller, \& Hope, 2007) at least partially accounted for the relationship between social anxiety and disordered eating. Thus, perfectionism may have played a role in these relationships. To explore this hypothesis, researchers conducted a study of undergraduate students and found that individuals with high levels of both social anxiety and perfectionism demonstrated the greatest number of bulimic behaviours (Silgado et al., 2010). In this study, high levels of perfectionism were linked with a stronger association between social anxiety and bulimic behaviours (but not drive for thinness or body dissatisfaction). Perfectionism was measured using the Eating Disorder Inventory (EDI) perfectionism subscale, which includes items related to two facets of perfectionism: self-oriented perfectionism (i.e., excessive personal expectations) and socially prescribed perfectionism (e.g., beliefs that others have excessive expectations of them). In support of these findings, one research group using the FMPS found 
that maladaptive perfectionism fully mediated the relationship between fear of public scrutiny and bulimic symptoms and partially mediated the relationship between social interaction anxiety and bulimic symptoms, above and beyond maladaptive body image cognitions and adaptive perfectionism (Menatti, Weeks, Levinson, \& McGowan, 2013).

Investigating FNE, SAA, and perfectionism in the same model. A recent cross-sectional study tested a combined model of risk using an undergraduate sample, in which maladaptive and adaptive perfectionism, FNE, and SAA were included as shared risk factors for social anxiety and eating disorder symptoms (Levinson et al., 2013). SAA was uniquely associated with both social anxiety and eating disorder symptoms on the EDI-2 (Garner, Olmsted, and Polivy, 1983), over and above maladaptive and adaptive perfectionism, FNE, gender, body mass index, trait negative affect, and depression. FNE in this study was uniquely associated only with social anxiety and not eating symptoms; this finding is inconsistent with previous research findings of FNE as a predictor of drive for thinness and bulimic symptoms (Gilbert \& Meyer, 2003, 2005a; Utschig et al., 2010). However, the previous work did not consider SAA; this specific form of FNE may be what drives the relationship between FNE in general and eating disorder symptoms. Neither facet of perfectionism emerged as a risk factor of either set of symptoms; again, the additional constructs measured might explain this study's results. The authors speculate that SAA may drive the relationship between perfectionism and eating disorder symptoms, rather than the perfectionistic standards themselves.

\section{Anxiety sensitivity as another factor common in both SAD and BN. Anxiety}

sensitivity (AS; Reiss, \& McNally, 1985) is an additional potential shared correlate between $\mathrm{BN}$ and SAD that could contribute to stress response in BN. AS, as measured by the Anxiety Sensitivity Index (Reiss, Peterson, Gursky, \& McNally, 1986), is the fear of anxiety related 
sensations based on beliefs that such sensations have negative implications (e.g., illness, embarrassment, or additional anxiety (Reiss \& McNally, 1985). Because of this heightened sensitivity to negative affective states (Otto, Pollack, Fava, Uccello, \& Rosenbaum, 1995; Schmidt, Lerew, \& Jackson, 1997), individuals with elevated AS may be more likely to make behavioural attempts to reduce their negative affect relative to people with low AS. This construct has undergone extensive investigation over the past two decades and has been well established as a risk factor for the development and maintenance of anxiety psychopathology (Reiss, 1991; Taylor, Koch, \& McNally, 1992; Zvolensky et al., 2006).

Several investigations have focused specifically on the link between AS and SAD, demonstrating that heightened levels of AS are significantly associated with a diagnosis of SAD and social anxiety symptoms (Anderson \& Hope, 2009; Cox, Parker, \& Swinson, 1996; Norton et al., 1997); this finding is particularly true for one dimension of AS, the fear of anxiety sensations due to their feared social consequences (Deacon \& Abramowitz, 2006; Rector, Szacun-Shimizu, \& Leybman, 2007; Taylor et al., 2007; Wheaton, Deacon, McGrath, Berman, \& Abramowitz, 2012). The presence of this relationship emphasizes the heightened self-focused attention that is seen in individuals with SAD (Clark \& Wells, 1995; Hofmann, 2007; Rapee \& Heimberg, 1997), which exacerbates cognitive and somatic (interoceptive) symptoms of social anxiety. In other words, individuals with SAD may become fearful of observable physical sensations of anxiety (e.g., blushing, sweating) because of the feared social consequences (i.e., heightened AS social concerns, such as embarrassment), thus further exacerbating their anxious response.

As mentioned above, individuals with high AS have difficulty implementing adaptive affect-regulatory strategies (Kashdan, Zvolensky, \& McLeish, 2008); in individuals with BN, 
bingeing and purging are used as maladaptive means to regulate affect (Heatherton \& Baumeister, 1991). Along those lines, a significant relationship was found between AS and BN symptoms in both undergraduate and outpatient psychiatric samples while controlling for the effects of impulsivity, mood and anxiety symptoms (Anestis, Holm-Denoma, Gordon, Schmidt, \& Joiner, 2008). No other study to date has investigated AS in BN samples, although the investigation of AS in overweight samples as a predictor of obesity is gaining interest (Hearon, Utschig, Smits, Moshier, \& Otto, 2013).

\section{Stress Reactivity in BN}

Experimentally investigating stress response in individuals with $\mathrm{BN}$ provides significant insight into factors that maintain the disorder. Indeed, researchers have used both naturalistic and laboratory stressors to investigate stress response in $\mathrm{BN}$, and results generally suggest that individuals within this diagnostic group experience heightened reactivity to stressors (e.g., Monteleone et al., 2012; Woznica, Vickers, Koerner, \& Fracalanza, 2015). However, no study to date has directly examined psychological and physiological stress reactivity in individuals with $\mathrm{BN}$ while considering the potential effects of comorbid symptoms and disorders, including those that are social anxiety related (and known to be linked to BN, as demonstrated above). The review to follow will contain further details of the stressors to be employed in the proposed study, and the psychological (subjective) and cardiovascular (heart rate, blood pressure) effects of these stressors in BN populations, where applicable. Neuroendocrine effects will not be a focus because these effects (e.g., cortisol) were not tested within this dissertation. The primary reason for this omission was that budgetary restraints prevented this testing. 


\section{The TSST}

The TSST is arguably the most widely utilized psychosocial method of laboratory stress induction. The standardized TSST procedure is as follows (Allen et al., 2014): When participants enter the laboratory, they first undergo an initial rest period to establish a true baseline.

Following this rest period, participants are introduced to a role-playing scenario in which they are told to prepare a speech to convince a panel of assessors that they are the perfect candidates for a job; they must then present this speech to the panel. Next, participants are told to complete a mental arithmetic task involving serial subtraction. A second rest period then commences and poststressor measures are taken. Typically, multiple time points are used to record changes in biological and psychological effects of the TSST; Allen et al. (2014) presents a detailed figure depicting the typical procedure and time points of measurement.

The multiple components of the TSST have been found to reliably produce moderate stress in a majority of participants (Campbell \& Ehlert, 2012). As mentioned earlier in this dissertation, response to the TSST most notably involves increased HPA axis activity (heightened adrenocorticotropic hormone $[\mathrm{ACTH}]$ and cortisol secretion), increased SAM activity (enhanced adrenaline and serum amyloid A), and altered cardiovascular activity (e.g., increased heart rate and systolic blood pressure - and to a lesser extent, diastolic blood pressure). Cortisol and the ACTH are the most frequently studied responses to the TSST; cardiovascular and psychological (subjective) responses are less often reported. Recent research is also investigating effects of the TSST on immune system activity and the brain-gut axis (Allen et al., 2014). Few studies have specifically investigated reactivity to the TSST in SAD and BN populations. 
According to some studies, adults with SAD exposed to a social-evaluative task responded with increased subjective anxiety and increased heart rate (Hofmann, Newman, Ehlers, \& Roth, 1995), along with elevated cortisol reactivity (Roelofs et al., 2009). Other studies replicated increased subjective anxiety in socially anxious individuals or those with SAD compared to controls after this type of stressor, but did not find differences in cardiovascular or cortisol reactivity (e.g., Anderson \& Hope, 2009; Beaton, Schmidt, Ashbaugh, Santesso, \& Antony, 2006; Grossman, Wilhelm, Kawachi, \& Sparrow, 2001). One study found that the subjective experiences of unreality both in one's sense of self (depersonalization) and in the outside world (derealization; Simeon, 2004) were more frequent symptoms in SAD than in controls after the TSST (Hoyer, Braeuer, Crawcour, Klumbies, \& Kirschbaum, 2013). Furthermore, these symptoms were positively correlated with safety behaviours and postevent processing (specifically in relation to the TSST procedure) even after controlling for social anxiety.

One study of individuals with disordered eating (without a formal eating disorder diagnosis) found blunted salivary cortisol, cardiac output, heart rate, and stroke volume in response to laboratory-induced acute psychological stress (using the TSST) compared to the responses of healthy controls (Ginty, Phillips, Higgs, Heaney, \& Carroll, 2012). Systolic and diastolic blood pressure poststressor did not differ between groups. These findings suggest that disordered eating behaviour may be characterized by dysregulation in both the neuroendocrine and sympathetic nervous system branches of the stress response system. However, these results were not supported in another study that found normal cortisol and alpha amylase response to the TSST in individuals with BN (Monteleone et al., 2012). 
Various factors (in addition to stress appraisal) moderate TSST response. Moderating factors include those that are social anxiety related, in line with the social nature of the TSST. Specifically, studies have shown a moderating effect of self-reported social support (Ditzen et al., 2008; Eisenberger, Taylor, Gable, Hilmert, \& Lieberman, 2007; Foley \& Kirschbaum, 2010) and perfectionism, such that a low level of perceived social support and a high level of perfectionism in everyday life are each associated with a greater increase in TSST response although only with respect to cortisol as the outcome (Richardson, Rice, \& Devine, 2014; Wirtz et al., 2007). Psychological (subjective) response to the TSST has not been examined with respect to these moderating factors, but this is recognized as an important area in need of further research (Campbell \& Ehlert, 2012).

High levels of trait anxiety should also be considered with respect to TSST response. State anxiety is defined as a transitory emotional state, whereas trait anxiety is a proneness to experience state anxiety frequently, across time and situations (Gros, Antony, Simms, \& McCabe, 2007). This disposition to experience anxiety has been described as a personality trait (Spielberger, 1966). Individuals with high levels of trait anxiety typically respond to stressors (arguably all stressors, not only the TSST) with greater cognitive and physiological arousal compared to individuals with low levels of trait anxiety (e.g., DeMoja \& DeMoja, 1986; Grillon, Ameli, Foot, \& Davis, 1993). However, other studies have found that high trait anxiety is associated with lower levels of physiological response to acute psychosocial stress despite reports of subjective anxiety (e.g., Jezova, Makatsori, Duncko, Moncek, \& Jakubek, 2004). Not only does this speak to the discordance between physiological and psychological stress responses but also to the concept of allostatic load (Campbell \& Ehlert, 2012), which can explain the 
potential effects of high trait anxiety over time. Allostatic load might also explain Ginty and colleagues' (2012) findings described above.

\section{The $\mathrm{CO}_{2}$ Challenge}

General overview. As mentioned earlier, the $\mathrm{CO}_{2}$ challenge is a physiological provocation that, in those with $\mathrm{PD}$, provokes intense and transitory symptoms of panic rather than a gradual rise in anxiety (although symptoms of anxiety can certainly accompany panic). For decades now, the administration of $\mathrm{CO}_{2}$ enriched-air in controlled laboratory settings has been used to provoke psychological and physiological symptoms of panic and anxiety. Variations exist in the general $\mathrm{CO}_{2}$ paradigm concerning gas concentration and method of administration. Some researchers utilize a continuous inhalation of a low percentage mixture (5$15 \% \mathrm{CO}_{2}$ ) for a period of 15 - 30 minutes, a 20 -second inhalation of either $13 \%$ or $20 \%$, or one or two vital capacity inhalations of $35 \% \mathrm{CO}_{2}$ (Rassovsky \& Kushner, 2003). The physiological process that produces panic and anxiety differs depending on the administration method. For this reason, the results of $\mathrm{CO}_{2}$ studies with different delivery methods are not comparable. Panic from the single or double breath inhalation is produced immediately by acute hypercapnia (respiratory acidosis; higher levels of $\mathrm{CO}_{2}$ in the blood) followed by a hypocapnic overshoot (respiratory alkalosis; a loss of $\mathrm{CO}_{2}$ from the blood) (McNally, 1994).

Practical implications have prompted many researchers to utilize the $35 \% \mathrm{CO}_{2}$ challenge (Griez, Lousberg, van den Hout, \& van der Molen, 1987), involving a single breath inhalation of $35 \% \mathrm{CO}_{2}$ balanced with $65 \%$ oxygen. This method is known to produce the highest level of $\mathrm{CO}_{2}$ exposure, albeit for the shortest time. The procedure is consistent, easily administered, and well tolerated (Vickers, Jafarpour, Mofidi, Rafat, \& Woznica, 2012), and it shows good test-retest consistency across two inhalations (Verburg, Pols, de Leeuw, \& Griez, 1998). Laboratory study 
participants are told that they will inhale a gas through a mask or mouthpiece; this gas will have a concentration of $\mathrm{CO}_{2}$ that is greater than that in normal room air, and thus it may induce shortlived effects ranging from hardly noticeable changes to strong, autonomic or anxiety-like symptoms. Reactivity or response to the challenge is consistently measured by ratings of subjective anxiety on a visual analogue scale (VAS) and panic symptoms on a questionnaire (i.e., a list of DSM panic attack symptoms); examples of commonality used questionnaires include the Panic Symptoms List (Schruers et al., 2000), the Acute Panic Inventory (Liebowitz, Gorman, Fyer, Dillon, \& Klein, 1984), the Diagnostic Symptoms Questionnaire (Sanderson, Rapee, \& Barlow, 1989) and the Panic Symptoms Inventory (Clark \& Hemsley, 1982).

Some procedural aspects of the $35 \% \mathrm{CO}_{2}$ challenge are not consistent across studies. Most notably, some researchers have participants inhale two gaseous mixtures: one of $35 \% \mathrm{CO}_{2}$ and another of normal room air (i.e., a placebo condition). These two mixtures are sometimes counterbalanced across participants to control for order effects, and other times the placebo inhalation occurs first to give participants an opportunity to stop the experiment before the $\mathrm{CO}_{2}$ inhalation that is much more likely to provoke discomfort (e.g., (Argyropoulos et al., 2002; Harrison et al., 1989) Arguably, a placebo condition is not essential to the procedure as participants are able to familiarize themselves with the breathing apparatus during a baseline practice period, thus reducing potential anticipatory anxiety derived from the breathing apparatus.

The $\mathrm{CO}_{2}$ challenge as a panic induction method. The $35 \% \mathrm{CO}_{2}$ challenge incites pronounced anxiety and panic attacks in many persons with PD (Gorman et al., 1990; Griez \& Schruers, 1998; Perna et al., 1994), but not in those with various other psychiatric disorders (e.g., (Griez, de Loof, Pols, Zandbergen, \& Lousberg, 1990; Perna, Barbini, Cocchi, Bertani, \& 
Gasperini, 1995; Verburg, Griez, \& Meijer, 1994; Verburg, Griez, Meijer, \& Pols, 1995). In addition, individuals without a diagnosis of PD but who have elevated genetic risk for PD (e.g., first or second degree biological relatives with PD; (van Beek \& Griez, 2000), a history of unexpected panic attacks (Perna, Gabriele, Caldirola, \& Bellodi, 1995), or dispositional traits known to characterize persons with PD, such as elevated AS (Reiss et al., 1986), show significantly greater reactivity to $\mathrm{CO}_{2}$ than those who do not have enhanced risk. More generally, the $\mathrm{CO}_{2}$ challenge has clear utility in laboratory settings for advancing the understanding of causes and correlates of panic.

The $\mathrm{CO}_{2}$ challenge as a stress induction method. More recently, the $35 \% \mathrm{CO}_{2}$ challenge has also been established as a stress test. In healthy individuals, acute hypercapnia is mildly anxiogenic; those who undergo the $\mathrm{CO}_{2}$ challenge typically report a transient increase in anxiety and various bodily sensations that are characteristic of panic (Griez \& Schruers, 2003). The experience of a complete panic attack is extremely rare. Given this response in healthy individuals, stress researchers proposed a second use of the $\mathrm{CO}_{2}$ challenge: as an acute physiological stressor that mimics the acute human stress response (Argyropoulos et al., 2002; Kaye, Buchanan, et al., 2004). A number of studies have investigated this use of the $\mathrm{CO}_{2}$ challenge, and it has been demonstrated as a well-tolerated and noninvasive method of simultaneously activating psychological and physiological responses to stress (i.e., the key stress response systems). More specifically, the $\mathrm{CO}_{2}$ stress test promotes activation of the parasympathetic, sympathetic, and HPA systems, while decreasing heart rate and increasing systolic blood pressure (Kaye, Buchanan, et al., 2004; Shufflebotham et al., 2009; Wetherell et al., 2006). Importantly, no extinction of these responses occurred at retest 6 weeks later (Kaye, Buchanan, et al., 2004; Wetherell et al., 2006). This lack of extinction is in marked distinction to 
pure psychological stressors, such as the TSST, which usually only provoke stress the first time a participant undergoes the test (Gerra et al., 2001).

Thus far, the $\mathrm{CO}_{2}$ challenge as a stress test has been used to enhance understanding of how stress reactivity relates to health. Researchers have examined how certain conditions (e.g., irritable bowel syndrome, lactation in postpartum women, exam anxiety; Kaye, Soothill et al., 2004; Loft et al., 2007; Shufflebotham et al., 2009) affect stress responding in healthy individuals (without psychiatric illnesses). In these studies, various sensations in addition to anxiety are often measured on a VAS; for anxiety specifically, the average increase from pre- to post- $\mathrm{CO}_{2}$ is usually modest but significant (see Appendix B for a table depicting the sensations measured across studies). A measure of stress appraisal is typically also included within these studies, as cognitive appraisal of a situation is theorized to play a fundamental role in the stress process (Lazarus \& Folkman, 1984).

\section{Integration of the $\mathrm{CO}_{2}$ panic and stress literatures? Researchers have not yet}

administered $35 \% \mathrm{CO}_{2}$ as a stress test to a psychiatric population; nor have they considered the role of individual difference variables (such as AS) in augmenting stress response to $\mathrm{CO}_{2}$. As mentioned earlier, the panic literature, in contrast, has frequently tested psychiatric populations with the $35 \% \mathrm{CO}_{2}$ challenge and offers a wealth of information about dispositional variables that promote reactivity to $\mathrm{CO}_{2}$. Logically, it follows that findings from one literature (e.g., the role of AS in promoting anxious response to $\mathrm{CO}_{2}$ in panic provocation studies) ought to apply to the other literature; the two uses of the $\mathrm{CO}_{2}$ challenge seem to be measuring the same phenomenon (i.e., abnormal [exaggerated] response to $\mathrm{CO}_{2}$ ), yet within different populations. If so, a synthesis of these literatures (which are currently distinct) might make sense (Vickers et al., 2012). Importantly, $\mathrm{CO}_{2}$ response is often assessed in both literatures by ratings on a visual analogue 
scale (VAS) and a panic symptom scale. The only difference is that one additional outcome is often assessed within the panic literature, namely whether a panic attack has occurred according to defined criteria (involving increases in anxiety and panic symptoms). While the synthesis of these two literatures is not an objective of the current dissertation, the review below adopts the perspective that researchers can learn about $\mathrm{CO}_{2}$-stress response in specific psychiatric groups from the $\mathrm{CO}_{2}$-panic literature.

How do people with $\mathrm{SAD}$ respond to $35 \% \mathrm{CO}_{2}$ ? Several studies have investigated the response to $\mathrm{CO}_{2}$ in people with SAD compared to people with $\mathrm{PD}$ and healthy controls. The interest in this comparison arose from a hypothesis that the pathogenesis of SAD and PD may be similar (Gorman et al., 1990), and thus a similar response to $\mathrm{CO}_{2}$ would be expected in these two groups. Due to methodological differences (and therefore an inability to compare results) across studies using dissimilar $\mathrm{CO}_{2}$ percentages (Blechert, Wilhelm, Meuret, Wilhelm, \& Roth, 2010; Holt \& Andrews, 1989a, 1989b; Rapee, Brown, Antony, \& Barlow, 1992), only studies using $35 \% \mathrm{CO}_{2}$ are considered here.

Regrettably, data are scarce in terms of physiological response to $\mathrm{CO}_{2}$ in those with SAD. Gorman et al. (1990) and Papp et al. (1993) both found that $\mathrm{CO}_{2}$ produced comparable increases in heart rate, diastolic pressure, and systolic blood pressure across a healthy control group and a SAD group. Other $\mathrm{CO}_{2}$ studies of SAD participants either did not employ physiological measures or such results were not reported in research articles. On the other hand, more studies have reported on psychological $\mathrm{CO}_{2}$ reactivity in $\mathrm{SAD}$; these studies have yielded inconsistent findings. Some suggested that individuals with SAD reacted similarly to healthy controls (Gorman et al., 1990; Schutters, Viechtbauer, Knuts, Griez, \& Schruers, 2012), while others found similar reactivity in SAD and PD (i.e., significantly greater than that in healthy controls; 
(Caldirola, Perna, Arancio, Bertani, \& Bellodi, 1997). Moreover, another study found intermediate $\mathrm{CO}_{2}$ reactivity in $\mathrm{SAD}$, suggesting that individuals with $\mathrm{SAD}$ are significantly less reactive than those with PD but more reactive than healthy controls (Papp et al., 1993). Effect size estimates of these comparisons, with VAS ratings of anxiety as the outcome variable, can be found in Appendix C.

A meta-analysis of the abovementioned studies was conducted by Schutters et al. (2012), in terms of three $\mathrm{CO}_{2}$ response indicators: panic attacks, subjective anxiety, and panic symptomatology. Findings of this meta-analysis demonstrated a similar pattern of results for all three $\mathrm{CO}_{2}$ response indicators, such that $\mathrm{CO}_{2}$ reactivity was greatest in the $\mathrm{PD}$ group, followed by the SAD group and then the healthy control ( $\mathrm{HC}$ ) group. Moreover, $\mathrm{CO}_{2}$-elicited panic attack rates in SAD participants were significantly stronger than those of HCs but significantly weaker than those of people with PD (Schutters et al., 2012). Increases in subjective anxiety scores post$\mathrm{CO}_{2}$ were significantly higher in the PD group compared to the $\mathrm{HC}$ group, but the SAD group did not differ significantly from those with PD or from HCs. In terms of panic symptoms, differences between groups were not significant. In summary, based on this meta-analysis, it appears that the experience of a panic attack post- $\mathrm{CO}_{2}$ may be specific to individuals with $\mathrm{PD}$, whereas subjective anxiety and panic symptoms may increase post- $\mathrm{CO}_{2}$ in a similar manner across individuals with PD or SAD.

The abovementioned studies did not consider cognitive characteristics that predispose individuals to the development of panic psychopathology and thus may account for the relationship between $\mathrm{SAD}$ and $\mathrm{CO}_{2}$ reactivity. It was speculated that degree of $\mathrm{CO}_{2}$ reactivity might be a function of vulnerability to (threatening) interoceptive cues (i.e., AS; Schutters et al., 2012; Schmidt \& Richey, 2008). As mentioned earlier in this dissertation, AS is significantly 
related to $\mathrm{SAD}$-specific cognitions; therefore, $\mathrm{CO}_{2}$-induced symptoms (e.g., sweating, trembling, shortness of breath) may incite fear due to perceptions of these symptoms as catastrophic, embarrassing, or signs of weakness/incompetence -- and thus potential targets for evaluative judgment (Anderson \& Hope, 2009; Spector, Pecknold, \& Libman, 2003). Accordingly, another study investigated whether social anxiety symptoms were uniquely associated with $\mathrm{CO}_{2}$ reactivity by controlling for the presence of specific cognitive variables (AS and negative affect; (Schmidt \& Richey, 2008). In a nonclinical undergraduate sample without a history of panic attacks, findings supported both high levels of AS and social anxiety symptoms (measured by total score on the Liebowitz Social Anxiety Scale; Liebowitz, 1987) as predictors of subjective anxiety and panic symptoms post- $\mathrm{CO}_{2}$. Total score on this scale emerged as a unique predictor of $\mathrm{CO}_{2}$ response; however, a later evaluation of its six subscales failed to reveal more specific mechanisms (e.g., performance fears, observational fears) linking $\mathrm{SAD}$ to $\mathrm{CO}_{2}$ reactivity. Nonetheless, it is possible that specific $\mathrm{SAD}$ fears might also contribute to the exaggerated $\mathrm{CO}_{2}$ response in individuals with SAD.

How do people with $\mathrm{BN}$ respond to $35 \% \mathrm{CO}_{2}$ ? In comparison to $\mathrm{SAD}$, the study of $\mathrm{CO}_{2}$ reactivity in persons with eating disorders has received far less attention. One study found comparable rates of reactivity (i.e., subjective anxiety and panic symptoms) in an eating disordered group (AN and BN) and a HC group (Perna et al., 2004); panic attack rates were not measured. However, limitations of this heterogeneous eating disordered group prompted researchers to conduct another $\mathrm{CO}_{2}$ challenge test in a group of participants with $\mathrm{BN}$ only (Woznica et al., 2015). Contrary to Perna et al.’s findings, reactivity in the BN group was significantly greater than that in the $\mathrm{HC}$ group; and furthermore, reactivity was comparable in the BN group and a PD group (see Appendix C for effect sizes). Specifically, change in subjective 
anxiety ratings from pre to post- $\mathrm{CO}_{2}$ was comparable in the $\mathrm{BN}$ group $(M=11.04, S D=14.61)$ and the PD group $(M=7.18, S D=28.24)$, and both groups' changes in anxiety were greater than that of the HC group $(M=-10.32, S D=25.23)$. A similar pattern was seen with panic symptoms, such that change from pre to post- $\mathrm{CO}_{2}$ was comparable in the $\mathrm{BN}$ group $(M=2.36$, $S D=1.98)$ and the PD group $(M=2.27, S D=3.95)$, and both groups' changes in panic symptoms were greater than those of the HC group $(M=-0.07, S D=2.00)$.

Although not discussed in the published manuscript (Woznica et al., 2015), several predictors of reactivity were considered in that study: state anxiety, trait anxiety, depressive symptoms, AS and distress tolerance (Woznica, Vickers, Koerner, \& Fracalanza, 2014). Across the entire sample, only AS was significantly correlated with subjective anxiety and panic symptoms. Within group analyses demonstrated a significant correlation only between AS and subjective anxiety in the PD group; however, these analyses lacked power due to small group sample sizes and thus conclusions cannot be made. Another important limitation of this study was that social anxiety symptoms were not measured and therefore were not considered in analyses as a potential mechanism of reactivity. Moreover, cardiovascular effects of $\mathrm{CO}_{2}$ reactivity in $\mathrm{BN}$ were not assessed and indeed have not been measured in any studies to date. Future studies are needed to extend the study of $\mathrm{CO}_{2}$ reactivity in $\mathrm{BN}$, and with a larger sample size, as was employed in the current study, unique predictors of reactivity within groups were considered.

Mechanisms of $\mathrm{CO}_{2}$ Reactivity. Within the panic literature, a slew of studies have examined potential cognitive mechanisms of $\mathrm{CO}_{2}$-reactivity. The mechanism that has most consistently emerged across studies is AS. That is, high AS has been implicated as a significant predictor of $\mathrm{CO}_{2}$-reactivity in individuals with PD (Perna, Romano, Caldirola, Cucchi, \& 
Bellodi, 2003; Schmidt et al., 1997), in nonpsychiatric samples (e.g., Eifert, Zvolensky, Sorrell, Hopko, \& Lejuez, 1999), and in psychiatric groups aside from PD, including SAD (Schmidt \& Richey, 2008) and BN (Woznica et al., 2015).

State anxiety (and sometimes trait anxiety) are often assessed and considered as two other potential important mechanisms of $\mathrm{CO}_{2}$-reactivity. Studies have failed to consistently find that state or trait anxiety affect $\mathrm{CO}_{2}$-reactivity (for a review, see Zvolensky \& Eifert, 2001), even in studies of individuals with both eating disorders and high anxiety levels (Perna et al., 2004; Woznica et al., 2015). Notably, in a recent study of individuals with PD, a gender-specific relation was found between both state and trait anxiety and $\mathrm{CO}_{2}$ reactivity; measured by the State-Trait Anxiety Inventory (Spielberger, 1983), these factors predicted heightened $\mathrm{CO}_{2}$ induced panic only in females (Monkul et al., 2010). Despite concerns regarding the validity of the this measure (for example, an inability of the trait scale to adequately discriminate between symptoms of anxiety and depression; Gros et al., 2007), the measure continues to be widely used and thus Monkul and colleagues' (2010) findings should be carefully considered.

\section{Eating Disorder Specific Stressor}

\section{Overview of existing tasks that provoke stress in eating disordered populations. As}

mentioned earlier in this dissertation, various tasks have been used to provoke a stressful response in clinical eating disordered populations and nonclinical populations with abnormal eating attitudes or behaviours. An exhaustive review of such tasks is beyond the scope of this dissertation; however, several examples below will depict why these types of tasks were not appropriate for use in current study (i.e., given the social component[s] inherent within them). For instance, some studies have investigated the effects of negative weight-related feedback from others on body image disturbance and disordered eating (e.g., McFarlane et al., 2011; Mills \& 
Miller, 2007; Stice, 2002). According to Baumeister (1997), a threat to self-esteem such as receiving an unfavourable evaluation on a dimension previously judged as important to one's self-concept (e.g., body weight) decreases self-esteem and increases negative affect. One such study of negative weight-related feedback found that female restrained eaters, but not unrestrained eaters, reported feeling depressed, anxious, and worse about their appearance after being weighed on a scale that was rigged to report a weight five pounds heavier than their actual weight (McFarlane et al., 2011). This finding was supported in another study, which also suggested that a peer's perception of weight is more important than that of a nonpeer (Mills \& Miller, 2007). Evidently, social comparison might play a role in these results.

In another study examining binge eating triggers, a film clip was used that depicts two women speaking to each other while walking around in a fashion store for 92 seconds (Svaldi et al., 2009). The film clip elicited a significant increase in anxiety, sadness, and desire to binge in participants with binge eating disorder (BED) compared to control participants without BED (Svaldi et al., 2009). The clip was selected based on its ability to elicit low intensity and neutral valence ratings in control participants without an eating disorder (Hewig et al., 2005); thus, it is possible that that the body-related content was the major emotion eliciting factor in the eating disorder group (Svaldi et al., 2009). However, the potential for social comparison related factors to influence emotion after viewing the film clip is obvious given that two women were depicted interacting in a social situation.

\section{The Present Study}

Given the abovementioned research, it was appropriate to conduct an experimental study of stress response in $\mathrm{BN}$ and the potential influence of social anxiety and related factors. This study is novel and important for several reasons. It is one of the first studies of stress response in 
BN to consider whether social anxiety (or other psychological conditions such as depression) predicts stress response in this population. Consequently, this study can contribute to the growing body of research on $\mathrm{BN}$ and social anxiety by investigating whether high social anxiety predicts especially marked response to stress in BN. This research is important because it could potentially suggest an explanation for poorer prognosis in this subset of individuals with BN. Ultimately, this knowledge could inform the treatment of $\mathrm{BN}$ through the promotion of clinical research studies to investigate the efficacy of social anxiety treatment components within a BN treatment protocol. In addition, this study was the first to utilize the $\mathrm{CO}_{2}$ stress test (recently noted as an ideal stressor; Kaye et al., 2004) as a stress-induction method within the eating disorders literature. This study therefore queries the expanded use of this physiological stressor, which could be of great benefit to laboratory stress research. Moreover, this was the first study to compare reactivity to three stressors within BN. Generally in stress research, the effects of distinct stressors on a particular population are not compared; yet it is important to remember that all stressors should not necessarily be considered equal. This study can therefore make an important methodological contribution to the study of stress response in a $\mathrm{BN}$ population.

Objectives. The first major objective of this study was to investigate whether individuals with BN respond more strongly to stressors compared to those without a history of eating disorders. This was accomplished by the following investigations. First, subjective (self-reported, emotional) and physiological (cardiovascular: heart rate, blood pressure) responses to three distinct laboratory stressors (a modified TSST, the $\mathrm{CO}_{2}$ challenge, and a body image stressor) at time 1 (prestressor) and time 2 (poststressor) were compared within groups. Subsequently, change in responses from time 1 to time 2 was compared between individuals with $\mathrm{BN}$ and individuals without a history of eating disorders. Several factors that could have potentially 
influenced stress response (given previous research) were assessed to support the contention that a BN diagnosis itself predicts greater response to stress. These factors were as follows: depressive symptoms (measured by the depression subscale of the Depression Anxiety Stress Scales - 21 item version; DASS-21-D), state and trait anxiety (measured by the state and trait versions of the State Trait Inventory for Cognitive and Somatic Anxiety; STICSA), anxiety sensitivity (measured by the Anxiety Sensitivity Index - 3; ASI-3), stress appraisal (measured by the Perceived Stress Scale; PSS), and stressful life events (measured by the Holmes-Rahe Stress Inventory; HRSI).

Mechanisms that predict response to each stressor within each group were then investigated. The factors examined were those that have been considered in previous research linking BN with social anxiety and related factors (fear of negative evaluation, social appearance anxiety, perfectionism). Fear of negative evaluation was measured by the Brief Fear of Negative Evaluation Scale (BFNE), and social appearance anxiety was measured by the Social Appearance Anxiety Scale (SAAS). Three aspects of perfectionism were evaluated: maladaptive perfectionism (measured by a combination of subscales of the HMPS and FMPS; Bieling et al., 2004), self-oriented perfectionism (measured by a subscale of the HMPS), and worry about perfectionism (measured by a subscale of the Physical Appearance Perfectionism Scale; PAPS). In addition, within the $\mathrm{BN}$ group alone, whether other common comorbid symptoms (depression, trait anxiety) were associated with stressor response to the same extent as social anxiety symptoms was examined. Also within the BN group alone, the possible link between stress reactivity (anxiety, fear, stress, and panic symptoms) and eating/body image related response (body dissatisfaction, desire to eat, hunger) was evaluated. 
The last objective was to examine whether the three distinct laboratory stressors differentially affected individuals with BN. In other words, this study addressed whether participants with $\mathrm{BN}$ respond to each stressor with different levels of anxiety, fear, stress, panic symptoms, body dissatisfaction, desire to eat, and hunger, and what factors (social anxiety related, if any) predict differential response to each stressor. This was accomplished through within-subjects comparisons (in the $\mathrm{BN}$ group only) of subjective and physiological responses to the three laboratory stressors.

Hypotheses. In line with each of the abovementioned study objectives, the following hypotheses were advanced.

1.1. In response to each stressor, participants in the BN group would report significantly greater increases in VAS ratings of anxiety, fear, and stress, and PSL scores compared to participants in the NED group. Participants in the BN group would also report significantly greater VAS ratings of body dissatisfaction, desire to eat and hunger compared to participants in the NED group.

1.2. The magnitude of VAS ratings of anxiety, fear, stress, and PSL scores in response to each of the three stressors would differ between groups. The greatest difference between groups in the magnitude of these stressor responses will be with the BIS.

1.3. Physiological stress responses (heart rate [HR], systolic blood pressure [SYS-BP], diastolic blood pressure [DIA-BP]) would mirror the self-reported stress responses in 1.1 and 1.2; that is, in response to each stressor, participants in the $\mathrm{BN}$ group would demonstrate significantly greater changes in HR, SYS-BP and DIA-BP compared to participants in the NED group. However, as reported earlier in this paper, the divergence between physiological and psychological responses to stressors is well recognized. 
1.4. The magnitude of HR, SYS-BP, and DIA-BP responses would also differ between groups and will be most pronounced with the BIS.

1.5. Participants in the BN group would respond more strongly to each stressor compared to the NED group even when the following variables were taken into account: DASS-21

Depression subscale (DASS-21-D) scores, STICSA State and Trait scores, ASI-3 scores, HRSI scores, and PSS scores.

2.1. TSST and $\mathrm{CO}_{2}$ - In each group, Social Phobia Inventory (SPIN) scores would predict increases in VAS ratings of anxiety, fear and stress, increases in PSL scores, and changes in HR, SYS-BP, and DIA-BP poststressor. These predictions were made based on previous studies examining how individuals with social anxiety respond to each of these stressors. In addition, these relationships would be stronger in the BN group compared to the NED group, and the strongest relationship would be between the TSST and stress response in the BN group. These predictions were made based on the high rates of comorbidity between $\mathrm{BN}$ and social anxiety, and given that the TSST is a psychosocial stressor. In both groups, social anxiety related factors (FNE, SAA, maladaptive evaluative concerns [MEC], self-oriented perfectionism [SOP], worry about imperfection [WAI]) would predict increases in anxiety, fear and stress ratings, PSL scores, and changes in HR and BR poststressor. These relationships would be stronger in the BN group compared to the NED group, and the strongest relationship would be between the TSST and stress response in the BN group.

2.2. BIS - In either group, SPIN scores would not predict increases in VAS ratings of anxiety, fear or stress, increases in PSL scores, or changes in HR, SYS-BP, or DIA-BP poststressor. This is hypothesized because the potential for social comparison has been eliminated from this stressor task. In either group, social anxiety related factors (as stated in hypothesis 2.1.) 
would not predict increases in anxiety, fear or stress ratings, PSL scores, or changes in HR, SYS-BP or DIA-BP poststressor.

3. Compared to SPIN scores, DASS-21-D and STICSA-Trait scores would each have a weaker association with all stress response indicators: VAS ratings (anxiety, fear, stress, body dissatisfaction, desire to eat, hunger), PSL scores, and physiological measures (HR, SYS-BP, DIA-BP).

4. In response to the TSST and BIS, participants with BN would report changes in VAS ratings of anxiety, fear and stress that positively correlate with body dissatisfaction, desire to binge and hunger. No a priori prediction was advanced regarding these associations in response to the $\mathrm{CO}_{2}$ stressor; this was examined in an exploratory fashion.

5. No a-priori hypotheses were advanced regarding the differential stressor effects within $\mathrm{BN}$ participants given the lack of existing data to support directional hypotheses. These associations were investigated in an exploratory fashion. 


\section{Method}

\section{Participants}

A total of 182 individuals indicated an interest to participate in the study, and 137 completed the telephone screening assessment in which eligibility criteria were assessed for two participant groups. Inclusion criteria for a BN group consisted of females with a current principal diagnosis of BN, according to DSM-5 diagnostic criteria. Inclusion criteria for a non-eating disorder (NED) control group consisted of females without a history of the following Feeding and Eating Disorders, according to DSM-5 diagnostic criteria: BN, AN, BED, Other Specified Feeding or Eating Disorder (OSFED), or Unspecified Feeding or Eating Disorder (UFED). Exclusion criteria for all participants were as follows: (1) current/past psychotic or manic episode, any substance use disorder within the past 3 months; (2) clinically significant suicidal ideation; (3) current/past diagnosis of panic disorder; (4) current engagement in psychological treatment, or initiation/completion of psychological treatment within the past 6 months; (5) use of psychotropic medications or medications that could affect heart rate (e.g., beta-blockers), with the exception of benzodiazepines used less than three times a week (and not within 5 half-lives of the study visit); (6) medical conditions that may negatively interact with the transient physiological effects associated with breathing more $\mathrm{CO}_{2}$ than usual (See Appendix D for a full list of medical exclusions); (7) recent participation in another research study that involved a $\mathrm{CO}_{2}$ challenge. Although criterions 3,5,6, and 7 are requirements specific to the $\mathrm{CO}_{2}$ stressor task, they constituted exclusion criteria for the entire study given hypotheses that required withinsubjects comparisons of all three stressor tasks. In addition to the abovementioned eligibility criteria, all participants were asked to agree to the following requests: to abstain from caffeine 
consumption and cigarette use for approximately 4 hours prior to their lab visit, and to abstain from eating for at least 1 hour prior to their lab visit.

Of the 137 individuals who completed the telephone screening assessment, 54 were deemed ineligible to complete the study. Ineligibility was due to the following reasons: endorsement of medical exclusionary criteria $(n=24)$, endorsement of disordered eating symptoms that did not meet criteria for a diagnosis of $\mathrm{BN}(n=15)$, current use of psychotropic medication $(n=12)$, endorsement of an exclusionary psychiatric diagnosis $(n=2)$, and recent participation in another $\mathrm{CO}_{2}$ study $(n=1)$. Eighty-three individuals appeared eligible and were invited to the Psychophysiology Research Laboratory at Ryerson University to complete the study. Of these, 17 individuals either cancelled or did not present for their study appointment. One additional individual presented for her study appointment but did not complete the study due to simultaneous participation in another study of stress response that required her to wear electrodes. Therefore, 65 participants completed the study.

Of these 65 participants, nine were not considered in statistical analyses, as their reported psychological symptoms during the in-person screening procedure were not consistent with diagnostic inclusion criteria for the two study groups. Specifically, three participants in the BN group did not endorse DSM-5 criteria for a current diagnosis of $\mathrm{BN}$ on a diagnostic interview (the MINI International Neuropsychiatric Interview; MINI 7.0) that occurred during the study session. Of these, two participants denied the presence of compensatory behaviours, and one endorsed a low frequency of binge eating and fasting (despite their contradictory report during the telephone screen). One additional participant in the $\mathrm{BN}$ group endorsed diagnostic criteria for BN during the MINI 7.0, but her scores of zero on two eating disorder questionnaires (the Eating Attitudes Test [EAT-26] and the EDI) were inconsistent with her self-report, reflecting a lack of 
disordered eating symptoms. In the HC group, two participants endorsed current disordered eating symptoms during the MINI 7.0 (objective binge eating, fasting and dieting), and another endorsed a history of bingeing and purging despite the absence of these behaviours over the past 8 months. Also in the HC group, one participant scored within the clinical range on the EDI-BN subscale, and one did not complete the measures of subjective stressor response due to softwarerelated technical issues. Accordingly, the final sample size was 56, which consisted of 28 participants in the BN group and 28 participants in the NED group.

Participants were all members of the Greater Toronto Area community. They were recruited from the following sources: (1) flyers posted around the University of Toronto, York University, and Ryerson University ( $n=32)$; (2) advertisements on Craigslist.com and Kijiji.ca (online classifieds; $n=21$ ); and (3) flyers posted on public message boards (e.g., physicians’ offices, community centers; $n=3$ ). Each participant was compensated $\$ 30$ cash for her participation.

Participants ranged in age from 18 to 61 years $(M=26.41, S D=9.94)$. The greatest number of participants reported their ethnocultural background as White (32.1\%), followed by East Asian (23.2\%), East Indian (10.7\%), South Asian (8.9\%), Black (7.1\%), Latin American/Hispanic (5.4\%), Arab or West Asian (3.6\%), and Aboriginal (1.8\%). Three participants $(5.4 \%)$ reported their ethnicity as mixed, and one participant (1.8\%) preferred not to state their ethnicity. participant $(1.8 \%)$ preferred not to state their ethnicity. Canadian-born participants constituted $55.4 \%$ of the total sample. In terms of marital status, $77 \%$ endorsed being single, and $92.9 \%$ denied having any children. Every participant had achieved a high school diploma or equivalent, while $60.7 \%$ of participants completed postsecondary education. Of the 42 participants who reported their annual income, the mean was $\$ 60,785(S D=38,172)$. 


\section{Measures}

Screening measures. The following measures were used to assess study eligibility for study participation.

Health Screening Questionnaire. This questionnaire was developed as a screening tool for use with $\mathrm{CO}_{2}$ studies conducted in Dr. Kristin Vickers' lab (and approved by Ryerson University's Research Ethics Board [REB]). It is a modified, shortened version of the Medical History Questionnaire (described below). Potential participants are asked to respond yes, no, or not sure to questions about their history of various medical conditions. A response of yes or not sure to any question excluded the individual from study participation. This questionnaire was administered over the telephone as part of the screening assessment.

Medical History Questionnaire (MHQ). Dr. Vickers adapted this questionnaire from that used by other $\mathrm{CO}_{2}$ researchers in the United States. It has also been approved by Ryerson University's REB for Dr. Vickers' ongoing lab projects. The MHQ was administered during inperson screening to confirm the absence of medical conditions that would prohibit participation in the $\mathrm{CO}_{2}$ challenge. This was done for two reasons: (1) as a precautionary measure (e.g., an additional medical check required by Ryerson University's REB), and (2) to collect the full range of information provided by the questionnaire (not only information that is related to exclusion criteria, as listed within the Health Screening Questionnaire).

Mini International Neuropsychiatric Interview 7.0 (Sheehan, 2014). The MINI 7.0 is a brief, semistructured diagnostic interview designed to assess current and lifetime DSM-5 (APA, 2013) psychiatric disorders. In this study, select sections were first administered during the telephone screen to assess for a current/past psychotic or manic episode, current substance dependence, current suicidal ideation, current/past panic disorder and eating disorders. The MINI 
7.0 was then administered in its entirety during in-person screening to confirm diagnostic inclusion/exclusion criteria and to assess for the presence of other psychopathology, for the purpose of characterizing the participant sample. This widely used diagnostic interview was chosen because of its reasonable length (approximately 20 minutes) and acceptable psychometric properties in previous versions of the MINI (Sheehan et al., 1998), which were comparable to those of the Structured Clinical Interview for DSM-IV Disorders (First, Spitzer, Gibbon, \& Williams, 2001). For BN, the main diagnosis of interest in this study, kappa was found to be .78 (Sheehan et al., 1998). While psychometric properties specific to the MINI 7.0 are not yet publicly available, the only change in the diagnostic criteria for BN from DSM-IV to DSM-5 is the frequency of binges and purges (i.e., a decrease from twice per week to once per week).

Demographic measure. This measure was constructed specifically for use in this study. It contained basic demographic questions (e.g., age, sex, ethnicity, marital status) that served to characterize the participant sample.

Symptom measures. All participants completed the following standardized self-report questionnaires.

Eating Disorder Inventory (Garner et al., 1983). The EDI consists of 64 questions used to assess pathological eating, cognitions, and behaviours that are common in both AN and BN. Participants are asked to rate each item on a scale ranging from 1 (never) to 6 (always). The EDI yields eight subscale scores; higher scores are indicative of greater eating pathology. In the current study, three specific EDI subscales were considered in analyses: Drive for Thinness (7 items), Body Dissatisfaction (9 items), and Bulimia (7 items). The Drive for Thinness subscale assesses excessive concern with dieting, preoccupation with weight, and fear of gaining weight; the Body Dissatisfaction subscale assesses dissatisfaction with overall body shape and size of 
specific body regions (e.g., hips, stomach, thighs); the Bulimia subscale assesses bulimic behaviours such as binging and purging (Garner et al., 1983). These three subscales assess major vulnerabilities for $\mathrm{BN}$ (and $\mathrm{AN}$ ), and thus they were used to characterize the participant groups and corroborate eating disorder diagnostic status in the BN group. In a BN sample, the internal consistency reliability was found to be high within each of these three subscale (ranging from $\alpha$ $=.83-.92)($ Schaefer, Maclennan, Yaholnitsky-Smith, Stover, 1998). Although two subsequent versions of the EDI have been developed, use of the original EDI in the present study was warranted given the subscales of interest. In the current study's BN group, $\alpha=.66$ for Drive for Thinness, $\alpha=.88$ for Body Dissatisfaction, and $\alpha=.81$ for Bulimia.

Eating Attitudes Test-26-item version (Garner, Olmsted, Bohr, \& Garfinkel, 1982). The EAT-26 is an abbreviated, 26-item version of the EAT-40 (Garner \& Garfinkel, 1979). The EAT-40 was developed as a self-report measure of AN symptoms that was often used as a screening instrument to detect abnormal eating concerns. The EAT-26 was found to be highly correlated with the EAT-40 ( $r=.98$; Garner et al., 1982), and therefore, it is more commonly used in research studies. The EAT-26 has been demonstrated as a reliable and valid measure in both clinical and nonclinical samples. A cut off score of 20 discriminates between disordered and nondisordered eating (Gleaves, Pearson, Ambwani, \& Morey, 2014). Internal consistency reliability was found to be .90 in an AN group, and .83 in a female comparison group (Garner et al., 1982). In the present study, $\alpha=.87$ for the BN group, and .60 for the NED group.

Social Phobia Inventory (Connor et al., 2000). The SPIN is a 17-item self-report measure of social anxiety symptoms. It is designed to assess three symptom dimensions: fear, avoidance, and physiological arousal. Participants are asked to rate the degree to which social anxiety problems have bothered them over the past week. Each item is measured on a 5-point Likert 
scale ranging from 0 (not at all) to 4 (extremely). The SPIN has strong internal consistency in SAD samples (alphas ranging from .87 - .94 for the total scale; Antony, Coons, McCabe, Ashbaugh, \& Swinson, 2006; Connor et al., 2000), control samples of healthy volunteers and psychiatric patients (alphas ranging from $.82-.90$ for the total scale; Connor et al., 2000). In the current study, the SPIN was used as the primary measure of participants' social anxiety symptom levels. Internal consistency reliability of this measure was $\alpha=.93$ for the $\mathrm{BN}$ group, and $\alpha=.93$ for the NED group.

Depression Anxiety Stress Scales - 21-item version (Lovibond \& Lovibond, 1995). The DASS-21 is a self-report measure of three emotional states: depression, anxiety and stress. Each emotional state is assessed on a 7-item subscale, comprising a total scale of 21 -items. The DASS-21 is a shortened version of the DASS-42 (Lovibond \& Lovibond, 1995); DASS-21 scores are doubled and thus comparable to DASS-42 scores. In the current study, only the depression subscale was used for primary analyses. This subscale assesses symptoms of dysphoric mood (e.g., hopelessness) and has strong internal consistency ( $\alpha=0.94$; Antony, Bieling, Cox, Enns, \& Swinson, 1998). This subscale has also demonstrated high convergent validity with other commonly used measures of depression, such as the BDI ( $r=0.79$; Antony, Bieling et al., 1998). The internal consistency of the DASS-21-D in the current sample was $\alpha=$ .89 for the BN group, and $\alpha=.75$ for the NED group.

State-Trait Inventory for Cognitive and Somatic Anxiety - State and Trait Scales (Ree, French, MacLeod, \& Locke, 2008). The STICSA was designed to assess state and trait anxiety, and to improve upon several limitations of the State-Trait Anxiety Inventory (Spielberger, 1983), (e.g., an inability to adequately discriminate between symptoms of anxiety and depression). The STICSA replicates the State-Trait Anxiety Inventory's format of independent state and trait 
scales. They each contain the same 21 self-reported items; such as, my breathing is fast and shallow. The state scale assesses how respondents "feel right now, at this very moment, even if it is not how you usually feel"; the trait scale assesses how respondents "feel in general" (Ree et al., 2008). Responses are rated on a 4-point Likert scale, ranging from 1 (not at all) to 4 (very much so); higher scores reflect greater anxiety. Internal consistency was $\alpha=.92$ for the state scale and .91 for the trait scale (Ree et al., 2008); similar alphas were found in a college sample (Gros et al., 2007). Convergent validity was demonstrated by strong correlations between the STICSA and other state and trait anxiety measures; discriminant validity was demonstrated by weak correlations between the STICSA and depressive symptoms (Gros et al., 2007). Both the state and trait scales were used in the current study. For the trait scale, internal consistency reliability was $\alpha=.89$ for the BN group and $\alpha=.88$ for the NED group. For the state scale, internal consistency reliability was $\alpha=.91$ for the BN group, and $\alpha=.88$ for the NED group.

The Perceived Stress Scale (Cohen, Kamarck, \& Mermelstein, 1983). The PSS is a selfreport questionnaire assessing the degree to which situations in one's life are appraised as stressful, over the past month (Cohen et al., 1983). The PSS was originally developed with 14 items; a shorter 10-item version was subsequently developed that retains the psychometric quality of the original scale (Cohen \& Williamson, 1988). Items are rated on a 5-point Likert scale, from 0 (never) to 4 (very often); higher scores correspond to greater perceived life stress (sample item: how often have you felt that you were unable to control the important things in your life?). The internal consistency of this scale was found to be $\alpha=.78$ (Cohen \& Williamson, 1988). In the current study, this scale was used to measure the level of stress associated with participants' personal lives to ensure that responses to the stress induction tasks are not merely a 
function of PSS scores. The internal consistency of the PSS in the current study sample was $\alpha=$ .87 for the BN group, and $\alpha=.90$ for the NED group.

The Holmes-Rahe Stress Inventory (Holmes \& Rahe, 1967). The HRSI was originally termed The Social Readjustment Rating Scale and later became known as the HRSI. A list of 43 life events is presented to respondents, each of which necessitates a change or adjustment (e.g., changes in residence, in-law troubles, pregnancy, death of a spouse). In comparison to other questionnaires that measure life events, this one is unique in that it not only provides a number and types of events, but also an estimate of the magnitude of these events (see Holmes \& Rahe, 1967 for the method used to assign weights to life events). A total score of $\leq 150$ is indicative of a low susceptibility to stress-induced illness. This scale was used in the current study to determine whether responses to the stress induction tasks are a function of the magnitude of participants' life events.

Anxiety Sensitivity Index - 3 (ASI-3; Taylor et al., 2007). The ASI-3 is an 18-item selfreport measure that assesses fear of bodily sensations associated with arousal. This type of fear differs from state or trait anxiety (McNally, 1994), and is believed to amplify preexisting anxiety and place an individual at an increased risk for panic attacks. The ASI-3 was developed to improve upon the original 16-item version (Reiss et al., 1986) and the ASI-Revised (Deacon, Abramowitz, Woods, \& Tolin, 2003). Three lower-order factors comprise the ASI-3: physical, cognitive, and social concerns (sample item: When I cannot keep my mind on a task, I worry that I might be going crazy). This factor structure has been validated in clinical and nonclinical samples, and has stronger psychometric properties over the original ASI. Each item is rated on a 5-point scale ranging from 0 (very little) to 4 (very much). Higher scores reflect higher levels of anxiety sensitivity. The ASI-3 is a widely used measure, and has demonstrated subscale 
coefficient alphas ranging from $.79-.86$ for physical concerns, $.79-.91$ for cognitive concerns, and .73 - .86 for social concerns (Taylor et al., 2007). In the current study sample, total ASI-3 score was used for analyses; $\alpha=.91$ for the BN group, and $\alpha=.94$ for the NED group.

Brief Fear of Negative Evaluation Scale (Leary, 1983). The BFNE is a 12-item version of the original Fear of Negative Evaluation scale (Watson \& Friend, 1969). It was developed to assess apprehension about negative evaluation from others (sample items: I am afraid others will not approve of me; I am usually worried about what kind of impression I am making on someone). Items are rated on a 5-point scale from 1 (not at all characteristic of me) to 5 (extremely characteristic of me). The BFNE correlates highly with the original FNE scale $(r=$ .96; Collins, Westra, Dozois, \& Stewart, 2005), and also correlates positively with other measures of social anxiety. Psychometric studies demonstrated high internal consistency of the BFNE in college samples $(\alpha=.90)$ and clinical samples $(\alpha=.97)$. Test-retest reliability coefficients were also high in both college samples over a 4-week interval ( $r=.75$; Leary, 1983) and clinical samples over a 2 -week period ( $r=.94$; Collins et al., 2005). In the current study sample, internal consistency of the BFNE was $\alpha=.92$ for the BN group, and $\alpha=.90$ for the NED group.

Social Appearance Anxiety Scale (Hart et al., 2008). The SAAS is a 16-item measure used to assess anxiety about being negatively evaluated by others because of overall appearance, including body shape (e.g., I am concerned people will not like me because of the way I look; I am afraid that people find me unattractive). The SAAS demonstrated high test-retest reliability and good validity indices in college age samples (Hart et al., 2008; Levinson \& Rodebaugh, 2011). Internal consistency was excellent ( $\alpha=.95$; Levinson $\&$ Rodebaugh, 2011). In the current 
study sample, internal consistency of the SAAS was $\alpha=.97$ for the BN group, and $\alpha=.90$ for the NED group.

Frost Multidimensional Perfectionism Scale (Frost et al., 1990) and the Hewitt and Flett Multidimensional Perfectionism Scale (Hewitt, 1991). The FMPS consists of 35 items that measure six dimensions of perfectionism, while the HMPS consists of 45 items that measure three dimensions of perfectionism (described earlier in this paper). Both scales are widely used in research and have been established as valid and reliable measures of perfectionism (Frost, Heimberg, Holt, Mattia, \& Neubauer, 1993; Frost et al., 1990; Hewitt et al., 1991). In previous research, a combination of dimensions from one or both scales has been used to create measures of adaptive and maladaptive perfectionism (Bieling et al., 2004; DiBartolo et al., 2004; Frost et al., 1993). For the current study, two measures of perfectionism were calculated using FMPS and HMPS subscales: 1) maladaptive evaluative concerns, according to Bieling et al. (2004); and 2) self-oriented perfectionism. Internal consistency of the HMPS self-oriented perfectionism subscale within the current study sample was .91 for the BN group, and .91 for the NED group. Internal consistency of the FMPS subscales that comprise the maladaptive evaluative concerns (i.e., maladaptive perfectionism) factor were as follows: concern over mistakes (BN group $\alpha=$ .72 , NED group $\alpha=.85$ ), doubts about actions (BN group $\alpha=.71$, NED group $\alpha=.75$ ), parental concerns (BN group $\alpha=.82$, NED group $\alpha=.80$ ), parental expectations (BN group $\alpha=.79$, NED group $\alpha=.77$ ), and socially-prescribed perfectionism (BN group $\alpha=.84$, NED group $\alpha=.90$ ).

Physical Appearance Perfectionism Scale (Yang \& Stoeber, 2012). The PAPS is a relatively new 14-item self-report measure of perfectionism that focuses on the positive and negative aspects of physical appearance perfectionism. Factor analysis supports the measure's two subscales: worry about imperfection and hope for perfection. Item examples are: I hope that 
my body shape is perfect, I am never happy with my appearance no matter how I dress. The worry about imperfection subscale was used in the current study as an additional measure of perfectionism, one that is focused on physical appearance. Convergent validity of this subscale was demonstrated via positive correlations with negative body image and behaviours to control body weight. Internal consistency was found to be $\alpha=.85$ (Yang \& Stoeber, 2012), and in the current study sample $\alpha=.88$ for the BN group and $\alpha=.84$ for the NED group.

Dependent variables. Two self-report measures were used to assess subjective (psychological) reactivity to stressors. All participants completed these measures immediately before and immediately after each stressor task. Participants were specifically instructed to reflect on their experience of various sensations during the peak of each stressor (i.e., what was the highest level of $X$ you experienced during the task?).

Visual Analogue Scales. VAS are used to measure the degree of subjective intensity of a current sensation on a continuum ranging from 0 (none at all) to 100 (the worst imaginable). VAS ratings of anxiety have been established as the gold standard measure of subjective reactivity to laboratory stressors (Argyropoulos et al., 2002; Kaye, Buchanan, et al., 2004). In the current study, participants were asked to rate the following sensations before and after each stressor: anxiety, fear, stress, body dissatisfaction, desire to eat, and hunger. As noted above, participants were specifically instructed to reflect on their experience of these sensations during the peak of the stressor's effect (the peak is characterized as a subjective experience).

Panic Symptoms List (Schruers et al., 2000). The PSL is a self-report measure that assesses each of the 13 symptoms of physical and cognitive arousal associated with panic attacks. Participants are asked to rate the severity of each symptom from 0 (not at all) to 4 (very severe), with higher scores indicating greater levels of panic symptomatology. Schruers et al. 
(2000) was the first to use the PSL as an outcome measure in $\mathrm{CO}_{2}$-panic provocation studies; this particular measure (or others that similarly assess physical and cognitive arousal) have been used across subsequent $\mathrm{CO}_{2}$ stress studies (e.g., Colasanti et al., 2008). The PSL served as another informative indicator of response to each stressor employed in the current study.

Physiological measures of stress response. HR and BP were measured to assess objective, physiological reactivity to $\mathrm{CO}_{2}$. The BIOPAC MP150 data acquisition system and Noninvasive Blood Pressure Monitoring System, NIBP100D, was used to obtain these cardiovascular measures throughout the completion of each stressor task (from baseline to recovery). Specifically, the researcher placed a double finger cuff on two adjacent fingers of the participant, through which BP (systolic, diastolic) and HR signals were measured and displayed on a blood pressure monitor. An arm cuff was also placed on the participant; it inflated once when the monitoring system was activated, and subsequently when the system required recalibration. Output of a continuous blood pressure waveform was exported directly to a computer through AcqKnowledge 4.2 Software.

Stress-induction tasks. Participants each underwent three laboratory stress-induction tasks: an interpersonal stressor (a modified version of the TSST), a physiological stressor $\left(\mathrm{CO}_{2}\right.$ challenge), and an eating disorder specific stressor (BIS, developed for the present study). The order of these tasks was counterbalanced across participants to control for order effects. Participants within each of the two study groups were randomly assigned to an order, resulting in $5-6$ participants per order in each group.

TSST. A modified version of the TSST was used as an interpersonal stressor (as used in a previous study of BN and stress; Monteleone et al., 2012). The TSST consists of an anticipatory stress period and a task period (Kirschbaum et al., 1993). The TSST procedure in the present 
study was as follows. During the anticipatory stress period, the researcher explained to the participant that she had 5 minutes to prepare a speech for a job application to a committee member, following which time she would have 5 minutes to deliver the speech. She was also informed that her performance would be recorded on a video camera to enable the interview and her nonverbal behaviour to be analyzed later. The anticipatory stress period was followed by the task period, during which time the participant delivered her speech to a confederate. If the participant finished her speech in less than 5 minutes, the confederate urged her to continue speaking until the 5-minute period concluded (using standardized prompts). Immediately after the speech, the participant was asked to perform a mental arithmetic task that required her to serially subtract the number three from 1237 as fast and as accurately as possible within 5 minutes. If a mistake was made, she was asked to start again from 1237. When this 5-minute period elapsed, the participant was told that this portion of the study had concluded, and the confederate left the testing room.

BIS. A slideshow of static images signifying body image stress was selected as a stressor that would particularly affect individuals with eating disorders and associated body dissatisfaction and desire to be thin. The slideshow was adapted from a film clip used in a previous study that examined binge eating triggers, which depicts two women walking around in a fashion store for 92 seconds (Svaldi et al., 2009). In that study, the film clip elicited a significant increase in anxiety, sadness, and desire to binge in those with BED compared to those without BED (Svaldi et al., 2009). The clip was selected based on its ability to elicit low intensity and neutral valence ratings in control participants without an eating disorder (Hewig et al., 2005); thus it was reasonable to hypothesize that the only emotion-eliciting factor in an 
eating disorder group would be the body image related content (and not strong negative affect, for example; Svaldi et al., 2009).

In the current study, this clip was modified to remove its explicit social aspects (i.e., two women talking to each other) while retaining its body image related content (i.e., clothing in a fashion store). Therefore, static images were presented, rather than a film clip with sound and moving images, to decrease the potential for social comparison and other social anxiety related cognitions. Participants viewed the slideshow on a computer monitor while sitting alone in a room. Instructions first appeared on the monitor for 60 seconds: We will now be showing you a short slideshow that contains photographs of clothing articles. Imagine yourself wearing these clothes. It is important for us that you focus on the entire duration of the slideshow. However, if you choose to stop your voluntary participation for any reason, please inform the researcher.

Following these instructions, nine successive images were displayed on the monitor, each for a 10 second duration (see Appendix A for more information about the development and pilot testing of this stressor).

$\boldsymbol{C O}_{2}$. The $\mathrm{CO}_{2}$ challenge was used as a physiological stressor. The following paragraphs provide a description of the $\mathrm{CO}_{2}$ breathing apparatus, a detailed outline of the procedure, and the safety protocol.

Breathing apparatus. The breathing circuit used for the $\mathrm{CO}_{2}$ challenge consists of a disposable $30 \mathrm{~mm}$ ID (inner diameter) mouthpiece (single-participant use) connected to a bacterial/viral filter (Pulmoguard; single-participant use) that connects to a respiratory flowhead, also called a pneumotach, which is a device that measures tidal volume. The pneumotach connects to a two-way nonrebreathing valve, one side of which is exclusively expiration, the other side of which is exclusively inspiration. The inspiratory port connects to a manual 4-way 
stopcock ( 2500 series) with two ports: one port feeds room air, and the second port connects to a gasbag (Hans Rudolph nondiffusing gas collection bag [15 liters]) filled with carbon dioxideenriched air $\left(35 \% \mathrm{CO}_{2}\right.$ balanced with $65 \% \mathrm{O}_{2}$; used exclusively for the $\mathrm{CO}_{2}$-enriched air inhalation).

$\mathrm{CO}_{2}$ Challenge Protocol. The process of the $\mathrm{CO}_{2}$ challenge occurred as follows: In preparation for a participant's arrival to the lab, the investigator disconnected the gasbag from the breathing circuit and filled it with $\mathrm{CO}_{2}$-enriched air from the gas tank. The investigator then reconnected the circuit and attached a new filter to the tubing connected to the gas-mixing chamber, and a new mouthpiece to the other end of the filter. As part of the informed consent procedure, participants were told that they would be inhaling a harmless gas mixture containing $\mathrm{CO}_{2}$ and $\mathrm{O}_{2}$, which might cause transitory discomfort or panic-like symptoms.

When the participant was ready to commence the challenge, she was first connected to the cardiovascular monitoring equipment. In addition to the double finger cuff placed on two adjacent fingers, a finger clip was also placed on the participant's index finger of the same hand to measure oxygen saturation. This measure was recorded by AD Instruments, PowerLab System 8/30 with Chart Pro Modules and displayed on a computer monitor (observed by the researcher). The participant was informed that these measures were acquired continuously throughout the challenge both as a safety precaution to ensure normal cardiovascular function, and for analysis to address study hypotheses.

The participant was then given instructions to connect to the breathing circuit. She was asked to occlude her nose with a nose clip (single-participant use) and insert the mouthpiece into her mouth. She was asked to breathe normally (stopcock feeding room air) for 5 minutes to familiarize herself with the device and reduce potential anxiety that may arise initially from 
procedural aspects of the experiment (i.e., the unnatural experience of breathing through a tube).

During this time, the researcher measured the participant's baseline inspired vital capacity by asking her to inhale as big a breath of room air as possible and hold this breath for 4 seconds before exhaling fully (recorded by AD Instruments, PowerLab System 8/30, with Chart Pro Modules). This vital capacity measure was used as a comparison for the $\mathrm{CO}_{2}$ inhalation; specifically, a participant's $\mathrm{CO}_{2}$ inhalation was considered valid only if it was at least $80 \%$ of her vital capacity.

The $\mathrm{CO}_{2}$ inhalation then occurred. The instructions given for this inhalation were identical to those given for the vital capacity measure. Prior to the participant inhaling deeply, the researcher turned the stopcock to $\mathrm{CO}_{2}$-enriched air; as the participant exhaled, the researcher turned the stopcock back to room-air. The participant then disconnected from the cardiovascular monitoring system and relaxed in a comfortable chair.

Safety Protocol During the $\mathrm{CO}_{2}$ challenge. The researcher was in the same room as the participant throughout the session. The testing session was to end immediately if any of the following conditions occur: (1) if the participant wished to stop at any point; (2) if the participant's SYS-BP reached 170 or above; (3) if the participant's DIA-BP reached 110 or above; (4) if the participant's SYS-BP decreased to 90 or below; (5) if the participant's DIA-BP decreased to 60 or below; (6) if the participant's SYS-BP had a fall of $20 \mathrm{mmHg}$ or more in a 1minute period or in a 3-minute period; or (7) if the participant's DIA-BP had a fall of $10 \mathrm{mmHg}$ or more in a 1-minute period or in a 3-minute period. These blood pressure limits were a precautionary check; in the unlikely event that a participant responds unusually intensely (in terms of blood pressure change), the experiment would stop (and appropriate compensation 
awarded). No participants in the current study experienced any adverse events that required the testing session to end early.

\section{Procedure}

Telephone screening. Females who expressed an interest in completing the study by responding to the advertisements/flyers were invited to undergo a telephone screening assessment to determine eligibility for participation. Following obtainment of verbal consent to participate, there were two components to this screening: (1) questions to assess for lifetime history of specific medical conditions (via the Health Screening Questionnaire, as described above under Screening Measures); and (2) questions to assess for symptoms of select psychiatric disorders within the DSM-5 (via sections of the MINI 7.0, a brief semistructured diagnostic interview). When exclusion criteria were endorsed on the Health Screening Questionnaire, a potential participant was informed that she is not eligible for the study. When a potential participant responded no to all exclusionary items on the Health Screening Questionnaire, then diagnostic screening questions for the various DSM-5 disorders that constituted eligibility criteria (as listed above under Participants) were asked. When a potential participant did not meet any exclusion criteria based on the telephone screening assessment, the researcher invited her to schedule a laboratory visit to complete the study.

Lab visit. All participants completed the study in one, 3-hour individual session. They were first prompted to read and subsequently sign a statement of informed consent, which outlined the general purpose and procedure of the study and potential risks. Participants had an opportunity to ask the researcher questions and, if they wished, to choose not to commence their participation. They were also informed that they can stop their participation at any point in time without penalty, and appropriate course credit/monetary compensation would still be awarded. 
All participants chose to sign the consent form and continue. They were then asked to complete a paper copy of the MHQ. The absence of medical exclusion criteria was confirmed for all participants at that time. This portion of the study took approximately 20 minutes to complete.

Following this, participants were provided with information regarding the cardiovascular monitoring equipment, and they were connected to the cardiovascular monitoring system via a double finger cuff and an arm cuff. Prior to turning on the monitoring system, the outcome questionnaires (VAS, PSL) were described to participants and they were asked to complete the first prestressor measures of subjective response. The monitoring system was then initiated and prestressor measures of HR and BP measurements were recorded. Participants then completed one of the three stressor tasks, as described above, followed by completion of poststressor measures of subjective response, a 5-minute recovery period, and subsequent disconnection from the monitoring system which signaled the completion of stressor task \#1.

Following stressor task \#1, the complete MINI 7.0 (semistructured diagnostic interview) was conducted to determine the presence/absence of mood, anxiety, psychotic, substance use, and eating disorders. This portion of the study took approximately 30 minutes to complete. Participants then completed stressor task \#2, via the same procedure described in the previous paragraph. Following stressor task \#2, participants completed a number of self-report questionnaires (symptom measures) on a computer through Qualtrics, an online survey software program. This portion of the study took approximately 30 to 45 minutes to complete. Stressor task \#3 was then initiated, also via the same procedure described in the previous paragraph. The study concluded with verbal debriefing about the study purpose and procedures. 


\section{Results}

\section{Data Cleaning}

Self-report data. Subjective, self-report data were downloaded from Qualtrics, a web based survey software, into SPSS Statistics. Data were first screened for missing values. It was determined that $0.529 \%$ of all values within the entire data matrix were missing; moreover, missing values did not constitute greater than $10 \%$ of any single variable (i.e., individual questionnaire item), and a total of $28.71 \%$ of variables had at least one missing value. It was also determined that $44.26 \%$ of participants were missing at least one value. An examination of the missing values pattern of the data revealed that missing values in this data matrix were consistent with missing-at-random data. With this careful examination completed, it was considered appropriate to replace the missing values via prorating. Specifically, a participant's responses for all items in a particular scale (or subscale within a scale) were averaged, and this mean was substituted for the missing value. In addition, multiple imputation (Enders \& Gottschall, 2011) was used to impute missing values from all other available information in the dataset. Results from analyses using these two methods were nearly identical, and therefore this dissertation proceeded with the prorated values.

Across all participants, no outliers were found in distributions of the following scale scores: STICSA; DASS-21-D; ASI-3; BFNE; SAAS; PAPS; FMPS; PSS. One outlier was found at the high end of the distribution of HRSI scores; one outlier was found at the low end of the distribution of HMPS scores; and two outliers were found at the high end of the distribution of SPIN scores. Examination of these outliers revealed that there was no pattern among them; each value was derived from a different individual, and could therefore be attributed to random variability across participants. For this reason, all values were retained. A number of additional 
outliers were found in the distributions of VAS and PSL residual change scores. By visual inspection of boxplots and values in the dataset (to check for errors in data input or scoring), it was determined that these outliers were also due to variability in the measurement and clustering of some scores around the zero mark given the inherent nature of these measures. Therefore, they were not excluded from analyses. Within each of the two study groups, skew values for most variables were between -1 and -.05 or between 0.5 and 1 , indicating moderately skewed distributions. One variable (VAS-hunger) within the NED group had a skew value of -2.11, indicating a greater degree of skewedness. Kurtosis values ranged between .500 and 4.16 in the BN group, and 1.55 and 3.89 in the NED group. Values of both skew and kurtosis were not extreme, and analyses proceeded with methods that were robust to these variations of normality (Lorenzen \& Anderson, 1993). The final sample of psychological, self-report data remained at $n$ $=56$.

Physiological data. Physiological data (pulse, SYS-BP and DIA-BP) were examined via AcqKnowledge 4.2 Software. First, a visual inspection of the waveforms within each participant's data file was performed to identify marked artifacts (inaccurate data, resulting from the technology used or experimental error) and determine if the data were interpretable. Several participants' data (BN: $n=7$, NED: $n=5$ ) were removed at this point from further consideration, for the following reasons: difficulty calibrating the $\operatorname{BIOPAC}(n=8)$, experimenter failure to use BIOPAC markers that are necessary to distinguish between time points of the study procedure $(n=3)$, and reported discomfort with the equipment $(n=1)$. The remaining participants $(n=44)$ had complete data files that were subsequently prepared for analysis of physiological data. 
By visual inspection of the data, it was determined that artifacts in the 44 remaining data files could be characterized as noise spikes that were mostly due to physical movement of the participant or poor signal quality from the BIOPAC. These spikes were generally quantified as being greater than three standard deviations (SD) away from the mean. The connect endpoints function was used to remove artifacts within SYS-BP and DIA-BP waveforms. This method preserves the time series of data on the horizontal axis by connecting the edges of the selected area. With respect to participants' HR data, a digital filter (finite impulse response [FIR] band pass filter) was first applied to the raw pulse waveform. This is a necessary step to filter the pulse signal to derive a more accurate calculation of HR (i.e., beats per minute; BPM). An FIR is a linear phase filter, which means that there is no phase distortion between the original signal and filtered waveforms. A band pass filter allows only the data within a specified range to pass through the filter, and thus it preserves the waveform activity. A cutoff frequency for the FIR band pass filter was specified; specifically, a low threshold of 0.5 and a high threshold of 3.0 was applied to define the band of data (the frequency range) that was passed. The number of coefficients was set as four times the sampling rate divided by the cutoff frequency of the filter. Following the application of this filter, the Rate Calculation function was used with a window of 40 to 120 BPM on the filtered waveform. Finally, the connect endpoints function was applied to the rate data (i.e., BPM) to eliminate any remaining artifacts that constituted noise spikes.

Normality of the data was then examined. It was verified that all dependent variables in subsequent analyses (HR, SYS-BP, DIA-BP) approximated the normal distribution. Outliers in the data were also examined carefully within each group. Five outliers were identified in the BN sample, only within the $\mathrm{CO}_{2}$ stressor portion of the physiological recording (not across all stressors). As these outliers were not in the extreme range (i.e., they were between 1.5 and 3 SDs 
away from the mean) they were retained, and thus the final sample of physiological data remained at $n=44$.

Tidal volume data. With respect to the $\mathrm{CO}_{2}$ stressor, the tidal volume of participants' baseline (vital capacity) and $\mathrm{CO}_{2}$ inhalations were measured. These data, acquired by $\mathrm{AD}$ Instruments, PowerLab System 8/30 with ChartPro Modules, were inspected to determine whether the tidal volume of each participant's $\mathrm{CO}_{2}$ inhalation was at least $80 \%$ of her baseline inspired vital capacity. Tidal volume is defined as the quantity of air moved into and out of the lungs during a normal breath. Vital capacity is the maximum amount of air a person can expel from the lungs after a maximum inhalation. Visual inspection of these measurements (in liters) revealed that the tidal volume of each participant's $\mathrm{CO}_{2}$ inhalation was at least $80 \%$ of her vital capacity, and therefore valid for analysis.

\section{Demographics and Psychological Characteristics of the Sample}

Demographics. Table 1 depicts demographic characteristics of the sample, separated into the two study groups. No significant differences between groups were found. Specifically, $t$-tests were not significant for age, $t(54)=0.95, p=.344, r_{\mathrm{pb}}=.13$, annual income, $t(40)=1.33, p=$ $.192, r_{\mathrm{pb}}=.21$, or BMI, $t(54)=-0.65, p=.520, r_{\mathrm{pb}}=.09$. Chi-squares were not significant for ethnicity, $\chi^{2}(10)=8.72, p=.559, \phi_{c}=.394$, marital status $\chi^{2}(6)=5.41, p=.492, \phi_{c}=.311$, number of children, $\chi^{2}(3)=4.31, p=.230, \phi_{c}=.277$, and education level $\chi^{2}(1)=0.30, p=.584$, $\phi=.073$.

Psychological diagnoses. All participants in the BN group met criteria for a current DSM-5 diagnosis of BN. These participants reported a mean of $2.36(S D=1.87)$ objective binges per week over the past 3 months, and $2.96(S D=2.99)$ compensatory behaviours per week over the past 3 months. Of these 28 participants, 22 (78.6\%) reported purging behaviours 
(vomiting, laxative use) and six (21.4\%) reported nonpurging behaviours (fasting, excessive exercise) as compensatory mechanisms. No participants in the NED group met DSM-5 diagnostic criteria for BN, AN, BED, OSFED, or UFED. Participants in both the BN and NED groups completed the EAT-26 and the EDI as screening tools to ensure the presence/absence of disordered eating attitudes or behaviours (means and standard deviations of EAT-26 and EDI scores, separated by group, are displayed in Table 2).

Independent samples $t$-tests revealed that total EAT-26 scores were significantly different between the two groups, $t(32.08)=9.59, p<.001, r_{\mathrm{pb}}=.79$, with the $\mathrm{BN}$ group endorsing significantly greater scores on the EAT-26 compared to the NED group. A standard cutoff score of >19 indicates high risk for an eating disorder (Garner et al., 1982); no participant in the NED group scored above this cutoff. With respect to the EDI, attention was paid to scores on the three subscales that represent measures of disordered eating: Bulimia, Drive for Thinness, and Body Dissatisfaction (Garner et al., 1983). Independent samples $t$-tests, with adjusted degrees of freedom for unequal variances, revealed significantly greater scores in the BN group compared to the NED group on all three subscales: Bulimia, $t(39.58)=11.07, p<.001, r_{\mathrm{pb}}=.79$, Drive for Thinness, $t(32.08)=9.59, p<.001, r_{\mathrm{pb}}=.83$, and Body Dissatisfaction, $t(48.04)=7.49, p<.001$, $r_{\mathrm{pb}}=.71$.

All participants were assessed for other psychological disorders in addition to $\mathrm{BN}$ via the MINI 7.0, as per the DSM-5 criteria (APA, 2013). In total, 39.3\% of participants reported symptoms meeting diagnostic criteria for at least one other psychological disorder; $16.1 \%$ of participants reported symptoms meeting diagnostic criteria for two or more psychological disorders. Among this subsample $(n=22)$, the following disorders were endorsed: $\operatorname{MDD}(n=$ 17), SAD $(n=12)$, Generalized Anxiety Disorder $(n=5)$, Obsessive-Compulsive Disorder $(n=$ 
1), and a Substance Use Disorder $(n=1)$. No participants endorsed diagnostic criteria consistent with mania or a psychotic disorder.

Frequencies of specific psychological diagnoses within each study group are displayed in Table 2. As indicated by chi-square analyses, the overall presence/absence of psychological disorders (not including BN) did not differ between groups, $\chi^{2}(1)=2.70, p=.101, \phi=.219$. In addition, chi-square analyses were not significant for the presence of Generalized Anxiety Disorder, $\chi^{2}(1)=1.98, p=.160, \phi=.188$, Obsessive Compulsive Disorder, $\chi^{2}(1)=1.02, p=$ $.313 \phi=.135$, and Substance Use Disorder, $\chi^{2}(1)=1.02, p=.313, \phi=.135$. Chi-square analyses were significant for the presence of MDD, $\chi^{2}(1)=14.28, p<.001, \phi=.505$, and SAD, $\chi^{2}(1)=$ $6.79, p=.009, \phi=.35$, such that the proportions of individuals in the $\mathrm{BN}$ group who endorsed MDD or SAD were significantly greater than the proportions of individuals in the NED group that endorsed MDD or SAD. The presence of unexpected panic attacks (not a DSM diagnosis but an important variable to consider in $\mathrm{CO}_{2}$ research; Perna, Gabriele, et al., 1995) also varied between the two groups as revealed by a significant chi-square, $\chi^{2}(1)=6.49, p=.011, \phi=.340$. This result indicated that the proportion of individuals in the $\mathrm{BN}$ group who endorsed a history of unexpected panic attacks was significantly greater than that in the NED group.

Self-report measures. Scores on all of the following continuous variables were normally distributed within groups. Table 2 displays the means and SDs of all symptom measures, separated by study group. Current stress level was assessed by a measure of stress appraisal (PSS) and a measure of recent stressful life events (HRSI). HRSI scores were similar between groups, $t(54)=1.41, p<.001, r_{\mathrm{pb}}=.19$. PSS scores were significantly greater in the BN group compared to the NED group, $t(54)=6.85, p<.001, r_{\mathrm{pb}}=.68$. Scores on the following psychological symptom measures were also significantly greater in the BN group compared to 
the NED group, as revealed by $t$-tests (which used adjusted degrees of freedom when Levene's test detected unequal variances): SPIN, $t(54)=3.59 p=.001, r_{\mathrm{pb}}=.44 ;$ DASS-21-D,$t(54)=$ $5.74, p<.001, r_{\mathrm{pb}}=.66 ;$ STICSA-State, $t(43.01)=6.49, p<.001, r_{\mathrm{pb}}=.66 ;$ STICSA-Trait version, $t(43.09)=7.55, p<.001, r_{\mathrm{pb}}=.72 ; \mathrm{ASI}-3, t(43.97)=6.31, p<.001, r_{\mathrm{pb}}=.65 ; \mathrm{BFNE}$, $t(54)=4.27, p<.001, r_{\mathrm{pb}}=.50 ;$ and SAAS, $t(38.84)=5.81, p<.001, r_{\mathrm{pb}}=.62$

Perfectionism was assessed by multiple questionnaires. The BN group scored significantly higher than the NED group on the total PAPS, $t(54)=5.88, p<.001$, and the PAPSworry about imperfection subscale, $t(54)=6.34, p<.001, r_{\mathrm{pb}}=.65$. Scores on the PAPS-hope for perfection subscale were not significantly different between groups, $t(54)=1.62, p=.110, r_{\mathrm{pb}}$ $=.22$. The BN group also scored significantly higher than the NED group on the total HMPS, $t(54)=3.83=2, p<.001, r_{\mathrm{pb}}=.46$, and each HMPS subscale: self-oriented, $t(54)=3.09, p<$ $.001, r_{\mathrm{pb}}=.39$, other-oriented, $t(54)=2.85, p=.006, r_{\mathrm{pb}}=.36$, and socially-prescribed, $t(54)=$ $3.70, p=.001, r_{\mathrm{pb}}=.45$. Moreover, the $\mathrm{BN}$ group scored significantly higher than the NED group on the total FMPS, $t(54)=3.91, p<.001, r_{\mathrm{pb}}=.47$, and four of the six FMPS subscales: concern over mistakes, $t(54)=3.88, p<.001, r_{\mathrm{pb}}=.47$, doubts about actions, $t(54)=3.49, p=$ $.000, r_{\mathrm{pb}}=.43$, personal standards $t(54)=3.10, p=.003, r_{\mathrm{pb}}=.39$, organization, $t(54)=2.02, p=$ $.048, r_{\mathrm{pb}}=.27$. Group means did not differ on the remaining two FMPS subscales: parental expectations, $t(54)=1.63, p=.108, r_{\mathrm{pb}}=.22$, parental criticism, $t(54)=1.36, p=.180, r_{\mathrm{pb}}=.18$.

A composite score was calculated from the FMPS and the HMPS for two perfectionism groups (as per factor analysis performed by Bieling et al., 2004), to differentiate between adaptive and maladaptive perfectionism. An analysis of group means demonstrated that the BN group scored higher than the NED group on both Maladaptive Evaluative Concerns, $t(54)=3.88$, $p<.001, r_{\mathrm{pb}}=.47$, and Positive Striving, $t(54)=3.76, p<.001, r_{\mathrm{pb}}=.46$. 
Table 1

Sample Demographics, Separated by Study Group

\begin{tabular}{|c|c|c|}
\hline Variable & $\mathrm{BN}(n=28)$ & $\operatorname{NED}(n=28)$ \\
\hline Age in years - $M(S D)$ & $26.11(10.38)$ & $26.25(9.35)$ \\
\hline Body Mass Index - $M(S D)$ & $23.37(5.75)$ & $22.46(4.67)$ \\
\hline \multicolumn{3}{|l|}{ Marital Status - Frequency (\%) } \\
\hline Single & $23(85.7)$ & $20(71.4)$ \\
\hline Married or Common Law & $3(10.7)$ & $6(21.4)$ \\
\hline Divorced or Separated & $1(3.6)$ & $1(3.6)$ \\
\hline Widowed & $0(0)$ & $1(3.6)$ \\
\hline Presence of Children - Frequency (\%) & $0(0)$ & $4(14.3)$ \\
\hline Postsecondary Education - Frequency (\%) & $16(57.1)$ & $18(64.3)$ \\
\hline Annual Household Income $\$-M(S D)^{\text {a }}$ & $51,833(34,778)$ & $67,500(39,921)$ \\
\hline Canadian-Born - Frequency $(\%)$ & $17(60.1)$ & $14(68.0)$ \\
\hline \multicolumn{3}{|l|}{ Ethnicity - Frequency (\%) } \\
\hline White & $11(39.3)$ & $7(25.0)$ \\
\hline Aboriginal & $0(0)$ & $1(3.6)$ \\
\hline East Indian & $2(7.1)$ & $4(14.3)$ \\
\hline Black & $2(7.1)$ & $2(7.1)$ \\
\hline South Asian & $4(14.3)$ & $1(3.6)$ \\
\hline East Asian & $5(17.8)$ & $8(28.6)$ \\
\hline Arab or West Asian & $1(3.6)$ & $1(3.6)$ \\
\hline Latin American/Hispanic & $1(3.6)$ & $2(7.1)$ \\
\hline Mixed ethnicity & $1(3.6)$ & $2(7.1)$ \\
\hline Prefer not to say & $1(3.6)$ & $0(0)$ \\
\hline
\end{tabular}

${ }^{a}$ Four participants in the HC group and 10 participants in the BN group declined to report annual household income and thus the reported $M(S D)$ is based on a smaller sample. $(n=42)$. 
Table 2

Baseline Clinical Characteristics of the Sample, Separated by Study Group

\begin{tabular}{|c|c|c|}
\hline Variable & $\mathrm{BN}(n=28)$ & $\operatorname{NED}(n=28)$ \\
\hline \multicolumn{3}{|l|}{ DSM-5 diagnoses - frequency $(\%)^{\mathrm{a}}$} \\
\hline Major Depressive Disorder *** & $15(53.6)$ & $2(7.1)$ \\
\hline Social Anxiety Disorder** & $10(35.7)$ & $2(7.1)$ \\
\hline Obsessive Compulsive Disorder & $1(3.6)$ & $0(0)$ \\
\hline Generalized Anxiety Disorder & $4(14.3)$ & $1(3.6)$ \\
\hline Substance Use Disorder & $1(3.6)$ & $0(0)$ \\
\hline Bulimia Nervosa ${ }^{* * *}$ & $28(100)$ & $0(0)$ \\
\hline \multicolumn{3}{|l|}{ Panic history - frequency (\%) } \\
\hline History of unexpected panic attacks* & $8(28.6)$ & $1(3.6)$ \\
\hline \multicolumn{3}{|l|}{ Scores on self-report measures $-M(S D)$} \\
\hline EAT-26*** & $30.42(13.32)$ & $5.18(4.10)$ \\
\hline EDI - Bulimia subscale*** & $10.68(5.91)$ & $0.18(0.61)$ \\
\hline EDI - Drive for Thinness subscale ${ }^{* * *}$ & $12.14(4.66)$ & $1.25(2.32)$ \\
\hline EDI - Body Dissatisfaction subscale $* * *$ & $16.43(6.97)$ & $4.43(4.83)$ \\
\hline PSS $* * *$ & $24.54(6.03)$ & $12.96(6.60)$ \\
\hline HRSI & $229.96(101.21)$ & $185.86(130.74)$ \\
\hline DASS-21-D*** & $17.07(11.14)$ & $2.79(3.82)$ \\
\hline STICSA-S $* * *$ & $43.79(11.86)$ & $27.04(6.80)$ \\
\hline STICSA-T*** & $44.93(10.83)$ & $26.93(6.47)$ \\
\hline ASI- $3^{b * * *}$ & $31.25(14.79)$ & $8.70(1.67)$ \\
\hline SPIN** & $21.36(11.46)$ & $10.79(10.55)$ \\
\hline $\mathrm{BFNE} * * *$ & $43.00(10.67)$ & $31.39(9.63)$ \\
\hline SAAS $* * *$ & $47.93(17.01)$ & $27.21(8.19)$ \\
\hline PAPS $* * *$ & $46.64(9.53)$ & $32.54(8.40)$ \\
\hline Worry about imperfection $* * *$ & $26.29(7.15)$ & $14.00(7.36)$ \\
\hline
\end{tabular}


Table 2 Continued

Hope for perfection

$\operatorname{HMPS} * * *$

Self oriented perfectionism***

Other oriented perfectionism***

Socially prescribed perfectionism***

FMPS $* * *$

Concern over mistakes***

Doubts about actions $* * *$

Parental expectations

Parental criticism

Personal standards***

Organization***

Perfectionism - Maladaptive concerns***

Perfectionism - Positive striving***
$20.36(4.88)$

$18.54(3.39)$

204.43 (28.76)

$167.79(41.82)$

$76.29(16.43)$

$62.43(17.15)$

$64.29(9.27)$

$55.61(13.21)$

$63.86(12.19)$

49.75 (16.08)

120.18 (18.23)

98.07 (23.77)

$32.79(6.55)$

24.82 (8.68)

13.89 (3.76)

$10.21(4.12)$

$15.86(4.83)$

$13.82(4.49)$

12.04 (4.58)

10.36 (4.67)

25.68 (5.35)

$21.14(5.60)$

$22.68(4.80)$

$20.00(5.11)$

$150.86(24.98)$

121.64 (32.00)

112.64 (13.16)

96.75 (18.11)

${ }^{\mathrm{a}}$ One participant in the HC group endorsed symptoms consistent with MDD and SAD. Seven participants in the BN group endorsed symptoms consistent with MDD and SAD, and one participant in the BN group endorsed symptoms consistent with MDD, OCD and GAD. All other participants who endorsed diagnostic criteria for a mood, anxiety or substance use disorder endorsed a single disorder. ${ }^{\mathrm{b}}$ ASI total scores were calculated for 55 participants; $50 \%$ of data on the ASI was missing for one participant in the BN group, therefore total score could not be calculated.

Note. BN = Bulimia Nervosa; NED = non-eating disorder; DSM-5 = Diagnostic and Statistical Manual of Mental Disorders $5^{\text {th }}$ Ed.; EAT-26 = Eating Attitudes Test 26-item version; EDI = Eating Disorder Inventory; PSS = Perceived Stress Scale; HRSI = Holmes-Rahe Stress Inventory; DASS-21-D = Depression Anxiety Stress Scales 21-item version, depression subscale; STICSA-S = State Trait Inventory for Cognitive and Somatic Anxiety - State version; STICSA-T = State Trait Inventory for Cognitive and Somatic Anxiety - Trait version; ASI-3 = Anxiety Sensitivity Index $-3^{\text {rd }}$ Ed.; SPIN = Social Phobia Inventory; BFNE = Brief Fear of Negative Evaluation Scale; SAAS = Social Appearance Anxiety Scale; PAPS = Physical Appearance Perfectionism Scale $;$ HMPS = Hewitt Multidimensional Perfectionism Scale $;$ FMPS $=$ Frost Multidimensional Perfectionism Scale.

$* p<.05 ; * * p<.01 ; * * * p<.001$ 


\section{Response Measurement}

Both subjective (self-report) and objective, physiological measures of response to each of the three stressors were obtained.

Subjective response. All participants rated seven items on a VAS (from $0-100$ ) immediately before and after each stressor task. All participants also rated panic symptoms on the PSL immediately before and after each stressor task. Four variables constituted measures of stress reactivity: VAS ratings of anxiety, fear, and stress, and PSL scores of panic symptoms. The three remaining variables constituted measures of eating/body image related response: hunger, desire to eat, and body dissatisfaction. Subsequent analyses considered change in each of these variables from time 1 (prestressor) to time 2 (poststressor). Table 3 displays the means and standard deviations of participants' scores on each VAS and the PSL, separated by group.

Physiological response. A subset of participants $(n=44)$ also provided physiological stress response data via measurement of cardiovascular functioning. Specifically, continuous measures of HR and BP (SYS, DIA) were recorded throughout the duration of each stress task. Data were acquired from four distinct time blocks: baseline, stressor task, postquestionnaire completion, and recovery. The baseline and recovery blocks were standardized across participants and tasks at 3 minutes and 5 minutes, respectively. The postquestionnaire completion period typically ranged between 30 and 60 seconds. The duration of the TSST was 9 minutes, and the duration of the BIS was 150 seconds. The overall duration of the $\mathrm{CO}_{2}$ task was variable across participants; timing was dependent on how well each participant understood the initial instructions and was subsequently able to inhale a full capacity breath on the first practice opportunity (or whether this practice had to be repeated). However, the $\mathrm{CO}_{2}$ breath procedure itself was standardized; all participants were asked to breathe normally while connected to the 
breathing circuit for 1 minute prior to the $\mathrm{CO}_{2}$ inhalation. Table 4 displays the means and SDs of HR, SYS-BP and DIA-BP values for each of the four time blocks, separated by group. Rather than combining data from the last two time blocks (questionnaire completion, recovery), means for each block were calculated separately and Pearson product-moment correlations were performed within groups to determine how similar HR and BP values were from these two time periods, for each stressor task. All correlations were significant at the .01 level (2-tailed); across the two groups, $r$ values ranged between $.89-.97$ for $\mathrm{CO}_{2}, .78-.96$ for the TSST, and $.61-.98$ for the BIS. Therefore, subsequent analyses considered change in HR and BP at baseline, stressor task, and recovery.

Measuring change. Residualized change in scores from time 1 to time 2 were calculated for each measure of response, in order to control for initial differences and measurement error inherent in the use of repeated measures on the same instrument (Steketee \& Chambless, 1992). These change scores were used for subsequent analyses, as required. First, they were used to examine the Pearson product-moment correlations among the seven indicators of subjective response. Table 5 displays the correlations among these seven measures of response to each stressor. Across the stressors, medium to large associations were found ( $r$ values ranging from .31 to .86$)$. Since the strength of these correlations varied across stressors, each indicator of response was retained for unique analysis.

Summary. To measure subjective response to each stressor, participants rated each variable at two time points: immediately before (time 1) and immediately after (time 2 ) the task, with post stressor rating indicating peak response. Physiological stressor response was indicated by cardiovascular measurement during two time periods: baseline (time 1) and the duration of the stressor task (time 2). Residualized change scores were used for subsequent analyses. 
Table 3

Means and Standard Deviations of Subjective Stress Response Raw Scores, Separated by Group and Stressor

\begin{tabular}{|c|c|c|c|c|}
\hline \multirow{3}{*}{ TSST } & \multirow{2}{*}{\multicolumn{2}{|c|}{$\mathrm{BN}(n=28)$}} & \multirow{2}{*}{\multicolumn{2}{|c|}{$\operatorname{NED}(n=28)$}} \\
\hline & & & & \\
\hline & Time 1 & Time 2 & Time 1 & Time 2 \\
\hline VAS-anxiety & $17.64(16.15)$ & $35.82(26.65)$ & $9.14(12.85)$ & $20.93(24.70)$ \\
\hline VAS-fear & $7.82(11.39)$ & $16.82(22.21)$ & $3.79(8.17)$ & $6.00(15.03)$ \\
\hline VAS-stress & $20.50(35.34)$ & $42.39(31.51)$ & $6.54(10.97)$ & $20.93(24.67)$ \\
\hline VAS-hunger & $29.39(26.78)$ & $29.96(29.95)$ & $25.43(27.42)$ & $17.57(21.96)$ \\
\hline VAS-desire to eat & $26.89(28.83)$ & 24.39 (31.09) & $23.32(27.15)$ & $15.75(19.70)$ \\
\hline VAS-body dissatisfaction & $41.79(35.34)$ & $34.50(36.50)$ & $8.21(15.11)$ & $4.71(9.30)$ \\
\hline PSL & $4.71(5.11)$ & $7.86(5.69)$ & $0.61(0.96)$ & $2.36(2.56)$ \\
\hline $\mathrm{CO}_{2}$ & Time 1 & Time 2 & Time 1 & Time 2 \\
\hline VAS-anxiety & $15.11(13.88)$ & $23.29(28.68)$ & $6.14(7.48)$ & $8.93(16.92)$ \\
\hline VAS-fear & $5.86(10.47)$ & $16.68(27.85)$ & $2.57(8.30)$ & $4.54(14.88)$ \\
\hline VAS-stress & $21.75(25.37)$ & $24.50(30.56)$ & $5.21(8.08)$ & $8.04(14.31)$ \\
\hline VAS-hunger & $33.29(27.45)$ & $23.18(24.00)$ & $22.96(25.13)$ & $20.64(25.89)$ \\
\hline VAS-desire to eat & $27.32(26.42)$ & $18.64(23.08)$ & $24.64(26.88)$ & $19.07(28.23)$ \\
\hline VAS-body dissatisfaction & $38.64(36.42)$ & $30.29(34.87)$ & $6.75(14.67)$ & $4.11(10.32)$ \\
\hline PSL & $3.5(3.78)$ & $7.29(6.31)$ & $0.75(1.08)$ & $3.43(3.27)$ \\
\hline BIS & Time 1 & Time 2 & Time 1 & Time 2 \\
\hline VAS-anxiety & $20.04(21.16)$ & $22.07(20.61)$ & $8.82(14.41)$ & $5.54(12.47)$ \\
\hline VAS-fear & $10.00(18.77)$ & $12.00(19.36)$ & $3.89(13.21)$ & $2.36(7.79)$ \\
\hline VAS-stress & $21.32(24.84)$ & $30.39(32.30)$ & $5.71(8.56)$ & $4.43(8.83)$ \\
\hline VAS-hunger & $32.71(28.80)$ & $26.86(29.06)$ & $25.57(29.40)$ & $22.86(27.87)$ \\
\hline VAS-desire to eat & $26.71(28.26)$ & $18.46(25.01)$ & $25.18(29.79)$ & $21.96(26.61)$ \\
\hline VAS-body dissatisfaction & $42.11(36.53)$ & $56.00(38.74)$ & $8.43(20.21)$ & $9.93(17.95)$ \\
\hline PSL & $4.18(4.51)$ & $4.96(5.24)$ & $0.57(0.92)$ & $0.50(1.04)$ \\
\hline
\end{tabular}

Note. $\mathrm{BN}=$ Bulimia Nervosa; $\mathrm{NED}=$ non-eating disorder; TSST $=$ Trier Social Stress Test $; \mathrm{CO}_{2}=$ carbon dioxide; BIS = body image stressor; VAS = visual analogue scale; PSL = Panic Symptoms List . 
Table 4

Means and Standard Deviations of Physiological (Cardiovascular) Stress Response Measures for Each Stressor

\begin{tabular}{|c|c|c|c|c|c|c|}
\hline \multirow[b]{2}{*}{ TSST } & \multicolumn{3}{|c|}{$\mathrm{BN}(n=28)$} & \multicolumn{3}{|c|}{$\operatorname{NED}(n=28)$} \\
\hline & HR & SYS-BP & DIA-BP & HR & SYS-BP & DIA-BP \\
\hline Baseline & $70.38(12.42)$ & $106.53(15.33)$ & $66.62(9.80)$ & 71.76 (8.59) & $106.21(12.82)$ & $67.48(7.60)$ \\
\hline Stressor & $76.55(12.33)$ & $104.64(16.56)$ & $69.08(12.05)$ & $78.45(10.15)$ & $107.10(17.46)$ & $70.00(8.87)$ \\
\hline Post-measures & $70.33(11.70)$ & $106.73(23.69)$ & $66.21(15.88)$ & $71.90(9.93)$ & $104.12(15.58)$ & $64.24(8.46)$ \\
\hline Recovery & $68.76(11.09)$ & 106.99 (19.79) & $68.14(14.86)$ & $71.03(9.14)$ & $102.44(15.29)$ & $63.84(8.88)$ \\
\hline $\mathrm{CO}_{2}$ & HR & SYS-BP & DIA-BP & HR & SYS-BP & DIA-BP \\
\hline Baseline & $71.08(11.54)$ & $108.80(17.67)$ & $67.20(10.83)$ & 71.49 (7.63) & $105.61(13.74)$ & $66.33(9.91)$ \\
\hline Stressor & 73.53 (9.91) & $108.86(20.24)$ & $67.71(10.46)$ & 73.37 (8.57) & $104.77(17.21)$ & $66.27(8.97)$ \\
\hline Post-measures & $69.69(11.25)$ & $105.58(22.26)$ & $63.79(11.06)$ & 71.74 (8.97) & 105.37 (16.69) & $62.80(8.05)$ \\
\hline Recovery & $71.21(12.51)$ & $104.38(16.91)$ & $64.62(10.34)$ & $70.52(8.10)$ & $102.51(17.82)$ & $62.55(8.80)$ \\
\hline BIS & HR & SYS-BP & DIA-BP & $\mathrm{HR}$ & SYS-BP & DIA-BP \\
\hline Baseline & 70.99 (10.77) & $106.33(21.05)$ & $67.75(10.32)$ & $71.73(8.10)$ & $105.69(12.84)$ & $65.77(8.60)$ \\
\hline Stressor & $69.76(10.73)$ & $104.30(21.01)$ & $65.91(9.97)$ & 70.87 (8.38) & $104.54(14.29)$ & $64.14(9.08)$ \\
\hline Post-measures & 72.87 (10.62) & $105.62(20.81)$ & $65.89(10.05)$ & $73.74(8.19)$ & $102.84(16.36)$ & $63.29(9.45)$ \\
\hline Recovery & $69.94(11.21)$ & $104.62(20.97)$ & $64.51(10.51)$ & $71.57(8.16)$ & $101.34(13.86)$ & $61.92(8.92)$ \\
\hline
\end{tabular}

Note. VAS = visual analogue scale; PSL = Panic Symptoms List BP = blood pressure; HR = heart rate; DIA = diastolic;

$\mathrm{SYS}=$ systolic $; \mathrm{TSST}=$ Trier Social Stress Test $; \mathrm{CO}_{2}=$ carbon dioxide; $\mathrm{BIS}=$ body image stressor . 
Table 5

Correlations Among Subjective Stress Response Indicators (Residualized Change Scores) for Each Stressor, Across Groups

\begin{tabular}{|c|c|c|c|c|c|c|c|}
\hline TSST & & & & & & & \\
\hline & VAS-anxiety & VAS-fear & VAS-stress & VAS-hunger & VAS-eat & VAS-body & PSL \\
\hline VAS-anxiety & -- & & & & & & \\
\hline VAS-fear & $.65^{* *}$ & -- & & & & & \\
\hline VAS-stress & $.78 * *$ & $.53 * *$ & -- & & & & \\
\hline VAS-hunger & -.02 & .21 & .15 & -- & & & \\
\hline VAS-eat & -.22 & .02 & -.09 & $.86^{* *}$ & -- & & \\
\hline VAS-body & -.22 & $.29 *$ & -.04 & -.07 & -.09 & -- & \\
\hline PSL & $.49^{* *}$ & .22 & $.58 * *$ & .23 & .07 & -.21 & -- \\
\hline \multicolumn{8}{|l|}{$\mathrm{CO}_{2}$} \\
\hline & VAS-anxiety & VAS- fear & VAS-stress & VAS-hunger & VAS-eat & VAS-body & PSL \\
\hline VAS-anxiety & -- & & & & & & \\
\hline VAS-fear & $.78 * *$ & -- & & & & & \\
\hline VAS-stress & $.67 * *$ & $.59 * *$ & -- & & & & \\
\hline VAS-hunger & -.13 & -.13 & -.10 & -- & & & \\
\hline VAS-eat & -.03 & -.08 & -.04 & $.85^{* *}$ & -- & & \\
\hline VAS-body & $-.34 *$ & $-.31 *$ & -.14 & $.26^{*}$ & .19 & -- & \\
\hline PSL & $.53 * *$ & $.39 * *$ & $.58 * *$ & -.09 & -.09 & -.17 & -- \\
\hline
\end{tabular}


Table 5 Continued

BIS

VAS-anxiety VAS-fear VAS-stress VAS-hunger VAS-eat VAS-body

\section{VAS-anxiety}

VAS-fear

$.51 * *$

VAS-stress

$.63^{* *}$

.01

$-.06$

.03

.10

VAS-eat

$.39 * *$

.14

.15

.21

VAS-body

$.31^{*}$

$.50 * *$

$.43^{* *}$

.08

$-.13$

PSL

$.34 *$

.05

.21

.26

Note. VAS = visual analogue scale; PSL = Panic Symptoms List TSST = Trier Social Stress Test $; \mathrm{CO}_{2}=$ carbon dioxide; BIS = body image stressor.

$* p<.05 ; * * p<.01$ 


\section{Manipulation Checks}

Within-subjects change in stress response indicators, across groups. Within-subject

analyses were performed across the two study groups (i.e., all participants) to ensure that each stressor task indeed provoked significant change, irrespective of the direction of change.

Subjective stress response. Paired-samples $t$-tests were performed to examine withinsubjects changes from time 1 to time 2 in each of the four subjective stress reactivity indicators:

(1) VAS ratings of anxiety, fear, and stress, (2) PSL scores of panic symptoms.

Table 6 displays means and SDs of raw scores (across all participants) for each of the aforementioned subjective stress response indicators at each measurement point, $t$ statistics, associated $p$ values and Cohen's $d$ effect size estimates (Cohen, 1988, 1992).

For the TSST, there was a main effect of time on all four subjective stress response indicators; participants reported changes in anxiety, fear, stress and panic symptoms from time 1 to time 2. For $\mathrm{CO}_{2}$, there was a main effect of time on fear and panic symptoms; participants reported significant change in fear and panic symptoms from time 1 to time 2 . For the BIS, there was no main effect of time on any of the four subjective stress response indicators.

Physiological stress response. Repeated measures analyses of variance (ANOVA) were performed to examine changes across three time blocks (baseline, stressor task, recovery) in the following physiological stress reactivity indicators: (1) HR, (2) SYS-BP, (2) DIA-BP. Values from two time blocks (baseline, stressor task) were used to calculate change scores (i.e., stressor response). Values from the recovery period were used to investigate whether participants' stress levels returned to baseline following each stressor task (i.e., during the recovery period).

Table 7 displays means and SDs of heart rate and blood pressure values (across all participants) during each of the three aforementioned time blocks, and the overall ANOVA 
results: $F$ statistics, associated $p$ values and partial eta squared association indices. A Bonferroni correction for multiple comparisons was implemented to interpret the overall ANOVA. Pairwise comparisons were subsequently evaluated, results of which are displayed in Table 8.

For the TSST, there were main effects of time on HR and DIA-BP from time 1 to time 2 and from time 2 to time 3 . For $\mathrm{CO}_{2}$, there was a main effect of time on HR from time 1 to time 2. There were also main effects of SYS-BP and DIA-BP comparing time 1 with time 3 . From time 2 to time 3, there were main effects of all three cardiovascular measurements (HR, SYS-BP, DIA-BP). For the BIS, there was a main effect of time on HR and DIA-BP from time 1 to time 2, and the effect of time on SYS-BP was marginally significant from time 1 to time 2 . There was also a main effect of DIA-BP comparing time 1 with time 3 . From time 2 to time 3 , there was a main effect of DIA-BP.

Group specific within-subjects change in response indicators. Within-subject analyses were also performed within each study group to investigate group-specific stress reactivity. To test specific study hypotheses, it was necessary to examine whether each stressor task indeed provoked significant change in response indicators (both stress reactivity and body image/eating related responses) within the specific study groups.

Psychological stress response. Table 9 displays the results of paired samples $t$-tests within each study group, including $t$ statistics, associated $p$ values and Cohen's $d$ effect size estimates (Cohen, 1988, 1992) for change in each subjective stress response indicator. See Table 3 for group means and SDs of time 1 and time 2 scores.

For the TSST, participants in the BN group reported significant increases in anxiety, fear, stress, and panic symptoms from time 1 to time 2. Participants in the NED group reported significant increases in anxiety, stress, and panic symptoms from time 1 to time 2 . For $\mathrm{CO}_{2}$, 
participants in the BN group reported significant increases in fear and panic symptoms from time 1 to time 2. Participants in the NED group reported significant increases in panic symptoms from time 1 to time 2 . For the BIS, no significant changes from time 1 to time 2 were revealed within the BN group. Participants in the NED group reported significant decreases in anxiety from time 1 to time 2 .

Physiological stress response. Table 10 displays the results of paired samples $t$-tests within each study group, including $t$ statistics, associated $p$ values and Cohen's $d$ effect size estimates (Cohen, 1988, 1992) for change in each physiological stress response indicator from time 1 (baseline) to time 2 (stressor task). See Table 4 for group means and SDs of time 1 and time 2 scores. For the TSST, in each group, significant increases in HR and DIA-BP were revealed from time 1 to time 2. For $\mathrm{CO}_{2}$, in the $\mathrm{BN}$ group, a significant increase in $\mathrm{HR}$ was revealed from time 1 to time 2. In the NED group, no significant changes in cardiovascular measures were revealed. For the BIS, in each group, significant increases in DIA-BP were revealed from time 1 to time 2.

Imaginal exposure during the BIS. Participants were asked to imagine themselves wearing the static images of clothing articles that were presented to them in a digital slideshow format during the BIS task. Following this task, as a manipulation check, participants were asked to rate the extent to which they imagined themselves wearing the clothing presented in the slideshow on a five point scale from not at all to the entire time. In the $\mathrm{BN}$ group, $71 \%$ of participants reported imagining the clothing on themselves for the entire time or most of the time, compared to $82 \%$ of participants in the NED group. The remaining participants reported imagining the clothing on themselves for half the time or a little of the time. No participant endorsed none of the time. The proportions of each response were not significantly different between groups, $\chi^{2}(4)=4.89, p=.299, \phi_{c}=.295$. 
Table 6

Paired Samples T-Tests of Subjective Stress Response Raw Scores at Time 1 and Time 2, Across Groups

\begin{tabular}{|c|c|c|c|c|c|}
\hline TSST & Time 1 & Time 2 & $t(55)$ & $p$-value & $d$ \\
\hline VAS-anxiety & $13.39(15.09)$ & $28.38(26.40)$ & 4.53 & $<.001 *$ & .656 \\
\hline VAS-fear & $5.80(10.03)$ & $11.41(19.57)$ & 2.31 & $.025^{*}$ & .343 \\
\hline VAS-stress & $13.52(16.80)$ & $31.66(30.05)$ & 4.84 & $<.001 *$ & .703 \\
\hline PSL & $2.66(4.19)$ & $5.11(5.18)$ & 4.81 & $<.001 *$ & .663 \\
\hline \multicolumn{6}{|l|}{$\mathrm{CO}_{2}$} \\
\hline VAS-anxiety & $10.63(11.93)$ & $16.11(24.43)$ & 2.00 & .051 & .315 \\
\hline VAS-fear & $4.21(9.51)$ & $10.61(22.96)$ & 2.62 & $.011 *$ & .471 \\
\hline VAS-stress & $13.48(20.43)$ & $16.27(25.06)$ & 2.46 & .230 & .168 \\
\hline PSL & $2.13(3.08)$ & $5.36(5.35)$ & 5.65 & $<.001 *$ & .857 \\
\hline \multicolumn{6}{|l|}{ BIS } \\
\hline VAS-anxiety & $14.43(18.81)$ & $13.80(18.83)$ & -0.38 & .706 & -.051 \\
\hline VAS-fear & 6.95 (16.37) & $7.18(15.41)$ & 0.12 & .909 & .015 \\
\hline VAS-stress & $13.52(20.02)$ & $17.41(26.87)$ & 1.40 & .166 & .196 \\
\hline PSL & $2.38(3.71)$ & $2.73(4.37)$ & 1.00 & .322 & .135 \\
\hline
\end{tabular}

Note. TSST = Trier Social Stress Test $; \mathrm{CO}_{2}=$ carbon dioxide; BIS = body image stressor; VAS = visual analogue scale; $\mathrm{PSL}=$ Panic Symptoms List.

Note. Values are shown as $M(S D)$.

Note. Time 1 = prestressor; Time 2 = poststressor.

* indicates a significant effect. 
Table 7

Repeated Measures Analysis of Variance of Mean HR and BP Values Measured During a Baseline Period, Stressor Task, and Recovery Period, Across Groups

\begin{tabular}{ccccccc}
\hline TSST & Time 1 & Time 2 & Time 3 & $F(2,86)$ & $p$-value & $\eta_{\mathrm{p}}{ }^{2}$ \\
\hline HR & $71.96(10.49)$ & $77.77(11.78)$ & $69.95(10.06)$ & 36.93 & $<.001^{*}$ & .462 \\
SYS-BP & $106.36(13.90)$ & $105.92(16.89)$ & $104.62(17.52)$ & 0.54 & .587 & .012 \\
DIA-BP & $67.07(8.63)$ & $69.56(10.39)$ & $65.90(12.15)$ & $5.64^{\mathrm{a}}$ & $.011^{*}$ & .116 \\
\hline $\mathrm{CO}_{2}$ & & & & & \\
\hline HR & $71.29(9.58)$ & $73.45(9.13)$ & $70.85(10.32)$ & $10.30^{\mathrm{a}}$ & $<.001^{*}$ & .196 \\
SYS-BP & $107.13(15.73)$ & $106.72(18.61)$ & $103.40(17.21)$ & 8.09 & $.001^{*}$ & .158 \\
DIA-BP & $66.74(8.90)$ & $66.96(9.62)$ & $63.54(9.51)$ & 18.01 & $<.001^{*}$ & .295 \\
\hline BIS & & & & & \\
\hline HR & $71.38(9.36)$ & $70.34(9.48)$ & $70.80(9.65)$ & 3.05 & .052 & .066 \\
SYS-BP & $105.99(17.05)$ & $104.42(17.60)$ & $102.91(19.48)$ & $2.71^{\mathrm{a}}$ & .093 & .059 \\
DIA-BP & $66.72(9.40)$ & $64.98(9.44)$ & $63.16(9.69)$ & 20.13 & $<.001^{*}$ & .319
\end{tabular}

\footnotetext{
${ }^{a}$ Mauchley's Test of Sphericity was significant, therefore $d f$ was adjusted using a Greenhouse-Geisser adjustment as follows: $F(1.48,63.69)=5.64 ; F(1.58,68.04)=10.30 ; F(1.38,59.44)=2.71$

Note. TSST = Trier Social Stress Test $\mathrm{CO}_{2}=$ carbon dioxide; $\mathrm{BIS}=$ body image stressor; VAS = visual analogue scale; PSL = Panic Symptoms List; HR = heart rate; BP = blood pressure; SYS = systolic; DIA $=$ diastolic.

Note. $N=44$; values are shown as $M(S D)$.

Note. Time 1 = baseline; Time 2 = stressor task; Time 3 = recovery.

$*$ indicates a significant effect.
} 
Table 8

P-Values and Cohen's d Effect Sizes for Pairwise Comparisons of Mean HR and BP Values, Across Groups

\begin{tabular}{llll}
\hline TSST & BL, Task & BL, Recovery & Task, Recovery \\
\hline HR & $<.001^{*}, d=1.046$ & $.176, d=-.208$ & $<.001^{*}, d=-1.091$ \\
SYS-BP & $.798, d=-.040$ & $.311, d=-.162$ & $.483, d=-.106$ \\
DIA-BP & $.001^{*}, d=.558$ & $.377, d=-.145$ & $.004^{*}, d=-.463$ \\
\hline $\mathrm{CO}_{2}$ & & & \\
\hline HR & $.004^{*}, d=.458$ & $.312, d=-.158$ & $<.001^{*}, d=-.641$ \\
SYS-BP & $.683, d=-.069$ & $.002^{*}, d=-.513$ & $.001^{*}, d=-.564$ \\
DIA-BP & $.741, d=.053$ & $<.001^{*}, d=-.687$ & $<.001^{*}, d=-.917$ \\
\hline BIS & & $.210, d=.196$ & $.212, d=-.193$ \\
\hline HR & $.023^{*}, d=-.360$ & $.347, d=-.143$ & $.050, d=-.318$ \\
SYS-BP & $.050, d=-.305$ & $<.001^{*}, d=-.584$ & $<.001^{*}, d=-.874$ \\
DIA-BP & $.005^{*}, d=-.448$ & &
\end{tabular}

Note. TSST = Trier Social Stress Test $\mathrm{CO}_{2}=$ carbon dioxide; $\mathrm{BIS}=$ body image stressor; $\mathrm{BL}=$ baseline; $\mathrm{HR}=$ heart rate; $\mathrm{BP}=$ blood pressure; $\mathrm{SYS}=$ systolic; $\mathrm{DIA}=$ diastolic .

Note. $N=44$; values are shown as $p$, Cohen's $d$.

* indicates a significant effect. 
Table 9

Paired Samples T-Tests of Subjective Stress Response Raw Scores at Time 1 and Time 2, Separated by Group

\begin{tabular}{|c|c|c|c|c|c|c|}
\hline \multirow{3}{*}{ TSST } & \multirow{2}{*}{\multicolumn{3}{|c|}{ BN Group $(n=56)$}} & & & \\
\hline & & & & \multicolumn{3}{|c|}{ NED Group $(n=56)$} \\
\hline & $t(27)$ & $p$-value & $d$ & $t(27)$ & $p$-value & $d$ \\
\hline VAS-anxiety & 3.64 & $.001^{*}$ & .718 & 2.72 & $.011 *$ & .525 \\
\hline VAS-fear & 2.10 & $.045^{*}$ & .428 & 1.01 & .324 & .224 \\
\hline VAS-stress & 3.56 & $.001^{*}$ & .532 & 3.35 & $.002 *$ & .733 \\
\hline PSL & 3.43 & $.002^{*}$ & .654 & 4.14 & $<.001 *$ & .993 \\
\hline VAS-body dissatisfaction & -1.77 & .089 & -.335 & -2.47 & $.020 *$ & -.717 \\
\hline VAS-hunger & 0.14 & .892 & .026 & -2.21 & $.035^{*}$ & -.435 \\
\hline VAS-desire to eat & -0.65 & .521 & -.123 & 1.63 & .114 & .319 \\
\hline \multicolumn{7}{|l|}{$\mathrm{CO}_{2}$} \\
\hline VAS-anxiety & 1.65 & .110 & .355 & 1.16 & .256 & .303 \\
\hline VAS-fear & 2.42 & $.023 *$ & .598 & 1.19 & .244 & .328 \\
\hline VAS-stress & 0.64 & .530 & .123 & 1.70 & .101 & .439 \\
\hline PSL & 3.97 & $<.001 *$ & .841 & 4.22 & $<.001 *$ & .910 \\
\hline VAS-body dissatisfaction & -1.87 & .072 & -.355 & -2.16 & $.040 *$ & -.542 \\
\hline VAS-hunger & -2.76 & $.010^{*}$ & -.529 & -0.68 & .500 & -.129 \\
\hline VAS-desire to eat & -3.07 & $.005^{*}$ & -.595 & -2.11 & $.044 *$ & -.400 \\
\hline \multicolumn{7}{|l|}{ BIS } \\
\hline VAS-anxiety & 0.73 & .475 & .137 & -2.00 & $.055^{*}$ & -.387 \\
\hline VAS-fear & 0.52 & .609 & .098 & -1.29 & .207 & -.461 \\
\hline VAS-stress & 1.72 & .097 & .334 & -1.03 & .312 & -.194 \\
\hline PSL & 1.13 & .267 & .217 & -0.44 & .663 & -.082 \\
\hline VAS-body dissatisfaction & 2.60 & $.015^{*}$ & .492 & 0.84 & .408 & .163 \\
\hline VAS-hunger & -2.62 & $.014^{*}$ & -.493 & -1.56 & .131 & -.299 \\
\hline VAS-desire to eat & -1.77 & .088 & -.337 & -1.99 & .056 & -.404 \\
\hline
\end{tabular}

Note. $\mathrm{BN}=$ Bulimia Nervosa; NED = non-eating disorder; TSST = Trier Social Stress Test $\mathrm{CO}_{2}=$ carbon dioxide; BIS = body image stressor; VAS = visual analogue scale; PSL = Panic Symptoms List .

* indicates a significant effect. 
Table 10

P-values and Cohen's d Effect Sizes for Pairwise Comparisons of Mean HR and BP Values from Baseline to Stress Task, Separated by Group

\begin{tabular}{|c|c|c|}
\hline & BN Group $(n=21)$ & NED Group $(n=23)$ \\
\hline TSST & BL to Task & BL to Task \\
\hline HR & $<.001^{*}, d=1.224$ & $.001^{*}, d=.985$ \\
\hline SYS-BP & $.534, d=-.138$ & $.630, d=.119$ \\
\hline DIA-BP & $.031^{*}, d=.570$ & $.020^{*}, d=.541$ \\
\hline \multicolumn{3}{|l|}{$\mathrm{CO}_{2}$} \\
\hline HR & $.035^{*}, d=.521$ & $.063, d=.416$ \\
\hline SYS-BP & $.971, d=.009$ & $.518, d=-.164$ \\
\hline DIA-BP & $.586, d=.123$ & $.944, d=-.016$ \\
\hline \multicolumn{3}{|l|}{ BIS } \\
\hline HR & $.128, d=.348$ & $.077, d=-.394$ \\
\hline SYS-BP & $.143, d=-.333$ & $.208, d=-.286$ \\
\hline DIA-BP & $.048^{*}, d=-.462$ & $.054^{*}, d=-.428$ \\
\hline \multicolumn{3}{|c|}{$\begin{array}{l}\text { Note. } \mathrm{BN}=\text { Bulimia Nervosa; NED = non-eating disorder; TSST = Trier Social } \\
\text { Stress Test } ; \mathrm{CO}_{2}=\text { carbon dioxide; } \mathrm{BIS}=\text { body image stressor; } \mathrm{HR}=\text { heart rate; } \\
\mathrm{BP}=\text { blood pressure; } \mathrm{SYS}=\text { systolic; } \mathrm{DIA}=\text { diastolic; } \mathrm{BL}=\text { baseline. } \\
\text { Note. } N=44 ; \text { values are shown as } p \text {, Cohen's } d . \\
* \text { indicates a significant effect. }\end{array}$} \\
\hline
\end{tabular}




\section{Hypothesis 1: Comparison of Response to Stressors in Participants With and Without BN}

\subsection{Group comparisons of subjective response to stressors. Independent samples $t$ -}

tests were performed to examine group differences in subjective response to each stressor task. Refer to Table 3 for means and SDs of raw scores for each response indicator, measured at time 1 and time 2 (pre and poststressor) and separated by group, from which residualized change scores were calculated. Table 11 displays the results of each $t$-test, including degrees of freedom, $t$ statistics, associated $p$ values and Cohen's $d$ effect size estimates (Cohen, 1988, 1992).

For the TSST, findings revealed significant between group differences in panic symptoms, rated on the PSL, from pre to poststressor. Specifically, participants in the BN group had a significantly greater increase in panic symptoms from pre to poststressor, compared to the NED group. No other significant between group differences were found. For $\mathrm{CO}_{2}$, findings revealed no significant between group differences in any indicators of subjective response to this stressor. For the BIS, findings revealed significant between group differences in VAS anxiety ratings, stress ratings, and body dissatisfaction ratings from pre to poststressor. Specifically, participants in the BN group had significantly greater increases in VAS ratings of anxiety, stress, and body dissatisfaction from pre to poststressor, compared to the NED group. No other significant between group differences were found.

\subsection{Magnitude of group differences in subjective response to stressors. Effect size}

estimates of between-group effects were examined in order to compare the magnitude of group differences in subjective response to each stressor (see Table 11). Overall, the BIS provoked the strongest between-group differences in stress reactivity (i.e., anxiety, fear, stress, and panic symptoms) and body dissatisfaction, with effect sizes in the medium to large range. The TSST 
provoked the strongest between-group differences in hunger and desire to eat, with effect sizes in the small to medium range.

1.3. Group comparisons of physiological response to stressors. Independent samples $t$ tests were performed to examine group differences in physiological (cardiovascular) response to each stressor task. Refer to Table 4 for means and SDs of mean HP and BP values, measured during time 1 and time 2 (baseline and stressor task) and separated by group, from which residualized change scores were calculated. Table 12 displays the results of each $t$-test, including degrees of freedom, $t$ statistics, associated $p$ values and Cohen's $d$ effect size estimates (Cohen, 1988, 1992). Findings revealed no significant between group differences on any cardiovascular measure of stress response.

\subsection{Magnitude of group differences in physiological response to stressors. Effect size} estimates of between-group effects, as indicated in Table 12, were examined in order to compare the magnitude of group differences in physiological response to each stressor. All effect size estimates were trivial (below $d=.2$ ) except for SYS-BP response to the TSST (a small effect, $d$ $=.245)$, and therefore it was not logical to examine whether the difference in magnitude of HR and $\mathrm{BP}$ responses were most pronounced with the BIS.

\subsection{Influence of the following variables on stress response: state anxiety, trait anxiety,} depression, anxiety sensitivity, stressful life events, and perceived stress. Multiple linear regressions were performed to examine whether other psychological factors could explain the significant between-group differences in stress reactivity (i.e., results of hypotheses 1.2 and 1.4). The following factors were considered and entered as predictor variables into each regression, along with group: DASS-21-D score, STICSA-State total score, STICSA-Trait total score, ASI-3 total score, HRSI total score, and PSS total score. Findings of these regression analyses, reported 
below, determined whether the group variable continued to exert an effect on stress response when the above-mentioned variables were considered. Tables 13 and 14 display the unstandardized beta coefficients and associated standard errors, as well as the standardized beta coefficients for each of the four regression analyses (i.e., one regression analysis for each significant between-group effect).

TSST - PSL scores of panic symptoms. The overall regression model to predict change in panic symptom scores from pre to post TSST accounted for $28.0 \%$ of the variance. Group was no longer a predictor of change in panic symptom scores. DASS-21-D scores and PSS scores significantly predicted change in panic symptom scores. Specifically, DASS-21-D scores predicted lower panic symptom scores, and PSS scores predicted higher panic symptom scores poststressor.

BIS - VAS-anxiety ratings. The overall regression model to predict change in anxiety ratings from pre to post BIS accounted for $21.4 \%$ of the variance. Group continued to be a significant predictor of change in anxiety ratings, with individuals in the BN group predicting greater change compared to individuals in the NED group. ASI-3 scores also significantly predicted greater change in anxiety ratings.

$B I S-V A S$-stress ratings. The overall regression model to predict change in stress ratings from pre to post-BIS accounted for $30.5 \%$ of the variance. Group was no longer a significant predictor of change in stress ratings. ASI-3 scores significantly predicted change in stress ratings.

BIS - VAS-body dissatisfaction ratings. The overall regression model to predict change in body dissatisfaction ratings from pre to post BIS accounted for $21.8 \%$ of the variance. Group was no longer a predictor of change in body dissatisfaction ratings, and none of the additional variables in the model were significant predictors. 
Table 11

Comparison of Subjective Responses to Each Stressor Between the BN Group and the NED Group

\begin{tabular}{|c|c|c|c|c|}
\hline TSST & $d f$ & $t$ & $p$-value & $d$ \\
\hline VAS-anxiety & 54 & -1.41 & .165 & .377 \\
\hline VAS-fear & 41.85 & -1.63 & .110 & .455 \\
\hline VAS-stress & 54 & -1.60 & .116 & .432 \\
\hline PSL & 39.18 & -2.05 & $.047^{*}$ & .581 \\
\hline VAS-hunger & 48.82 & -1.94 & .059 & -.577 \\
\hline VAS-desire to eat & 54 & -1.38 & .174 & -.373 \\
\hline VAS-body dissatisfaction & 30.44 & -0.51 & .614 & .158 \\
\hline \multicolumn{5}{|l|}{$\mathrm{CO}_{2}$} \\
\hline VAS-anxiety & 38.44 & -0.80 & .429 & .227 \\
\hline VAS-fear & 32.48 & -1.52 & .139 & .456 \\
\hline VAS-stress & 35.72 & -0.35 & .730 & .101 \\
\hline PSL & 54 & -0.87 & .390 & .236 \\
\hline VAS-hunger & 54 & 0.99 & .325 & -.284 \\
\hline VAS-desire to eat & 54 & 0.72 & .476 & -.192 \\
\hline VAS-body dissatisfaction & 29.04 & -0.49 & .628 & -.158 \\
\hline \multicolumn{5}{|l|}{ BIS } \\
\hline VAS-anxiety & 42.03 & -1.41 & $.013^{*}$ & .724 \\
\hline VAS-fear & 28.08 & -1.93 & .064 & .656 \\
\hline VAS-stress & 29.88 & -2.36 & $.025^{*}$ & .745 \\
\hline PSL & 29.90 & -1.56 & .130 & .490 \\
\hline VAS-hunger & 54 & 0.90 & .371 & -.243 \\
\hline VAS-desire to eat & 54 & 1.06 & .295 & -.305 \\
\hline VAS-body dissatisfaction & 32.46 & -2.76 & $.009 *$ & .831 \\
\hline
\end{tabular}

Note. TSST $=$ Trier Social Stress Test $\mathrm{CO}_{2}=$ carbon dioxide; $\mathrm{BIS}=$ body image stressor; VAS = visual analogue scale; PSL = Panic Symptoms List .

Note. NED group is coded as $0 ; \mathrm{BN}$ group is coded as 1.

* indicates a significant difference between groups. 
Table 12

Comparison of Physiological Responses to Each Stressor Between the BN Group and the NED Group

\begin{tabular}{|c|c|c|c|}
\hline TSST & $t(42)$ & $p$-value & $d$ \\
\hline HR & 0.53 & .600 & -.160 \\
\hline SYS-BP & 0.81 & .424 & -.245 \\
\hline DIA-BP & -0.001 & .999 & .000 \\
\hline \multicolumn{4}{|l|}{$\mathrm{CO}_{2}$} \\
\hline HR & -0.37 & .712 & .112 \\
\hline SYS-BP & -0.27 & .788 & .082 \\
\hline DIA-BP & -0.47 & .642 & .142 \\
\hline \multicolumn{4}{|l|}{ BIS } \\
\hline HR & 0.46 & .650 & -.139 \\
\hline SYS-BP & 0.55 & .584 & -.166 \\
\hline DIA-BP & 0.04 & .970 & -.012 \\
\hline \multicolumn{4}{|c|}{$\begin{array}{l}\text { Note. } \text { TSST = Trier Social Stress Test } ; \mathrm{CO}_{2}=\text { carbon dioxi } \\
\text { BIS = body image stressor; VAS = visual analogue scale; } \\
\text { PSL = Panic Symptoms List } ; \mathrm{HR}=\text { heart rate; } \mathrm{SYS}-\mathrm{BP}= \\
\text { systolic blood pressure; DIA = diastolic blood pressure. } \\
\text { Note. NED group is coded as } 0 ; \mathrm{BN} \text { group is coded as } 1 .\end{array}$} \\
\hline
\end{tabular}


Table 13

Regression Analysis Predicting PSL Scores in Response to the TSST by Group, Depressive Symptoms, State Anxiety, Trait Anxiety, Anxiety Sensitivity, Perceived Stress, and Stressful Life Events

\begin{tabular}{lcccc} 
& \multicolumn{4}{c}{ Predicting PSL scores } \\
\cline { 2 - 5 } & $B$ & SE $B$ & $\beta$ & $P$ \\
\hline Group & 0.45 & 0.28 & .31 & .121 \\
ASI-3 & -0.00 & 0.01 & -.01 & .969 \\
DASS-21-D & -0.05 & 0.02 & -.72 & $.003^{*}$ \\
STICSA-T & -0.02 & 0.02 & -.35 & .236 \\
STICSA-S & 0.02 & 0.02 & .43 & .158 \\
HRSI & 0.00 & 0.00 & .15 & .250 \\
PSS & 0.05 & 0.02 & .55 & $.025^{*}$ \\
& & .280 & & \\
\hline
\end{tabular}

Note. TSST = Trier Social Stress Test PSL = Panic Symptoms List; ASI-3 = Anxiety Sensitivity Index - $3^{\text {rd }}$ ed.; DASS-21-D = Depression Anxiety Stress Scales, 21-item version, depression subscale; STICSA-T = State Trait Inventory for Cognitive and Somatic Anxiety-Trait version; STICSA-S = State Trait Inventory for Cognitive and Somatic Anxiety-State version; HRSI = Holmes Rahe Stress Inventory; PSS = Perceived Stress Scale.

Note. NED group is coded as $0 ; \mathrm{BN}$ group is coded as 1.

Note. $N=56$, except for ASI-3 scores where $N=55$ due to missing data.

* indicates a significant predictor variable. 
Table 14

Regression Analyses Predicting Anxiety, Stress and Body Dissatisfaction Ratings in Response to the BIS by Group, Depressive Symptoms, State Anxiety, Trait Anxiety, Anxiety Sensitivity, Perceived Stress, and Stressful Life Events

\begin{tabular}{|c|c|c|c|c|c|c|c|c|c|c|c|c|}
\hline \multicolumn{5}{|c|}{ Predicting VAS-Anxiety } & \multicolumn{4}{|c|}{ Predicting VAS-Stress } & \multicolumn{4}{|c|}{ Predicting VAS-Body Dissatisfaction } \\
\hline Predictors & $B$ & $S E B$ & $\beta$ & $P$ & $B$ & $S E B$ & $\beta$ & $P$ & $B$ & $S E B$ & $\beta$ & $P$ \\
\hline Group & .57 & .24 & .47 & $.025^{*}$ & .04 & .29 & .03 & .885 & .19 & .23 & .17 & .421 \\
\hline ASI-3 & .02 & .01 & .39 & .066 & .03 & .01 & .57 & $.005^{*}$ & .01 & .01 & .13 & .540 \\
\hline DASS-21-D & -.002 & .01 & -.03 & .904 & -.02 & .02 & -.26 & .250 & -.004 & .01 & -.07 & .770 \\
\hline STICSA-T & .00 & .02 & .004 & .991 & .03 & .02 & .43 & .146 & -.02 & .01 & -.44 & .159 \\
\hline STICSA-S & -.02 & .02 & -.42 & .177 & -.03 & .02 & -.49 & .097 & .02 & .01 & .41 & .188 \\
\hline HRSI & .00 & .001 & -.05 & .698 & .00 & .001 & .05 & .709 & .00 & .001 & .01 & .919 \\
\hline PSS & -.01 & .02 & -.14 & .590 & .01 & .02 & .15 & .541 & .02 & .02 & .27 & .294 \\
\hline$R^{2}$ & & .214 & & & & .305 & & & & .218 & & \\
\hline
\end{tabular}

Note. BIS = body image stressor; VAS = visual analogue scale; ASI-3 = Anxiety Sensitivity Index - $3^{\text {rd }}$ ed.; DASS-21-D = Depression Anxiety Stress Scales, 21 item version, depression subscale; STICSA-T = State Trait Inventory for Cognitive and Somatic Anxiety-Trait version; STICSA-S = State Trait Inventory for

Cognitive and Somatic Anxiety-State version; HRSI = Holmes Rahe Stress Inventory; PSS = Perceived Stress Scale.

Note. $N=56$, except for ASI-3 scores where $\mathrm{N}=55$ due to missing data.

Note. NED group is coded as $0 ; \mathrm{BN}$ group is coded as 1 .

* indicates a significant predictor variable. 


\section{Hypothesis 2: Social Anxiety Symptoms and Social Anxiety-Related Factors as Predictors of Stress Response in Individuals With and Without BN.}

Multiple linear regressions were performed to predict stress response based on social anxiety symptoms and social anxiety related factors. For each statistically significant withinsubjects change from time 1 to time 2 (indicated in Tables 9 and 10), a regression was performed for group (BN or NED) and the associated response indicator. The stepwise method was used. In step 1, SPIN scores were entered. In step 2, scores on measures of social anxiety related factors were entered: BFNE, SAAS, MEC, SOP, WAI. Tables 15 to 21 display the unstandardized beta coefficients and associated standard errors, as well as the standardized beta coefficients.

TSST. In the NED group, SPIN scores significantly predicted change in stress ratings and panic symptom scores. When other predictor variables were entered into the regression, SPIN scores were no longer unique predictors of change in stress ratings and panic symptom scores. SPIN scores were marginally significant in predicting change in anxiety ratings. Also in the NED group, lower SPIN scores predicted greater DIA-BP change scores, and SPIN scores remained a significant predictor when other predictor variables were entered into the regression.

CO2. In the BN group, SPIN scores significantly predicted fear ratings; when other predictor variables were entered into the regression, SPIN scores were no longer unique predictors of change in fear ratings. Additionally, in the BN group, lower SPIN scores significantly predicted greater PSL change scores (i.e., less of a change in panic symptoms); when other predictor variables were entered into the regression, SPIN scores remained a unique predictor, and SAAS scores also contributed unique variance in predicting change in panic symptoms. No significant predictors were revealed for physiological stress reactivity. 
Table 15

Regression Analyses Predicting Anxiety, Fear, Stress, and Panic Symptoms in Response to the TSST by Social Anxiety Symptoms and Social Anxiety-Related Variables, in the BN Group

\begin{tabular}{|c|c|c|c|c|c|c|c|c|c|c|c|c|c|c|c|c|}
\hline & & \multicolumn{3}{|c|}{ Predicting VAS-Anxiety } & \multicolumn{4}{|c|}{ Predicting VAS-Fear } & \multicolumn{4}{|c|}{ Predicting VAS-Stress } & \multicolumn{4}{|c|}{ Predicting PSL scores } \\
\hline & $B$ & $S E B$ & $\beta$ & $P$ & $B$ & $S E B$ & $\beta$ & $P$ & $B$ & $S E B$ & $\beta$ & $P$ & $B$ & $S E B$ & $\beta$ & $P$ \\
\hline $\begin{array}{l}\text { BN } \\
\text { Group }\end{array}$ & & & & & & & & & & & & & & & & \\
\hline Step 1 & & & & & & & & & & & & & & & & \\
\hline SPIN & 0.00 & 0.02 & .05 & .786 & 0.03 & 0.02 & .32 & .100 & 0.01 & 0.02 & .08 & .704 & -0.02 & 0.02 & -.22 & .257 \\
\hline$R^{2}$ & & .054 & & & & .101 & & & & .006 & & & & .049 & & \\
\hline Step 2 & & & & & & & & & & & & & & & & \\
\hline SPIN & -0.04 & 0.03 & -.48 & .215 & 0.01 & 0.03 & .05 & .887 & -0.01 & 0.04 & -.09 & .835 & -0.03 & 0.03 & -.41 & .277 \\
\hline BFNE & 0.01 & 0.03 & .11 & .727 & 0.01 & 0.03 & .05 & .864 & -0.00 & 0.03 & -.01 & .976 & -0.02 & 0.03 & -.22 & .496 \\
\hline SAAS & 0.02 & 0.02 & .35 & .343 & 0.03 & 0.02 & .37 & .268 & -0.00 & 0.02 & -.06 & .877 & 0.01 & 0.02 & .17 & .641 \\
\hline MEC & 0.03 & 0.02 & .75 & .103 & 0.02 & 0.02 & .34 & .412 & 0.01 & 0.02 & .32 & .507 & 0.00 & 0.02 & .12 & .785 \\
\hline SOP & -0.03 & 0.03 & -.55 & .210 & -0.05 & 0.03 & -.69 & .094 & -0.01 & 0.03 & -.15 & .745 & 0.02 & 0.02 & .31 & .465 \\
\hline WAI & 0.01 & 0.04 & .08 & .779 & 0.02 & 0.04 & .10 & .695 & 0.04 & 0.04 & .27 & .393 & 0.01 & 0.04 & .09 & .745 \\
\hline$R^{2}$ & & .206 & & & & .337 & & & & .080 & & & & .230 & & \\
\hline
\end{tabular}

Note. TSST = Trier Social Stress Test $; \mathrm{BN}=$ Bulimia Nervosa; VAS = visual analogue scale; PSL = Panic Symptoms List ; SPIN = Social Phobia Inventory; BFNE = Brief Fear of Negative Evaluation Scale; SAAS = Social Appearance Anxiety Scale $;$ MEC = Maladaptive evaluative concerns; SOP = Self-oriented perfectionism; WAI = Worry about imperfection.

Note. NED group is coded as $0 ; \mathrm{BN}$ group is coded as 1 .

Note. $\mathrm{N}=56$. 
Table 16

Regression Analyses Predicting Anxiety, Stress, and Panic Symptoms in Response to the TSST by Social Anxiety Symptoms and Social Anxiety-Related Variables, in the NED Group

\begin{tabular}{|c|c|c|c|c|c|c|c|c|c|c|c|c|}
\hline & \multicolumn{4}{|c|}{ Predicting VAS-Anxiety } & \multicolumn{4}{|c|}{ Predicting VAS-Stress } & \multicolumn{4}{|c|}{ Predicting PSL scores } \\
\hline & $B$ & $S E B$ & $\beta$ & $P$ & $B$ & $S E B$ & $\beta$ & $P$ & & $B$ & $S E B$ & $\beta P$ \\
\hline \multicolumn{13}{|c|}{ NED Group } \\
\hline \multicolumn{13}{|l|}{ Step 1} \\
\hline SPIN & 0.03 & 0.02 & .37 & .056 & 0.03 & 0.01 & .42 & $.028^{*}$ & 0.02 & 0.01 & .40 & $.035 *$ \\
\hline$R^{2}$ & & .134 & & & & .172 & & & & .160 & & \\
\hline \multicolumn{13}{|l|}{ Step 2} \\
\hline SPIN & 0.01 & 0.02 & .18 & .497 & 0.02 & 0.02 & .26 & .329 & 0.01 & 0.01 & 1.14 & .266 \\
\hline BFNE & 0.01 & 0.03 & .10 & .723 & 0.02 & 0.02 & .56 & .396 & 0.00 & 0.01 & .29 & .775 \\
\hline SAAS & 0.07 & 0.04 & .62 & .096 & 0.02 & 0.03 & .24 & .517 & 0.01 & 0.02 & .55 & .587 \\
\hline MEC & -0.00 & 0.01 & -.14 & .733 & 0.00 & 0.01 & .05 & .901 & -0.00 & 0.01 & -.13 & .897 \\
\hline SOP & 0.01 & 0.02 & .20 & .585 & -0.01 & 0.02 & -.12 & .756 & 0.01 & 0.01 & .78 & .442 \\
\hline WAI & -0.06 & 0.04 & -.50 & .131 & -0.03 & 0.03 & -.27 & .420 & -0.02 & 0.02 & -.89 & .383 \\
\hline$R^{2}$ & & .319 & & & & .272 & & & & .270 & & \\
\hline
\end{tabular}

Note. TSST $=$ Trier Social Stress Test $;$ NED = non-eating disorder; VAS = visual analogue scale; PSL = Panic Symptoms List $;$ SPIN = Social Phobia Inventory; $\mathrm{BFNE}=$ Brief Fear of Negative Evaluation Scale; SAAS = Social Appearance Anxiety Scale; MEC = Maladaptive evaluative concerns; $\mathrm{SOP}=$ Self-oriented perfectionism; WAI $=$ Worry about imperfection .

Note. NED group is coded as $0 ; \mathrm{BN}$ group is coded as 1 .

Note. $N=56$.

* indicates a significant predictor variable. 
Table 17

Regression Analyses Predicting Heart Rate and Diastolic Blood Pressure in Response to the TSST by Social Anxiety Symptoms and Social Anxiety-Related Variables, Separated by Group

\begin{tabular}{lcccccccc}
\hline \multicolumn{3}{c}{ Predicting HR } & \multicolumn{5}{c}{ Predicting DIA-BP } \\
\hline BN Group & $B$ & $S E B$ & $\beta$ & $P$ & $B$ & $S E B$ & $\beta$ & $P$ \\
\hline Step 1 & & & & & & & & \\
SPIN & 0.01 & 0.01 & .15 & .525 & 0.02 & 0.01 & .39 & .080 \\
$R^{2}$ & & .022 & & & & .153 & & \\
Step 2 & & & & & & & & \\
SPIN & -0.00 & 0.02 & -.12 & .831 & -0.02 & 0.02 & -.63 & .174 \\
BFNE & 0.00 & 0.02 & .07 & .854 & -0.01 & 0.01 & -.03 & .921 \\
SAAS & 0.00 & 0.02 & .09 & .897 & 0.03 & 0.01 & .94 & .082 \\
MEC & 0.01 & 0.01 & .79 & .224 & 0.01 & 0.01 & .51 & .311 \\
SOP & -0.01 & 0.01 & -.47 & .448 & -0.01 & 0.01 & -.38 & .426 \\
WAI & -0.01 & 0.02 & -.13 & .733 & 0.02 & 0.02 & .25 & .399 \\
$R^{2}$ & & .172 & & & & .502 & & \\
\hline
\end{tabular}


Table 17 Continued

\begin{tabular}{|c|c|c|c|c|c|c|c|c|}
\hline \multicolumn{5}{|c|}{ Predicting HR } & \multicolumn{4}{|c|}{ Predicting DIA-BP } \\
\hline NED Group & $B$ & $S E B$ & $\beta$ & $P$ & $B$ & $S E B$ & $\beta$ & $P$ \\
\hline \multicolumn{9}{|l|}{ Step 1} \\
\hline SPIN & 0.02 & 0.01 & .39 & .080 & -0.02 & 0.01 & -.45 & $.033^{*}$ \\
\hline$R^{2}$ & & .153 & & & & .199 & & \\
\hline \multicolumn{9}{|l|}{ Step 2} \\
\hline SPIN & -0.02 & 0.02 & -.63 & .174 & -0.03 & 0.01 & -.76 & $.009 *$ \\
\hline BFNE & -0.01 & 0.01 & -.03 & .921 & -0.00 & 0.01 & -.07 & .822 \\
\hline SAAS & 0.03 & 0.01 & .94 & .082 & 0.01 & 0.02 & .18 & .625 \\
\hline MEC & 0.01 & 0.01 & .51 & .311 & 0.00 & 0.01 & .19 & .666 \\
\hline SOP & -0.01 & 0.01 & -.38 & .426 & 0.01 & 0.01 & .27 & .493 \\
\hline WAI & 0.02 & 0.02 & .25 & .399 & 0.01 & 0.02 & .19 & .567 \\
\hline$R^{2}$ & & .502 & & & & .468 & & \\
\hline
\end{tabular}

Note. TSST $=$ Trier Social Stress Test $; \mathrm{BN}=$ Bulimia Nervosa $;$ NED = non-eating disorder; $\mathrm{HR}=$ heart rate;

SYS-BP = systolic blood pressure; DIA=BP = diastolic blood pressure; $\mathrm{SPIN}=$ Social Phobia Inventory; $\mathrm{BFNE}=$

Brief Fear of Negative Evaluation Scale; SAAS = Social Appearance Anxiety Scale; MEC = Maladaptive evaluative concerns; $\mathrm{SOP}=$ Self-oriented perfectionism; WAI $=$ Worry about imperfection.

Note. NED group is coded as $0 ; \mathrm{BN}$ group is coded as 1 .

Note. $N=21$ in the BN group; $N=23$ in the NED group.

* indicates a significant predictor variable. 
Table 18

Regression Analyses Predicting Fear, Panic Symptoms and Heart Rate in Response to $\mathrm{CO}_{2}$ by Social Anxiety Symptoms and Social Anxiety-Related Variables, in the BN Group

\begin{tabular}{|c|c|c|c|c|c|c|c|c|c|c|c|c|}
\hline & \multicolumn{4}{|c|}{ Predicting VAS-Fear } & \multicolumn{4}{|c|}{ Predicting PSL scores } & \multicolumn{4}{|c|}{ Predicting HR } \\
\hline & $B$ & $S E B$ & $\beta$ & $P$ & $B$ & $S E B$ & $\beta$ & $P$ & $B$ & $S E B$ & $\beta$ & $P$ \\
\hline \multicolumn{13}{|l|}{ BN Group } \\
\hline \multicolumn{13}{|l|}{ Step 1} \\
\hline SPIN & 0.02 & 0.02 & .17 & .377 & 0.00 & 0.02 & -.00 & .983 & 0.01 & 0.01 & .29 & .196 \\
\hline$R^{2}$ & & .030 & & & & .000 & & & & .086 & & \\
\hline \multicolumn{13}{|l|}{ Step 2} \\
\hline SPIN & -0.03 & 0.03 & -.90 & .378 & -0.06 & 0.03 & -.77 & $.027 *$ & 0.00 & 0.02 & .08 & .880 \\
\hline BFNE & 0.00 & 0.03 & .09 & .931 & 0.01 & 0.02 & .11 & .679 & 0.02 & 0.02 & .43 & .267 \\
\hline SAAS & 0.03 & 0.02 & 1.46 & .159 & 0.07 & 0.02 & 1.17 & $.001 *$ & -0.00 & 0.02 & -.06 & .922 \\
\hline MEC & 0.01 & 0.02 & .73 & .473 & -0.01 & 0.01 & -.31 & .424 & 0.00 & 0.01 & .24 & .682 \\
\hline SOP & -0.02 & 0.03 & -.59 & .562 & 0.02 & 0.02 & .37 & .331 & 0.00 & 0.02 & .01 & .991 \\
\hline WAI & 0.01 & 0.04 & .22 & .830 & -0.06 & 0.03 & -.47 & .065 & -0.02 & 0.02 & -.34 & .341 \\
\hline$R^{2}$ & & .193 & & & & .408 & & & & .267 & & \\
\hline
\end{tabular}

Note. $\mathrm{CO}_{2}=$ carbon dioxide; $\mathrm{BN}=$ Bulimia Nervosa; VAS = visual analogue scale; PSL = Panic Symptoms List $; \mathrm{HR}=$ heart rate $;$ SPIN = Social Phobia Inventory; BFNE = Brief Fear of Negative Evaluation Scale; SAAS = Social Appearance Anxiety Scale; MEC = Maladaptive evaluative concerns; SOP = Self-oriented perfectionism; WAI = Worry about imperfection.

Note. $N=56$ for the prediction of VAS-fear and PSL scores; $N=21$ for the prediction of HR.

Note. NED group is coded as $0 ; \mathrm{BN}$ group is coded as 1 .

* indicates a significant predictor variable. 
Table 19

Regression Analysis Predicting Panic Symptoms in Response to $\mathrm{CO}_{2}$ by Social Anxiety Symptoms and Social Anxiety-Related Variables, in the NED Group

\begin{tabular}{|c|c|c|c|c|}
\hline & \multicolumn{4}{|c|}{ Predicting PSL scores } \\
\hline & $B$ & $S E B$ & $\beta$ & $P$ \\
\hline \multicolumn{5}{|l|}{ NED Group } \\
\hline \multicolumn{5}{|l|}{ Step 1} \\
\hline SPIN & 0.01 & 0.01 & .22 & .260 \\
\hline$R^{2}$ & & .049 & & \\
\hline \multicolumn{5}{|l|}{ Step 2} \\
\hline SPIN & 0.01 & 0.02 & .15 & .562 \\
\hline BFNE & -0.03 & 0.02 & -.41 & .175 \\
\hline SAAS & 0.03 & 0.03 & .36 & .331 \\
\hline MEC & 0.01 & 0.01 & .23 & .581 \\
\hline SOP & 0.01 & 0.01 & .36 & .337 \\
\hline WAI & -0.02 & 0.03 & -.24 & .467 \\
\hline$R^{2}$ & & .310 & & \\
\hline
\end{tabular}

Note. $\mathrm{CO}_{2}=$ carbon dioxide $\mathrm{NED}=$ non-eating disorder; $\mathrm{VAS}=$ visual analogue scale; PSL = Panic Symptoms List $;$ SPIN = Social Phobia Inventory $;$ BFNE = Brief Fear of Negative Evaluation Scale; SAAS = Social Appearance Anxiety Scale; MEC = Maladaptive evaluative concerns; $\mathrm{SOP}=$ Self-oriented perfectionism; $\mathrm{WAI}=$ Worry about imperfection. Note. NED group is coded as $0 ; \mathrm{BN}$ group is coded as 1 .

Note. $N=56$. 
Table 20

Regression Analysis Predicting DIA-BP in Response to the BIS by Social Anxiety Symptoms and Social Anxiety-Related Variables, in the BN Group

\begin{tabular}{|c|c|c|c|c|}
\hline \multicolumn{5}{|c|}{ Predicting DIA-BP } \\
\hline & $B$ & $S E B$ & $\beta$ & $P$ \\
\hline \multicolumn{5}{|l|}{ BN Group } \\
\hline \multicolumn{5}{|l|}{ Step 1} \\
\hline SPIN & 0.01 & 0.01 & .18 & .448 \\
\hline$R^{2}$ & & .031 & & \\
\hline \multicolumn{5}{|l|}{ Step 2} \\
\hline SPIN & -0.01 & 0.02 & -.26 & .590 \\
\hline BFNE & 0.02 & 0.01 & .58 & .102 \\
\hline SAAS & 0.01 & 0.01 & .31 & .586 \\
\hline MEC & -0.01 & 0.01 & -.60 & .272 \\
\hline SOP & 0.01 & 0.01 & .81 & .130 \\
\hline WAI & -0.03 & 0.02 & -.55 & .097 \\
\hline$R^{2}$ & & .411 & & \\
\hline \multicolumn{5}{|c|}{$\begin{array}{l}\text { Note. } \text { BIS = body image stressor; } \mathrm{BN}=\text { Bulimia Nervosa; DIA-BP = diastolic } \\
\text { blood pressure; SPIN = Social Phobia Inventory; } \mathrm{BFNE}=\text { Brief Fear of Negative } \\
\text { Evaluation Scale; SAAS = Social Appearance Anxiety Scale; } \mathrm{MEC}=\text { Maladaptive } \\
\text { evaluative concerns; SOP = Self-oriented perfectionism; WAI = Worry about } \\
\text { imperfection. } \\
\text { Note. NED group is coded as } 0 ; \mathrm{BN} \text { group is coded as } 1 . \\
\text { Note. } N=21 .\end{array}$} \\
\hline
\end{tabular}


Table 21

Regression Analysis Predicting Anxiety and DIA-BP in Response to the BIS by Social Anxiety Symptoms and Social Anxiety-Related Variables, in the NED Group

\begin{tabular}{ccccccccc}
\hline & \multicolumn{3}{c}{ Predicting VAS-Anxiety } & \multicolumn{5}{c}{ Predicting DIA-BP } \\
\hline NED Group & $B$ & SE B & $\beta$ & $P$ & $B$ & $S E B$ & $\beta$ & $P$ \\
\hline Step 1 & & & & & & & & \\
SPIN & -0.01 & 0.01 & -.17 & .390 & -0.01 & 0.01 & -.28 & .190 \\
$R^{2}$ & & .029 & & & & .080 & & \\
Step 2 & & & & & & & & \\
SPIN & -0.01 & 0.01 & -.32 & .264 & -0.02 & 0.01 & -.48 & .152 \\
BFNE & -0.01 & 0.01 & -.25 & .438 & -0.01 & 0.01 & -.19 & .608 \\
SAAS & 0.01 & 0.02 & .17 & .678 & 0.02 & 0.02 & .31 & .498 \\
MEC & 0.00 & 0.01 & .28 & .540 & 0.01 & 0.01 & .48 & .385 \\
SOP & 0.00 & 0.01 & .09 & .816 & -0.01 & 0.01 & -.26 & .590 \\
WAI & 0.01 & 0.02 & .10 & .778 & 0.00 & 0.02 & -.01 & .983 \\
$R^{2}$ & & .170 & & & & & .189 & \\
\hline
\end{tabular}

Note. BIS = body image stressor; NED = non-eating disorder; VAS = visual analogue scale; DIA-BP = diastolic blood pressure SPIN = Social Phobia Inventory; BFNE = Brief Fear of Negative Evaluation Scale; SAAS = Social Appearance Anxiety Scale; $\mathrm{MEC}=$ Maladaptive evaluative concerns; $\mathrm{SOP}=$ Self-oriented perfectionism; $\mathrm{WAI}=$ Worry about imperfection .

Note. $N=28$ for the prediction of VAS-Anxiety; $N=23$ for the prediction of DIA-BP.

Note. NED group is coded as $0 ; \mathrm{BN}$ group is coded as 1 . 
Hypothesis 3: Associations Among Stressor Response and Psychological Symptoms (Social Anxiety, Depression, Trait anxiety) that Commonly Co-occur with BN.

For each stressor task, within the $\mathrm{BN}$ group only, correlational analyses were performed among all stress response indicators (both subjective and objective) and total scores on three symptom measures: SPIN, DASS-21-D, and STICSA-Trait. Tables 22-24 display all Pearson product-moment correlation coefficients. Findings demonstrated that higher SPIN scores were correlated with higher VAS ratings of body dissatisfaction. Neither DASS-21-D nor STICSATrait scores were significantly correlated with subjective or physiological stress response indicators.

\section{Hypothesis 4: Associations Between Stress Reactivity (Anxiety, Fear, Stress, Panic Symptoms) and Body Image/Eating Specific Responses (Desire to Eat, Hunger, Body Dissatisfaction) in BN.}

For each stressor task, within the BN group only, correlational analyses among the seven subjective stress response indicators were examined. These Pearson product-moment correlation coefficients are also displayed in Tables 22-24. Analyses revealed two significant correlations. Specifically, in response to the $\mathrm{CO}_{2}$ task, greater levels of anxiety were correlated with lower levels of body dissatisfaction $(r=-.40, p=.04)$. Additionally, in response to the TSST, greater levels of fear were correlated with greater levels of body dissatisfaction $(r=.42, p=.03)$. No other significant correlations were revealed. Given multiple comparisons, a Bonferroni correction was then applied to the data to maintain the familywise error rate. With this new alpha in place $(p<.006)$, the aforementioned findings were no longer significant. 


\section{Hypothesis 5: Differential Stressor Effects In Individuals With BN.}

To address the final objective of this dissertation, differential stressor effects within the BN group were investigated. A repeated measures ANOVA was performed to compare, within participants, change (from pre to poststressor) among the three distinct stressor tasks. When the assumption of sphericity was violated, a Greenhouse-Geisser correction was applied to the degrees of freedom and $F$ test statistic. Findings are displayed in Table 25; no significant withinparticipant effects of three stressor tasks were revealed. The second part of this research question intended to investigate social anxiety related factors (if any) that differentiate those who reacted most to one stressor compared to another. This question could not be addressed given the null results of the initial analysis. 
Table 22

Correlations (r) Among Responses to the TSST, the DASS-21-D and the STICSA-Trait, in the BN group

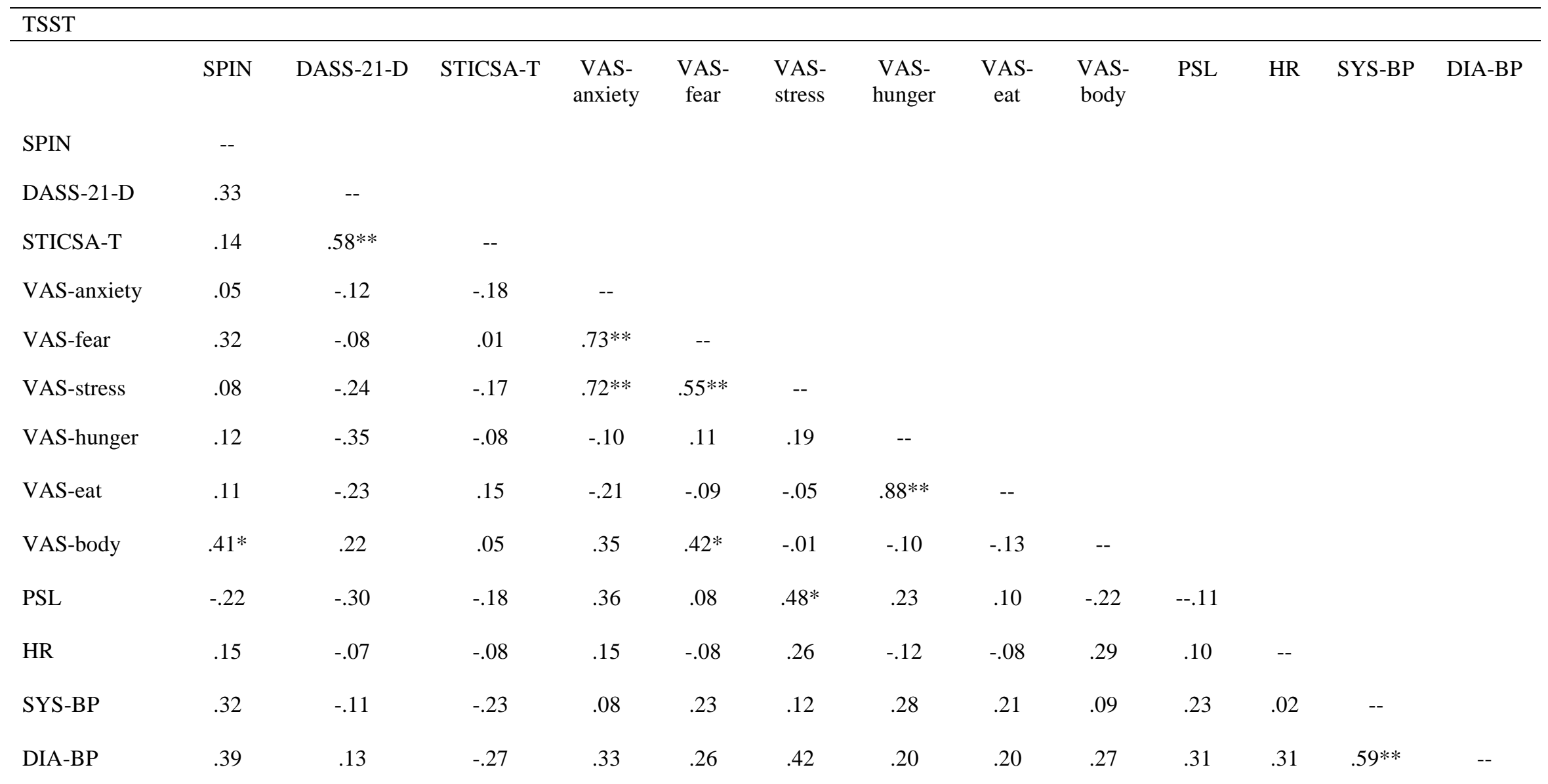

Note. DASS-21-D = Depression Anxiety Stress Scales - 21 item version, depression subscale; STICSA = State Trait Inventory for Cognitive and Somatic

Anxiety; $\mathrm{BN}=$ Bulimia Nervosa; $\mathrm{CO}_{2}=$ carbon dioxide; $\mathrm{SPIN}=$ Social Phobia Inventory; VAS = visual analogue scale; PSL = Panic Symptom List $; \mathrm{HR}=$ heart rate; $\mathrm{BP}=$ blood pressure; $\mathrm{SYS}=$ systolic; DIA = diastolic .

Note. $N=21$ for physiological data; $N=28$ for subjective data. $* * p<0.01$ (2-tailed); $* p<0.05$ (2-tailed) 
Table 23

Correlations (r) Among Responses to $\mathrm{CO}_{2}$, the DASS-21-D and the STICSA-T, in the BN group

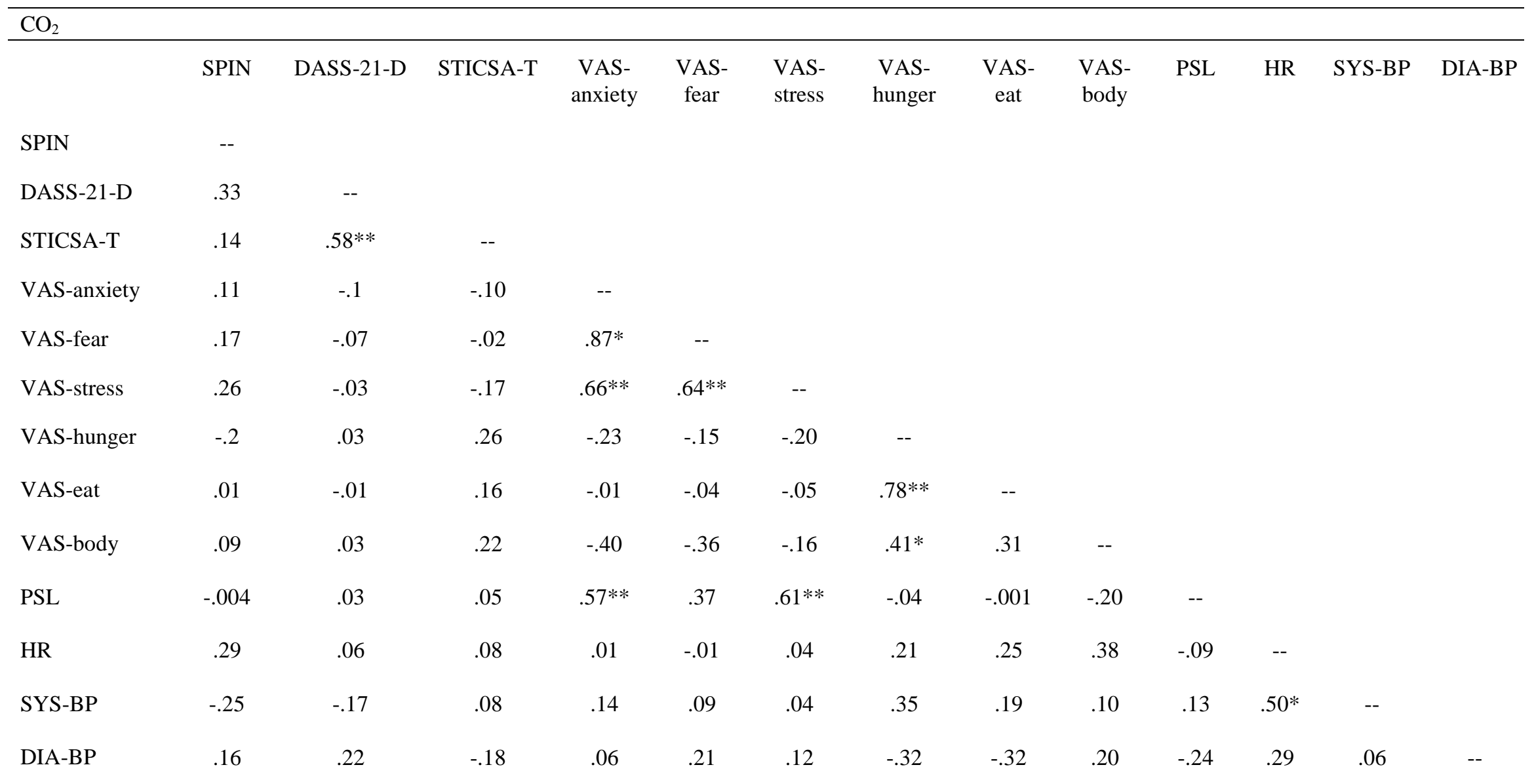

Note. DASS-21-D = Depression Anxiety Stress Scales - 21 item version, depression subscale; STICSA = State Trait Inventory for Cognitive and Somatic Anxiety; $\mathrm{BN}=$ Bulimia Nervosa; $\mathrm{CO}_{2}=$ carbon dioxide; $\mathrm{SPIN}=$ Social Phobia Inventory; VAS = visual analogue scale; PSL = Panic Symptom List; HR = heart rate; $\mathrm{BP}=$ blood pressure; $\mathrm{SYS}=$ systolic; DIA = diastolic.

Note. $N=21$ for physiological data; $N=28$ for subjective data. $* * p<0.01$ ( 2 -tailed); $* p<0.05$ (2-tailed) 
Table 24

Correlations (r) Among Responses to the BIS, the DASS-21-D and the STICSA-T, in the BN group

\begin{tabular}{|c|c|c|c|c|c|c|c|c|c|c|c|c|c|}
\hline \multicolumn{14}{|l|}{ BIS } \\
\hline & SPIN & DASS-21-D & STICSA-T & $\begin{array}{l}\text { VAS- } \\
\text { anxiety }\end{array}$ & $\begin{array}{l}\text { VAS- } \\
\text { fear }\end{array}$ & $\begin{array}{l}\text { VAS- } \\
\text { stress }\end{array}$ & $\begin{array}{l}\text { VAS- } \\
\text { hunger }\end{array}$ & $\begin{array}{l}\text { VAS- } \\
\text { eat }\end{array}$ & $\begin{array}{l}\text { VAS- } \\
\text { body }\end{array}$ & PSL & HR & SYS-BP & DIA-BP \\
\hline DASS-21-D & .33 & -- & & & & & & & & & & & \\
\hline STICSA-T & .14 & $.58 * *$ & -- & & & & & & & & & & \\
\hline VAS-fear & .35 & -.15 & .10 & $.47^{*}$ & -- & & & & & & & & \\
\hline VAS-stress & .32 & -.01 & .20 & $.64^{* *}$ & $.59 * *$ & -- & & & & & & & \\
\hline VAS-hunger & -.15 & -.04 & .30 & -.11 & .06 & .17 & -- & & & & & & \\
\hline VAS-eat & -.20 & -.27 & -.15 & .01 & .08 & .25 & .22 & -- & & & & & \\
\hline HR & .21 & -.28 & -.24 & .10 & .22 & .14 & -.19 & -.07 & .04 & -.01 & -- & & \\
\hline SYS-BP & .18 & .39 & .28 & -.05 & -.04 & -.03 & -.21 & -.38 & .22 & -.04 & -.12 & -- & \\
\hline DIA-BP & .18 & .02 & .04 & .11 & .09 & .002 & -.01 & -.34 & .17 & -.01 & .27 & $.73 * *$ & -- \\
\hline
\end{tabular}

Note. DASS-21-D = Depression Anxiety Stress Scales - 21 item version, depression subscale; STICSA = State Trait Inventory for Cognitive and Somatic Anxiety; $\mathrm{BN}=$ Bulimia Nervosa; $\mathrm{CO}_{2}=$ carbon dioxide; $\mathrm{SPIN}=$ Social Phobia Inventory; VAS = visual analogue scale; PSL = Panic Symptom List; HR = heart rate; $\mathrm{BP}=$ blood pressure; $\mathrm{SYS}=$ systolic; $\mathrm{DIA}=$ diastolic

Note. $N=21$ for physiological data; $N=28$ for subjective data. $* * p<0.01$ (2-tailed); $* p<0.05$ (2-tailed) 
Table 25

Repeated Measures Analysis of Variance of Within-Participant Effects of Three Stressor Tasks (Change from Time 1 to Time 2), in the BN Group

\begin{tabular}{lcccc} 
& \multicolumn{3}{c}{ Within effect (stressor task) } \\
\cline { 2 - 5 } & $d f$ & $F$ & $P$ & $\eta^{2}$ \\
\hline VAS-anxiety & 2 & 0.13 & .882 & .005 \\
VAS-fear & 1.58 & 0.02 & .978 & .001 \\
VAS-stress & 2 & 0.35 & .708 & .013 \\
VAS-hunger & 1.35 & 2.34 & .126 & .080 \\
VAS- eat & 2 & 0.91 & .410 & .032 \\
VAS-body & 2 & 0.63 & .534 & .023 \\
PSL & 2 & 0.10 & .908 & .004 \\
\hline
\end{tabular}

Note. VAS = visual analogue scale; PSL = Panic Symptoms List . 


\section{Discussion}

The purpose of this dissertation was to advance the study of stress response in individuals with BN. This clinical group is generally well known to respond negatively to stress, as supported by prominent theories of binge eating development and maintenance (Fairburn, 1997; Hawkins \& Clement, 1984; Polivy \& Herman, 1985). However, studies have not adequately considered how common comorbid conditions, such as social anxiety, affect stress response in this population. In addition, while the influence of perceived interpersonal stress (as one specific type of stress) on $\mathrm{BN}$ symptoms has been documented, this association has not been investigated in individuals with highs levels of social anxiety who are evidently more likely to experience interpersonal stress. It is important to understand the consequences of this specific relationship, in addition to the relationship between $\mathrm{BN}$ and other types of stress and the potential influence of social anxiety symptoms.

Accordingly, the current study investigated the responses of individuals with BN compared to individuals without an eating disorder to three distinct laboratory stressors: a psychosocial stressor, a physiological stressor, and an eating disorder specific (body image) stressor. Commonly known factors that affect stress response were considered. To contribute to the growing body of research investigating social anxiety in $\mathrm{BN}$, social anxiety symptoms and social anxiety related factors were then examined as possible predictors of stress response in each group. Subsequently, in the BN group alone, the ability of other common comorbid conditions to predict response to stressors was evaluated. Also in the BN group alone, the possible link between stress reactivity (anxiety, fear, stress, panic symptoms) and eating/body image-related response (body dissatisfaction, desire to eat, hunger) was evaluated. Lastly, 
within-participant effects of the three laboratory stressors were explored in the BN group, for the purpose of contributing methodologically to the study of stress in BN.

Stress reactivity was defined by both psychological variables (subjective responses to self-report questionnaires) and physiological indices (cardiovascular response; heart rate, systolic blood pressure, diastolic blood pressure). Eating/body image related response was defined by subjective responses to a self-report questionnaire. To note, effect sizes associated with all findings in the present study were interpreted according to Ferguson's (2009) effect size guidelines for social science data. Specifically, a Cohen's $d$ value with a magnitude between .41 and 1.15 is considered the minimum effect size representing a practically significant effect; a beta value of magnitude between .2 and .5 is considered the minimum effect size representing a practically significant effect. In the discussion below, an effect size reported as small indicates one within the above-mentioned range (unless otherwise indicated). An effect size reported as trivial indicates one below the above-mentioned range (i.e., not practically significant).

\section{Manipulation Checks}

\section{Within-subjects change in subjective stress response indicators, across groups. First,}

it was necessary to determine whether the stressors utilized in this study indeed provoked change in stress response indicators from time 1 to time 2, on average, across all participants (regardless of group allocation). Ratings of various sensations (e.g., anxiety) on VAS are often used in stress research as measures of subjective reactivity to stressful stimuli. As a manipulation check for each stressor task, within-subjects change in VAS ratings of anxiety, fear, and stress, as well as PSL scores of panic symptoms, was investigated.

Results demonstrated that the TSST provoked significant increases in all four indicators of subjective stress reactivity, with small effects for anxiety, stress, and panic symptoms, and a 
trivial effect size for fear. The $\mathrm{CO}_{2}$ stressor provoked significant increases in fear and panic symptoms, with small effect sizes. Anxiety and stress levels did not change in response to $\mathrm{CO}_{2}$, and the associated effect sizes were trivial. Asking participants about their level of stress as a response indicator is not typical in stress research. Given the momentary nature of the $\mathrm{CO}_{2}$ stress response, stress as a cognitive state of emotional tension might not have been perceived during such a short time period.

Overall, the abovementioned findings were consistent with hypotheses and general expectations based on previous studies that used VAS and panic symptom measures to assess reactivity to the TSST and $\mathrm{CO}_{2}$ (e.g., Campbell \& Ehlert, 2012; Kaye, Buchanan et al., 2004). The BIS, on the other hand, did not provoke a significant change in anxiety, fear, stress, or panic symptoms across the entire study sample, and effect sizes were trivial. This was not surprising, however, given that the BIS was developed as an eating disorder specific stressor and thus was expected to exert effects only within the BN group and not across all participants in the sample.

\section{Within-subjects change in physiological stress response indicators, across groups.}

Within-subjects change in physiological measures of stress reactivity (HR, SYS-BP, DIA-BP) from time 1 to time 2 was also investigated across all participants as a manipulation check. Consistent with expectations, results demonstrated that the TSST provoked increased HR and DIA-BP with small effect sizes. The $\mathrm{CO}_{2}$ stressor also provoked increased HR with a small effect size. No other significant physiological changes were revealed during the TSST or $\mathrm{CO}_{2}$ and effect sizes of insignificant findings were trivial, which were inconsistent with expectations. The BIS provoked increased DIA-BP with a small effect size. Significant changes in HR and SYS-BP were not revealed, and again the effect sizes related to these statistically nonsignificant findings were trivial. 
As noted above, it was expected that the BIS would significantly affect only individuals with $\mathrm{BN}$; moreover, since this was the first study to investigate physiological response to the BIS, change from time 1 to time 2 could not be expected as it was for the other two stressor tasks. Certainly, when investigating physiological indices of stress reactivity, consideration must be given to participants' current health and medical history. Half the sample in this study consisted of individuals with a diagnosis of $\mathrm{BN}$, and some studies have demonstrated a dysregulated stress response system in this population (e.g., Koo-Loeb, 1998; Pirke, Platte, Laessle, Seidl, \& Fichter, 1992).

More generally, consistent with the abovementioned findings, it is well recognized that ratings of subjective stress are not necessarily highly correlated with physiological stress symptoms (Allen et al., 2014). Given that the three stressors utilized in this study each brought about some increase in at least one type of stress symptom from time 1 to time 2 , the manipulation check was supported and the use of these stressors with the present study sample was tentatively validated.

\section{Did participants' cardiovascular levels return to baseline following each task? Given} that participants underwent the three stressors in a single study session, it was necessary to ensure that they recovered from the effects of each stressor prior to proceeding with the next study task (as demonstrated in previous research that utilized multiple stressor tasks in a single session; e.g., Allen et al., 2002). Consistent with predictions, repeated measures ANOVAs (across all participants) revealed that mean HR and BP values during the recovery period following each stressor were either comparable to or lower than HR and BP values during a baseline rest period. These findings provided fundamental support for the current study's methodology, and analyses to address the main study objectives were next conducted. 


\section{Group specific within-subjects change in subjective response indicators. To test}

certain study hypotheses, it was necessary to first examine whether the stressors indeed provoked change in response indicators (both stress reactivity and body image/eating related responses) within the specific study groups. Therefore, within-groups analyses of within-subject change in response indicators from time 1 to time 2 were performed to provide greater insight into groupspecific effects of the stressors.

\section{BN group - within-subjects change in subjective response indicators from time 1 to}

time 2. Overall in the $\mathrm{BN}$ group, there was no consistent pattern of change in response indicators across the three stressors.

The TSST provoked significant increases in all four indicators of stress reactivity with small effect sizes, consistent with hypotheses. However, the TSST did not provoke significant change in any of the body image/eating specific responses (VAS ratings of body dissatisfaction, hunger, desire to eat), and the strength of these effects was trivial.

The $\mathrm{CO}_{2}$ stressor provoked significant increases in fear and panic symptoms with small effect sizes, consistent with hypotheses. No significant changes in anxiety or stress were observed and the strength of these effects was trivial. The BN group demonstrated significant decreases in hunger and desire to eat with small effect sizes, which was inconsistent with hypotheses. No significant changes in body dissatisfaction were revealed and the effect size was trivial.

For the BIS, inconsistent with hypotheses, no significant changes in any indicators of stress reactivity were observed and effect sizes were trivial, although change was in the expected direction. Pilot data of the BIS (as shown in Appendix A) demonstrated that increased anxiety from time 1 to time 2 , although not statistically significant, was associated with a small effect. 
Accordingly, an increase in anxiety within-participants from time 1 to time 2 was expected in the current dissertation given its larger sample size, yet results were inconsistent with this expectation. In terms of body image/eating specific responses, the BIS provoked significant increases in body dissatisfaction (consistent with hypotheses) and decreases in hunger (inconsistent with hypotheses); both findings were associated with small effect sizes. The BIS did not provoke change in desire to eat (inconsistent with hypotheses), and the associated effect size was trivial.

\section{NED group - within-subjects change in subjective response indicators from time 1 to}

time 2. Overall, in the NED group, there was no consistent pattern of change in response indicators across the three stressors.

The TSST provoked significant increases in anxiety, stress, and panic symptoms with small effect sizes, consistent with hypotheses. However, the TSST did not provoke significant change in fear (and the effect size was small), although change was in the expected direction. Also inconsistent with hypotheses, significant decreases were observed in ratings of body dissatisfaction and hunger, with small effect sizes. Change in desire to eat was not significant and the effect size was trivial.

The $\mathrm{CO}_{2}$ stressor provoked significant increases in panic symptoms with a small effect size, consistent with hypotheses. No significant changes in anxiety, fear or stress were observed, and effect sizes were trivial, inconsistent with hypotheses. Also inconsistent with hypotheses, significant decreases in body dissatisfaction and desire to eat were observed with small effect sizes. Hunger ratings did not change significantly, and the associated effect size was trivial in magnitude. 
The BIS provoked only one significant change: anxiety decreased from time 1 to time 2 , consistent with pilot study findings. No other significant changes were observed, and effect sizes were generally trivial in magnitude. It is interesting to note that all subjective response indicators (except for body dissatisfaction) decreased from time 1 to time 2, indicating that individuals in the NED group felt less anxious, fearful, stressed, and panicked following the BIS. Of course, one cannot expect body image concerns to be completely absent in normal eaters, and thus it makes sense that change in body dissatisfaction from time 1 to time 2 moved in the same direction as that in the $\mathrm{BN}$ group.

Within-subjects change in physiological response indicators. In terms of physiological change provoked by the stressors, few findings were consistent with hypotheses, and there was no pattern of consistent increases or decreases in HR or BP values. In response to the TSST, HR significantly increased with small and moderate (Ferguson, 2009) effect sizes in both the BN and NED groups, respectively. DIA-BP also increased in each group with small effect sizes. In response to $\mathrm{CO}_{2}$, $\mathrm{HR}$ increased significantly with a small effect size in the $\mathrm{BN}$ group. No significant changes were observed in the NED group. In response to the BIS, DIA-BP significantly decreased with small effect sizes in both groups.

\section{Hypothesis Testing}

Group comparisons of subjective response to stressors. Between-group differences in subjective response to the stressors were examined. For the TSST, increase in panic symptoms from time 1 to time 2 was significantly greater in the BN group compared to the NED group, with a small effect size. This was consistent with prediction. The remaining TSST responses were not significantly different between groups; however, small effect sizes were revealed for change in fear, stress and hunger. No significant differences between groups were found in 
response to $\mathrm{CO}_{2}$, inconsistent with hypotheses, and only fear was associated with a small effect size. In response to the BIS, increases in anxiety, body dissatisfaction, and stress were significantly greater in the BN group compared to the NED group, consistent with prediction. Although fear responses were not significantly different between groups, a small effect size was revealed. No other significant between group differences were found, and only panic symptoms were associated with a small effect size.

In summary, for the most part, findings in this sample demonstrated statistically nonsignificant between group differences in subjective stressor response. Nonetheless, hypotheses were somewhat supported given the above-mentioned estimates of effect size for specific response indicators, which may suggest that this study lacked sufficient power to detect significant effects. Moreover, although some findings were inconsistent with hypotheses, divergence in the components of stress response is not uncommon (Lazarus, 1993).

Upon a visual inspection of mean response ratings in each study group (as depicted in Table 3), it was evident that individuals in the BN group had higher initial (time 1) VAS ratings and PSL scores compared to the NED group, for each stressor. In addition, at time 2, the BN group consistently rated each subjective stress response indicator at a higher level than the NED group, for each stressor. For the most part, responses in the BN group increased in a linear fashion alongside those in the NED group; this explains the insignificant between group differences in change from time 1 to time 2 . This finding is similar to a recent study that demonstrated more negative affect overall in an eating disorder group, although no difference in affective stressor response compared to a healthy control group (Het, Wolf, Hammelstein, Herpertz, \& Wolf, 2015). 
Further speculation can be made regarding the present study's findings by determining if they were anomalous or consistent with previous studies of the same stressor tasks. Along these lines, with respect to the $\mathrm{CO}_{2}$ stressor, VAS anxiety ratings in the present study were compared to those found in a previous study (Woznica et al., 2015) that examined panic reactivity to $\mathrm{CO}_{2}$ in individuals with BN. Woznica and colleagues (2015) found that individuals with BN demonstrated greater increases in VAS anxiety ratings from pre to post- $\mathrm{CO}_{2}$ compared to a control group, with an effect size of $d=.73$. Not only did the present study find nonsignificant group differences in VAS anxiety ratings, but also the size of this effect was much smaller than that in the previous study $(d=.23)$. In part, an explanation for this discrepancy might be that the dispersions of VAS anxiety ratings within each group (i.e., variance within the BN group and variance within the NED group) were greater in the present study, at each measurement point, compared to the variance estimates of the respective groups in the Woznica et al. (2015) study.

One possibility for why this greater variability exists concerns differences in sample characteristics between the present study and Woznica et al. (2015), which might have contributed to dissimilar study findings. The BN group in Woznica et al. (2015) averaged 5.4 ( $\mathrm{SD}=4.6)$ binges and $4.8(\mathrm{SD}=5.0)$ purges per week over the previous 3 months. In contrast, the frequency of bingeing and purging was much lower in the present study; participants reported an average of $2.36(S D=1.87)$ objective binges and $2.96(S D=2.99)$ compensatory behaviours per week over the previous 3 months. Also, approximately one fifth of the present study sample engaged in nonpurging (dietary restriction, excessive exercise) compensatory behaviours. In Woznica et al. (2015), only females with purging behaviours (self-induced vomiting, laxative misuse) were eligible to participate. 
Eligibility criteria for the BN group in the present study reflected the change from DSMIV to DSM-5 diagnostic criteria for BN; specifically, the subtyping of this diagnosis into purging and nonpurging forms was replaced by a single diagnosis that includes the use of both purging and nonpurging weight-control behaviours. The reason for this change was twofold: (1) little empirical evidence exists to support that $\mathrm{BN}$ characterized by purging behaviours are more severe than those involving nonpurging behaviours; and (2) nonpurging behaviours are observed with sufficient frequency to merit their continued inclusion in diagnostic criteria for the disorder (Mond, Hay, Rodgers, Owen, Crosby, \& Mitchell, 2006; Mond, Hay, Rodgers, Owen, \& Mitchell, 2006; Mond, Rodgers, Hay, \& Owen, 2011; Wilfley, Bishop, Wilson, \& Agras, 2007; van Hoeken, Veling, Sinke, Mitchell, Hoek, 2009). However, the fact remains that most research on BN has focused on individuals who actively purge via self-induced vomiting, including the previous $\mathrm{CO}_{2}$ study; and therefore, direct comparisons between participant samples cannot be made.

One additional study looked at $\mathrm{CO}_{2}$ response in a mixed ED sample composed of individuals with either AN or BN compared to healthy controls. VAS anxiety ratings were used in this study as one measure of stress response (Perna et al., 2004), and similar responses were demonstrated in both study groups, consistent with the present study. Also of note is that the methodology in both aforementioned studies (Perna et al, 2004; Woznica et al., 2015) was somewhat different from that in the current study. More specifically, in panic research, response to $\mathrm{CO}_{2}$ inhalation is typically compared with response to a room air inhalation (placebo versus active agent) rather than to a baseline measure (prestressor versus poststressor). The present study's methodology followed what is commonly used in the $\mathrm{CO}_{2}$ stress literature (e.g., Kaye, Buchanan, et al., 2004) as opposed to the $\mathrm{CO}_{2}$ panic literature. Arguably, a placebo condition is 
not essential to the task's procedure as participants are able to familiarize themselves with the breathing apparatus during a baseline practice period, thus reducing potential anticipatory anxiety during the subsequent $\mathrm{CO}_{2}$ breath derived from the breathing apparatus itself. In this sense, utilizing a placebo condition (versus a baseline measure) might actually be a more stringent comparison. To explain, between-group differences might be less likely when comparing response of $\mathrm{a} \mathrm{CO}_{2}$ inhalation to a placebo inhalation versus a baseline measure if anxiety related to the breathing apparatus is still present during the $\mathrm{CO}_{2}$ inhalation. If this were the case, between-group differences in the present study (which compared $\mathrm{CO}_{2}$ response to a baseline measure) would be more likely; however, this expectation was not supported by study findings. Therefore, it is possible that this methodological feature did not contribute to the different findings in these two studies.

Few studies have used the TSST (or modified versions of the TSST) as a stressor task in BN samples (e.g., Ginty et al., 2012; Het et al., 2015; Monteleone et al., 2012). The most recent study in a mixed ED group compared to a healthy control group measured negative affect as a psychological response to the TSST via the Positive and Negative Affect Schedule (Watson et al., 1988). Results revealed that while ED is associated with elevated negative affect, no betweengroup differences were found (effect sizes were not reported). This specific response to interpersonal stress has been demonstrated in numerous other studies (e.g., Hilbert, Vogele, Tuschen-Caffier, \& Hartmann, 2011; Pirke et al., 1992; Vocks, Legenbauer, Wachter, Wucherer, \& Kosfelder, 2007); yet negative affect is a general factor of subjective distress, and subsumes a broad range of negative mood states (including, for example, fear, anxiety, hostility; Watson et al., 1988). Therefore, these findings cannot be directly comparable to the response indicators 
measured in the present study. Subjective effects of the TSST, as measured in the present study, were unfortunately not emphasized in the other previous studies of eating disordered samples.

However, speculation can be made regarding the single significant between-group difference found in response to the TSST (i.e., greater increase in panic symptoms in the BN group compared to the NED group, with a medium effect size). Group was no longer a predictor of change in panic symptom scores from time 1 to time 2 once other possible predictors were entered into a regression model. Rather, DASS-21-D scores and PSS scores significantly predicted change in panic symptom scores. This is consistent with research that has demonstrated a moderating effect of stress appraisal on stress response (Lazarus \& Folkman, 1984). The PSS was used in the present study to target general stress perception, and individuals in the BN group scored higher on this scale than individuals in the NED group, indicating greater levels of perceived stress. Although inconsistent with research demonstrating effects of depression on TSST response (e.g., Chopra et al., 2009; Dienes, Hazel, \& Hammen, 2013), it should be considered that panic symptom scores were not assessed in these studies. Responses to this particular set of symptoms might not be generalizable to the abovementioned previous study findings. Individuals in the BN group also scored higher on the DASS-21-D than individuals in the NED group, indicating greater levels of depressive symptoms; individuals with depression do not generally react with enhanced panic symptoms in response to $\mathrm{CO}_{2}$ (Perna et al., 1995); perhaps this finding can help to explain that in the present study.

The aforementioned results suggest that a diagnosis of BN per se does not account for the greater increase in panic symptoms after the TSST in BN compared to NED, and implicates stress appraisal and depressed mood as influences on the relationship between $\mathrm{BN}$ and stress response. Other potential factors might have also contributed to why the BN group demonstrated 
significantly greater change compared to the NED group in panic symptoms only, and not in any other subjective response indicators. Panic symptoms were rated on the PSL, a self-report questionnaire with a Likert scale to denote responses, rather than a continuous visual analogue scale that was used to rate all other psychological outcome variables in this study. Perhaps the PSL was simply more sensitive in detecting changes given the specificity of symptoms that are assessed on this scale, rather than inquiring more generally about a feeling of anxiety, fear, or stress. Perhaps participants in the BN group were more attuned to their bodily sensations rather than their cognitions.

Another consideration is that the two groups differed in their history of unexpected panic attacks, with $28 \%$ of individuals in the BN group compared to $3.6 \%$ of individuals in the NED group endorsing the experience of at least two unexpected panic attacks in their lifetime. History of unexpected panic attacks is an established predictor of greater response to $\mathrm{CO}_{2}$ (Perna et al., 1995); it was not an exclusion criterion for participants in the current study to ensure it was feasible to obtain a large enough sample, particularly in the BN group. With respect to the TSST specifically, one study demonstrated a trend toward greater poststressor subjective anxiety in those with panic disorder compared to healthy controls (Petrowski, Winterman, Shaarschmidt, Bornstein, \& Kirschbaum, 2013). Although no participants in the current sample endorsed diagnostic criteria for panic disorder, perhaps the presence of unexpected panic attacks alone is a significant contributor to the present study result. An additional between group difference in the present study was that individuals in the BN group had significantly greater ASI-3 scores compared to the NED group; elevated anxiety sensitivity is associated with a propensity to panic (McNally, 2002); however, because ASI-3 scores did not significantly predict response to the TSST, this group difference in ASI-3 scores is an unlikely explanation for the present finding. 
A closer look was taken at social anxiety rates within each group, in an attempt to shed further light on the null TSST between-group findings (aside from PSL scores). As previously reported in the Results (Table 2), the proportions of SAD diagnoses within each group were significantly different from one another; $35.7 \%$ of individuals in the $\mathrm{BN}$ group endorsed diagnostic criteria for SAD compared to $7.1 \%$ of individuals in the NED group. In the BN group, SPIN scores averaged within the clinical range, but in the NED group SPIN scores averaged well below the suggested cut score of 19 for clinically relevant symptoms (Conners et al., 2000). Given the higher levels of social anxiety symptoms among those in the BN group, one might expect this group to have a stronger reaction to the TSST, an interpersonal stressor, compared to the NED group (if taking into account previous TSST studies showing elevated response in individuals with SAD; e.g., Krämer et al., 2012). However, as mentioned, social anxiety did not predict TSST response in the present study. One possibility to consider is that SPIN scores were not an appropriate proxy for SAD in the context of this study.

Following pilot testing (Linett et al., 2016; see Appendix B), the BIS was used in the present study to assess the effects of an eating disorder specific stressor that is devoid of obvious social influences. Specifically, participants underwent the stressor alone in a testing room, and no people or bodies were shown in the slideshow images of clothing articles. This stressor was meant to particularly affect individuals with disordered eating via its effect on body image distress. Consistent with hypotheses, increases in anxiety, body dissatisfaction, and stress ratings from pre- to post-BIS were significantly greater in the BN group compared to the NED group. ASI-3 scores, however, were also found to predict change in stress ratings (and ASI-3 as a predictor of change in anxiety ratings was marginally significant). When multiple variables were entered into a regression model, group remained a unique predictor of change in anxiety ratings, 
whereas group was no longer a unique predictor of change in stress ratings. With respect to body dissatisfaction, group was no longer a unique predictor of change in ratings, and no other variable in the model significantly predicted change in ratings. In summary, when considering other variables that might account for response to the BIS, group remained a unique predictor of change in anxiety ratings only. These between-group differences in anxiety were consistent with pilot testing results (as shown in Appendix B).

More can be said about the association between ASI-3 scores and response to the BIS in terms of anxiety and stress ratings. Limited research has investigated the relationship between anxiety sensitivity and BN (Anestis et al., 2008). In both nonclinical and clinical (outpatients with various psychological disorders) samples, ASI-3 scores significantly predicted EDI-Bulimia scores, which links anxiety sensitivity to bingeing and purging. Effects of impulsivity, mood symptoms and anxiety symptoms were controlled, in order to isolate the link between AS and disordered eating symptoms. According to Anestis and colleagues (2008), this finding may point to a subset of individuals with an elevated fear of somatic sensations of anxiety who might eat in an effort to reduce tension and then subsequently purge. This previous study provides some support for the present finding that links AS to increased anxiety and stress following a body image related stressor.

Another question arising from the present between groups analyses is why the study groups did not differ in their ratings of fear, panic symptoms, hunger and desire to eat in response to the BIS. Given that the associated effect sizes ranged from small to large, this study may have lacked sufficient power for effects to be significantly different. Therefore, with a larger sample size, significant effects might be revealed. On the other hand, it is possible that a number of individuals in the NED group can be classified as restrained eaters, similar to those in the BN 
group; this specific construct was not measured in the present study, and it is possible that such reports were not detected during the diagnostic screening interview and self-report questionnaires of eating symptoms (due to nonendorsement by the participant). Restrained eating is generally defined as a persistent fixation on dieting, weight, and food, such that the type and amount of food eaten is restricted for the purpose of weight loss or maintenance (Ruderman, 1985). Research has demonstrated that restrained eaters react to various factors affecting eating behaviours in a manner similar to that observed in those with BN (Herman \& Policy, 1988). Notwithstanding the abovementioned explanation for the present findings (i.e., low power), change in ratings of hunger and desire to eat were not in the expected direction.

Referring more generally to all three stressor tasks, worthy of further discussion is the finding that no significant differences between groups were revealed with respect to changes in hunger and desire to eat. One marginally significant between-group finding was revealed for the TSST, as discussed above, such that individuals in the NED group became less hungry compared to individuals in the BN group, with a small effect size. All other effect sizes were trivial. Even the BN group, however, experienced decreased hunger. In fact, upon examining VAS ratings of both hunger and desire to eat, a pattern of lower scores at time 2 compared to time 1 was revealed; in other words, within both groups across all stressors, participants generally experienced a decrease in hunger levels and desire to eat following each stressor task. To emphasize, even individuals in the $\mathrm{BN}$ group reported less of a desire to eat after viewing photos of revealing clothing articles and imagining themselves wearing them.

General speculation can be made regarding these findings. First, literature on restrained eaters versus unrestrained eaters is likely relevant, in the context of the dietary restraint model. Federoff, Polivy and Herman $(1997,2003)$ demonstrated that restrained eaters had a highly 
specific response to exposure to food cues (i.e., the smell of pizza or cookies) for 10 minutes. The present study did not include food cues nor the presence of actual food. It is possible that such stimuli might have been needed to provoke an increased desire to eat or a sensation of hunger, particularly during tasks in which the participants were distracted (i.e., focusing on the task at hand). More generally, it is possible that the stressors employed in this study were not sensitive enough to provoke stress-induced eating or the desire to eat. With specific reference to the BIS, perhaps removing social factors from a task that is meant to provoke stress via effects on body image distress is not ecologically valid, and could potentially explain why the BIS did not provoke these responses as hypothesized. The BIS was developed as a stressor that was devoid of any explicit social comparison for the purpose of removing the potential influence of this confounding variable on results (in order to answer the specific research questions of this study). However, it is possible that social comparison is an important element of stress response in $\mathrm{BN}$ and necessary to provoke increased urges to binge eat. Perhaps a more implicit social comparison might have occurred during the BIS when participants were asked to focus on themselves wearing clothing articles displayed on a monitor (i.e., an imaginal exposure), even in the absence of any explicit visual cues for social comparison; however, this was not enough to provoke the expected response.

Group comparisons of physiological response to stressors. In terms of physiological (cardiovascular) response to the stressors, findings revealed no significant between-group differences on any index of stress response. This was also inconsistent with hypotheses. Given very large $p$ values (ranging from .424 to .999) and extremely small Cohen's $d$ effect sizes, it is unlikely that a larger sample would reveal significant and practically important findings. Much research evidence has accumulated regarding the physiological effects of stressors, including the 
two commonly used approaches employed in the current study (TSST and $\mathrm{CO}_{2}$ ). With respect to cardiovascular effects, based on previous research, it would be expected that the TSST would engender increased cardiovascular arousal (increased heart rate and systolic blood pressure - and to a lesser extent, diastolic blood pressure) (Allen et al., 2014); similarly, previous research would suggest the expected effects of $\mathrm{CO}_{2}$ are decreased heart rate and increased systolic blood pressure, with no effects on diastolic blood pressure (Vickers et al., 2012). While no differences were found between groups in cardiovascular stress response, results demonstrated some changes across the entire sample and also within groups; these changes were described above.

No previous study has examined cardiovascular reactions to $\mathrm{CO}_{2}$ in a $\mathrm{BN}$ sample, rendering it impossible to compare this study's findings to past work. TSST findings in the present study can be compared to those by Ginty and colleagues (2012), who found blunted HR response to the TSST in a disordered eating group compared to a control group. A similar finding was demonstrated in other studies (e.g., Koo-Loeb, Pedersen, \& Girdler, 1998). Ginty and colleagues' disordered eating sample consisted of 12 women who engaged in self-induced vomiting as a weight control behaviour; however, no formal diagnosis of $\mathrm{BN}$ was made, nor did they assess for a history of eating disorders or psychological treatment; therefore, it is difficult to accurately classify their study sample and speculate potential mechanisms of the blunted HR finding.

It is of course possible that the current study's findings are a product of some unmeasured variable (Christenfeld, Sloan, Carroll, \& Greenland, 2004). As one example, studies have demonstrated that exercise dependence has been associated with blunted cardiovascular reactivity (Heaney et al., 2011), and it is well known that exercise dependence is often comorbid with restricting and binge/purge type eating disorders (Bamber, Cockerill, \& Carroll, 2000; 
Davis et al., 1997). Exercise dependence was not assessed in the present study as a potential confounding variable, but nonetheless might have affected cardiovascular response. Perhaps the BN participants in the present study were not heavy exercisers. Historical length of the eating disorder might also have affected cardiovascular response; chronic stress, which might be present in individuals with a longer history of psychopathology, is linked to a dysregulated stress response (McEwen, 2007). Results of the present study are, however, consistent with several other studies. For example, Messerli-Burgy and colleagues (2010) found relatively high heart rate reactivity in $\mathrm{BN}$ compared to a control group of obese individuals and a binge eating disorder group. There is evidence that obesity is also associated with a blunted cardiovascular reactivity (Carroll, Phillips, \& Der, 2008) and thus individuals with BN may only appear to show elevated cardiac reactivity when compared to controls that show blunted reactivity. In the present study, the BMI of four individuals in the BN group and one individual in the NED group was in the obese range (above 30). Koo-Loeb and colleagues (2000) reported that those high in disordered eating symptoms (but not meeting a clinical diagnosis of disordered eating) displayed higher heart rate and blood pressure reactivity compared to a group low in disordered eating symptoms (Koo-Loeb, Costello, Light, \& Girdler, 2000). One other study observed no differences between those with disordered eating symptoms and controls in cardiovascular responses to stress (Cattanach \& Rodin, 1988). Taken together, these findings suggest that exercise and length of the eating disorder might be candidates worthy of further investigation to explain some of the current study's results.

More generally, results of the present study are largely inconsistent with the contention that individuals with disordered eating may have a dysregulated (suboptimal) stress response (Cattanach \& Rodin, 1988; Koo-Loeb et al., 1998; Lo Sauro, Ravaldi, Cabras, Faravelli, \& 
Ricca, 2008; Pirke et al., 1992). Of course, only cardiovascular reactivity--one of many indicators of dysregulated physiological stress response--was assessed in the current study. The present study cannot speak to other indicators (e.g., HPA axis functioning), which are important for complete understanding of a dysregulated physiological stress response, which is indeed a complex phenomenon.

\section{Social anxiety symptoms and social anxiety-related factors as predictors of stress} response in individuals with and without $\mathbf{B N}$. Irrespective of the above-mentioned betweengroup differences in stress response, it was still possible to answer this study's second major research question regarding the power of social anxiety and social anxiety related factors to predict stress reactivity. More specifically, this research question was posed for the purpose of investigating how social anxiety might differentially affect individuals with $\mathrm{BN}$ and individuals without a history of eating disorders when presented with stressful stimuli. This investigation is particularly important given two research findings: (1) high comorbidity rates between social anxiety and BN (Godart et al., 2003; Godart et al., 2000), and (2) the negative effects of interpersonal stress on BN (e.g., Hilbert, et al., 2011; Pirke et al., 1992; Vocks et al., 2007). Previous studies have demonstrated the effects of interpersonal stress in both groups (BN and clinical controls), yet no studies have specifically examined the effects of social anxiety (and related factors) on different types of stress response. A logical question that follows is whether individuals with $\mathrm{BN}$ and high levels of social anxiety experience an even greater negative response to interpersonal stress specifically, or stress in general (i.e., different types of stress). As an initial step in this investigation, scores on the SPIN and several other self-report questionnaires (measuring correlates of social anxiety) were investigated as possible predictors of response to each stressor, within each study group. 
Social anxiety symptoms. When within-participants change from time 1 to time 2 was significant within groups (as reported in Tables 8 and 10 for each subjective and physiological response), the predictive effect of social anxiety symptoms on stress response was investigated. It was hypothesized that within each group, SPIN scores would predict increased anxiety, fear, stress, and panic symptoms, as well as changes in HR, SYS-BP and DIA-BP from time 1 to time 2 of the TSST and $\mathrm{CO}_{2}$. In the BN group, hypotheses were not supported; SPIN scores alone did not predict subjective or physiological response to either stressor. On the other hand, hypotheses were partially supported within the NED group; for the TSST, SPIN scores alone predicted increases in VAS stress ratings and PSL scores. Also in response to the TSST, SPIN scores had a marginally significant predictive effect on VAS-anxiety ratings. However, when entered into a regression model with social anxiety related variables, SPIN scores were no longer significant predictors of stress ratings and panic symptom scores. Significant predictors were all associated with beta values representing small strengths of association (.2; Ferguson, 2009). Most beta values of nonsignificant predictors also represented small strengths of association; although some were below the recommended minimum effect size representing a practically significant effect.

Generally, these results do not support the contention that social anxiety significantly predicts exaggerated response to either interpersonal stress or physiological stress in those with BN. In those without a history of eating disorders, on the other hand, social anxiety symptoms did exert some effect on response to both types of stress. However, no obvious pattern emerged in these findings; future research is needed. One general possibility to consider that might have affected results is the lack of homogeneity within each group in terms of comorbid clinical 
symptoms, as well as features of the eating disorder in the BN group (e.g., severity and length of disorder history).

In response to the BIS, SPIN scores could not be tested as predictors of stress reactivity in the $\mathrm{BN}$ group, given nonsignificant within-participants change from time 1 to time 2 . In the NED group, SPIN scores were tested as predictors of panic symptoms, but findings were insignificant with an effect size below practical importance. These findings were line with hypotheses, which stated that SPIN scores were not expected to predict increases in the aforementioned outcome measures of stress response from time 1 to time 2.

Correlates of social anxiety. The predictive effect of specific correlates of social anxiety was also investigated. Fear of negative evaluation, a general indicator of social evaluative fear (Clark \& Wells, 1995; Moscovitch et al., 2009), has undergone the most extensive study in connection to BN (e.g., Striegel-Moore et al., 1993; Vander Wal \& Thomas, 2004). Social appearance anxiety is recognized as a unique domain of social anxiety focused on one's global appearance and is another construct commonly considered within the research linking BN with SAD (Koskina et al., 2011; Levinson \& Rodebaugh, 2012). Perfectionism has also been studied in the same manner; however, this construct has been measured in various ways using different scales (e.g., the FMPS and HMPS) and different combinations of subscales. In the present study, a measure of maladaptive evaluative concerns (i.e., maladaptive perfectionism) was calculated based on a factor analysis from a previous study (Bieling et al., 2004) and used along with a measure of self-oriented perfectionism (i.e., the HMPS self-oriented perfectionism subscale). Lastly, a subscale of a relatively new and validated scale, the PAPS (Yang \& Stoeber, 2012), was evaluated as an additional predictor of stress response. This particular subscale, entitled Worry about Imperfection, focuses solely on imperfection of physical appearance. 
It was hypothesized that within each study group, the aforementioned variables would predict increases in anxiety, fear, stress, and panic symptoms, as well as changes in HR, SYS-BP and DIA-BP, from time 1 to time 2 of the TSST and $\mathrm{CO}_{2}$. The opposite hypothesis (i.e., no change in the aforementioned outcome variables) was advanced for the BIS. Again, withinparticipants change from time 1 to time 2 had to be significant in order to investigate possible predictors of this change. With this in mind, only social appearance anxiety on the SAAS was revealed as a significant predictor of panic symptoms, alongside SPIN scores, in response to $\mathrm{CO}_{2}$. No other significant predictors were revealed and effect sizes ranged from not practically significant to a moderate effect with no obvious pattern, and therefore, for the most part, findings were inconsistent with hypotheses.

Overall, these results do not support the contention that social anxiety related factors significantly predict subjective or cardiovascular response to any of the three stressor types in the present study. Furthermore, it was difficult to speculate reasons for the abovementioned findings, as no response patterns were evident. Baseline clinical characteristics of the study sample were examined to consider whether means and standard deviations of scores on self-report measures were comparable to those previously published within similar samples. Overall, participants in the present sample did not report unusually low scores that might have been able to explain the current findings. For example, total scores on the BFNE in the present BN sample were comparable to those in a SAD outpatient sample $(M=46.91, S D=9.27)$ and those in the present NED sample were slightly higher than nonanxious controls $(M=26.81, S D=4.78$; Weeks et al., 2005). In terms of self-oriented perfectionism, scores in the present BN sample were slightly higher than a sample of female psychiatric outpatients $(M=72.48, S D=17.96)$ and the NED 
group was slightly lower than a female community sample $(M=69.87, S D=14.83$; Hewitt et al., 1991).

\section{Associations among psychological symptoms (social anxiety, depression, trait}

anxiety) that commonly co-occur with $\mathrm{BN}$ and response to stressors. Since the overall goal of this research was to better understand factors that affect stress response in $\mathrm{BN}$, this study sought to answer several additional questions specifically within the BN group. First, BN is not only highly comorbid with social anxiety but also with depression and trait levels of anxiety; therefore, it is prudent to consider the effects of these other common comorbid conditions on stress response in this population. Findings demonstrated that neither variable (DASS-21-D scores or STICSA-Trait scores) was found to correlate significantly with any of the psychological or physiological stress response indicators in the present study. This appears inconsistent with the abovementioned findings from regression analyses, although when DASS-21-D scores were placed in a regression model their predictive effect was considered alongside other possible predictors, rather than alone.

\section{Associations among stress reactivity (anxiety, fear, stress, panic symptoms) and} body image/eating related responses (desire to eat, hunger, body dissatisfaction). While it is important to understand the response to stress in $\mathrm{BN}$, it is also necessary to determine whether the experience of stress is related to an increase in subsequent binge eating behaviour. Therefore, this study examined whether subjective stress reactivity (VAS ratings of anxiety, fear and stress, and PSL scores) was associated with eating/body image related responses (VAS ratings of hunger, desire to eat, and body dissatisfaction). In response to $\mathrm{CO}_{2}$, greater levels of anxiety were correlated with lower levels of body dissatisfaction. Additionally, in response to the TSST, greater levels of fear were correlated with greater levels of body dissatisfaction. No other 
significant associations were revealed. When a Bonferroni correction was applied to these data to decrease the likelihood of a type I error given multiple comparisons, these associations were no longer significant. Contrary to hypotheses, these results do not demonstrate a connection between exaggerated stress response and disordered eating cognitions/symptoms. As discussed above, perhaps the lack of a presence of food cues or actual food as part of the stressor might partially explain these findings.

Differential stressor effects in individuals with BN. The final goal of the present study was to explore within-participants effects of the three stressors in the BN group, to determine the extent to which change in each subjective response indicator was similar across the three stressor tasks. No a priori hypotheses were advanced due to a dearth of existing research on which to base predictions. Findings revealed no significant differences, indicating that participants in the BN group experienced similar reactions (i.e., similar degrees of change in each response indicator) across all three stressor tasks. While no conclusions should be drawn from this finding without replication, these results are in line with Selye's (1950) seminal perspective on the general stress response, such that all stressors of similar intensity engender a nonspecific response that is equivalent across stressors. That noted, more recent research in nonclinical populations has revealed some variations in stress response depending on type of stressor (Dickerson \& Kemeny, 2004; Maruyama et al., 2012; Skoluda et al., 2015), which is inconsistent with the current findings in BN. Further research on stress response to different stressors in BN will elucidate matters.

\section{Implications of the Present Research}

Considering the various distinct findings of this study, some general implications can be made. Research regarding evidence-based treatment practices for BN has flourished over the past 
decade, most notably Cognitive Behavioural Therapy for eating disorders (Fairburn, 2008). However, treatment outcome studies indicate a continued need to enhance BN treatment efficacy given high rates of partial response, high dropout rates and considerable relapse (Fichter \& Quadflieg, 2007; Herzog et al., 1999; Mitchell, Hoberman, Peterson, Mussell, \& Pyle, 1996; Shapiro et al., 2007; Stice, Marti, Shaw, \& Jaconis, 2009). In addition to furthering the study of stress reactivity in $\mathrm{BN}$ compared to NED, a second major objective of the present study was to better understanding the relationship between $\mathrm{BN}$ and social anxiety symptoms - and more specifically, how this relationship might affect stress response. If researchers and clinicians can better understand what types of stress might provoke an exaggerated response in those with $\mathrm{BN}$, and what factors might influence this response, then perhaps clinical implications can follow that might subsequently improve the treatment success rate. With that said, the mechanisms that maintain BN symptoms is an important topic of research, and a recent trajectory of studies has investigated social anxiety as one such potential mechanism. This research is distinct from that which has previously focused on interpersonal stress as a predictor of BN symptoms.

Interpersonal stress can indeed affect individuals with BN both with and without high levels of comorbid social anxiety symptoms. Given that the core psychopathology of BN involves an overevaluation of weight and shape (which often involves social comparison), it seems obvious that interpersonal stress would provoke a negative reaction, which might entail binge eating as an affect regulation strategy. However, it is possible that those with $\mathrm{BN}$ and high levels of social anxiety symptoms might react to interpersonal stress in a unique way that might have implications for treatment. It can be argued that any comorbid psychopathology would put one at greater risk for exaggerated stress response and unsuccessful treatment, which is why 
other common comorbid symptoms were considered in the present study. Present study findings did not support this contention.

More generally, in all individuals with BN regardless of comorbidity, stress is shown to provoke binge eating, which serves as a coping mechanism and also an emotion regulation strategy for stress and related negative affect (Whiteside et al., 2007). Along these lines, several research teams have reported that at least $50 \%$ of binges are affect driven, rather than due to hunger (Greeno, Wing, \& Shiffman, 2000; Fairburn, 1997), and that binge eating is associated with a decrease in negative affect (Deaver, Miltenberger, Smyth, \& Crosby, 2003). This idea is supported by affect models of binge eating, which implicate negative affect as the most common instigator of binge-eating episodes (Polivy \& Herman, 1993), in contrast to dietary restraint models.

The cognitive-behavioural theory of eating disorders, on the other hand, is more consistent with dietary restraint models. This theory emphasizes the powerful perpetuating cycle of food restriction, binge eating, and compensatory behaviours. According to the theory, binge eating is largely a result of attempts to adhere to extreme dietary rules; this behaviour is not an important part of the psychopathology itself. The core psychopathology of $\mathrm{BN}$ is an overevaluation of weight and shape and their control. During laboratory based stressor tasks, if response is not affect driven, then an urge to binge eat would not be expected unless food cues or actual foods are presented to participants that cue hunger. This makes intuitive sense in that if an individual who has been restricting food intake is presented with a stimulus that cues hunger, a desire to eat would naturally follow. Although the current study did not measure binge eating as a behavioral response, it did demonstrate that stress does not provoke increased hunger and desire to eat in individuals with $\mathrm{BN}$; this finding does not contradict the cognitive behavioural 
model of eating disorders. Rather, it implies that affect regulation did not represent the function of bingeing in participants of the present study. Distress related to the core psychopathology of BN (overevaluation of weight and shape) was likely present, and accordingly, the immediate response was an urge to restrict (rather than an urge to binge).

Increased perceptions of stress can precede binges, as demonstrated in a number of studies (e.g., Cattanach \& Rodin, 1988). The present study findings do not contradict these previous findings. Rather, they simply do not implicate bingeing as being proximal to the stressful event. Perhaps stress initially increases the overevaluation of weight and shape (as indicated by decreased hunger and desire to eat), and then based on various mediating factors (e.g., the nature of the food restriction) the bingeing might occur later on, as a more distal response. Continued research using laboratory stressor tasks as well as ecological momentary assessment of stressful events can more carefully elucidate proximal versus distal factors in relation to binge episodes. This type of research has been furthered in recent years (e.g., Goldschmidt et al., 2014; Smyth et al., 2007; Smyth et al., 2009).

\section{Strengths and Limitations of This Study}

This study has a number of strengths. This is the first known study involving an experimental manipulation to investigate relationships among stress response, social anxiety and social anxiety correlates. A number of previous studies have looked at how interpersonal stressors affect those with BN, but taking into account actual symptoms of social anxiety is novel. Several factors known to affect stress response also were taken into account. These include perceived stress, actual life stress (stressful events), anxiety sensitivity, depressed mood, trait anxiety, and state anxiety. It should be noted, however, that the measure of state anxiety in the present study was not a true baseline measure of state anxiety as this questionnaire was 
completed midway through the testing session. This represents a limitation of the present study's methodology. Nonetheless, consideration of each of the above-mentioned factors served to enhance confidence in the findings of significant between group differences in stress response. Of course, not all possible psychological risk factors for stress response can be considered. Some that were not considered but were mentioned above to have potentially affected stress response in the present study include historical length of the eating disorder and exercise dependence.

This is also the first study to compare response to multiple laboratory stressors in a BN sample. More specifically, this study's methodology allowed for comparisons of response (both emotional and physiological) to three distinct stressor tasks in those with BN. A wide variety of experimental manipulations of stress response have been used in laboratory research; the present study supports the historical contention that all stressors are roughly equivalent (at least in a BN population) in light of the trivial effect sizes associated with comparisons among the three stressors. Without overinterpreting null findings, the best conclusion is that the hypothesis of differences across stressor response in BN was not supported in the current study and a possible reason for this finding is that other research with contradictory findings (e.g., Maruyama et al., 2012; Skoluda et al., 2015) did not use a BN sample. The present study also provided initial evidence supporting the use of $\mathrm{CO}_{2}$ as a laboratory stress test in a psychiatric population, namely, BN. Of course, further studies are needed before concluding that the $\mathrm{CO}_{2}$ test is a valid stress provocation in $\mathrm{BN}$.

Limitations of the current study are also noteworthy. As reported above, the proportions within each group of individuals with diagnosed social anxiety were small, and analyses relied on social anxiety symptoms from the SPIN, a self-report severity measure, to address study hypotheses. Perhaps if the BN group consisted of more individuals with a diagnosis of SAD, 
rather than merely high levels of social anxiety symptoms, findings might have demonstrated exaggerated response to an interpersonal stressor in comparison to other stressors. In future studies, it would be useful to recruit a group of comorbid SAD and BN compared to BN alone to determine whether the addition of an SAD diagnosis makes a difference in response to an interpersonal stressor, or whether interpersonal stress is simply inherent in BN regardless of the presence of a distinct SAD diagnosis. Either with a sample of this type, or one with a wider range of severity of social anxiety symptoms, analyses could adequately test whether social anxiety related traits serve as moderators of the stress-bulimic behaviour association (see Figure 1). More generally, a larger sample size would have generated more confidence in study findings; some analyses were low in statistical power. Wwith VAS-anxiety as the outcome variable, for example, the sample sizes required for a desired power of .80 with an alpha of .05 are as follows: $N=101$ for the TSST, $N=229$ for $\mathrm{CO}_{2}$, and $N=85$ for the BIS. In addition, the sample was exclusively female, which raises the issue of generalization. However, disordered eating is much more prevalent and better characterized in females than males (Woodside et al., 2001). Another characteristic of the sample that may limit generalizability is the number of individuals that were excluded from study participation due to medical requirements of the $\mathrm{CO}_{2}$ test.

Of course, as previously mentioned, a number of factors could have affected response to the stressors, and all possible factors cannot be considered. However, one factor that is often considered in stress research that was not in the present study was menstrual cycle (and more generally, pre or postmenopausal status). It is common in studies of stress response to determine participants' menstrual cycle phase at the time of study completion, as this variable can also affect stress response (Monteleone et al., 2012). An additional limitation was that participants were not asked if they believed there was a social component to the BIS. Although there was no 
explicit social component to this stressor, it is not known whether implied social forces were potentially affecting response to the BIS. This information would be useful when interpreting study findings. Another potential limitation concerns the use of different measurement tools for cardiovascular data across studies of stress response, (and, accordingly, the acquisition of continuous versus discrete data), which might partially explain inconsistent results across studies.

\section{Conclusion}

The present study did not find strong evidence for significant between-group differences in stress reactivity; however, effect size estimates revealed that individuals with BN tend to experience enhanced stress reactivity compared to an eating disorder control group on some measures of response. Additionally, in individuals with BN, response was similar to all three types of stress tested that were provoked. Moreover, neither SA symptoms nor associated factors predicted stress reactivity in either group. Perhaps the common factors of BN and SAD affect the eating disorder course via an alternative method that was not tested in the current study, which can be elucidated through future research. Considering the findings, strengths, and limitations of this study, experimental research should also continue to investigate stress response in $\mathrm{BN}$, in an attempt to elucidate factors that predict enhanced response and subsequent BN symptomatology. 
Figure 1.

Moderational model of the relationship between bulimic behaviours and stress response

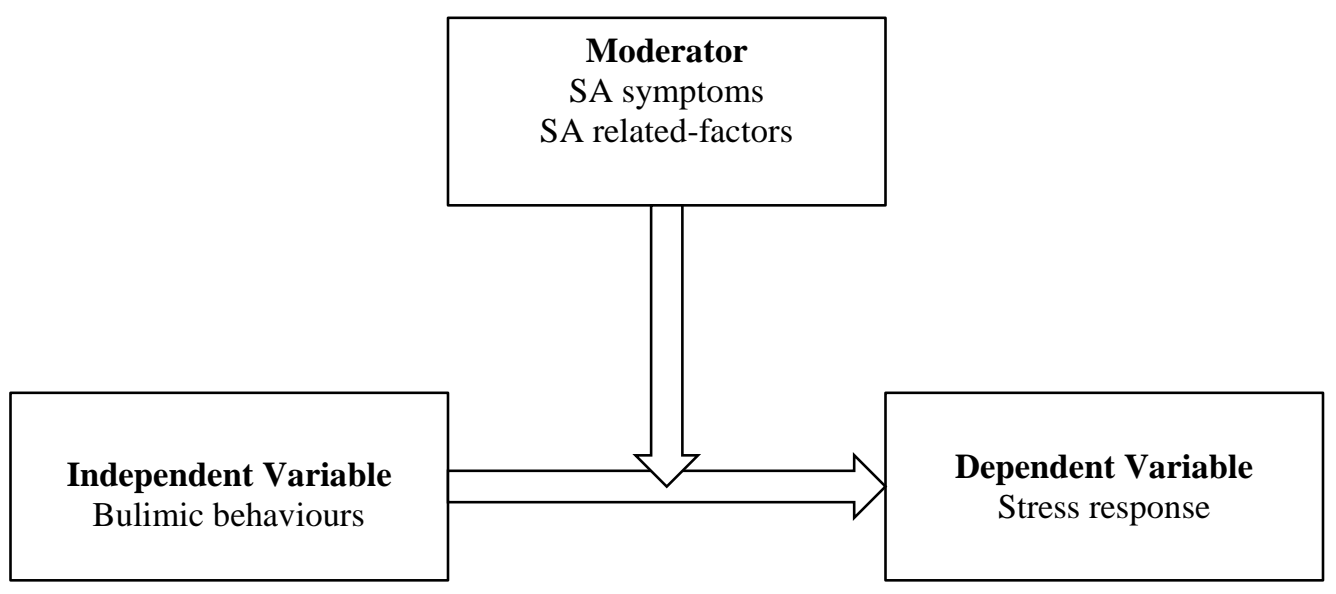

Note. $\mathrm{SA}=$ social anxiety 


\section{Appendix A}

Pilot Testing the Effects of a Newly Developed Body-Image Stressor

The BIS was developed specifically for use in this dissertation. For this reason, pilot testing was first conducted to examine the validity of its use as a laboratory stressor. The following is a summary of the methodology used for this pilot testing and associated results, which were part of a larger, ongoing study.

A total of 39 females participated in this pilot testing. All participants were recruited from Ryerson University’s Introductory Psychology Research Participant Pool through Sona, an online management system. They received partial course credit for participation. Any female who expressed an interest in this study was eligible to participate; allocation to specific study groups was determined after data collection. A practical limitation of using the university's research participant pool was the inability to thoroughly assess eating disorder diagnostic criteria of potential participants before they arrived at the laboratory to complete the study. However, since research has demonstrated a positive association between negative body image and disordered eating more generally, it was determined that the clinical group within this pilot testing would not be limited to those with a diagnosis of $\mathrm{BN}$.

For those who presented to the Psychophysiology Laboratory to complete the study, the following procedure was undertaken. Participants first responded to a series of self-report measures that were presented on a computer screen via Qualtrics, an internet-based survey company. The following subset of measures was used for the analyses reported below: the Body Shape Questionnaire (BSQ; Cooper et al., 1987), the Eating Attitudes Test - 26 item version (EAT-26; Garner \& Garfinkel, 1979; Garner et al., 1982), and the Eating Disorders Inventory 
(EDI; Garner, Olmstead, \& Polivy). Following questionnaire completion, participants underwent the stressor task. This task is described in detail within the methods section of this dissertation.

Response to the BIS was evaluated by the same outcome measures as those within the main study of this dissertation: (1) visual analogue scales (VAS), each on a continuum ranging from 0 (none at all) to 100 (the worst imaginable), to measure the degree of subjective intensity of the following sensations: anxiety, fear, stress, body dissatisfaction, hunger, desire to eat; (2) the Panic Symptoms List (PSL; Schruers et al., 2000), a self-report questionnaire that assesses each of the 13 symptoms of physical and cognitive arousal associated with spontaneous panic attacks. Participants completed these measures at two time points: immediately before undergoing the stressor task (time 1, pre stressor), and immediately upon its completion (time 2, post stressor). The post-stressor measurement represented peak response to the stressor task.

The following exploratory research questions were advanced to determine whether the BIS had a subjective effect on individuals with disordered eating, and moreover, whether this effect was significantly greater in those with disordered eating compared to those without disordered eating. (1) In each group, was there a change in VAS ratings of anxiety, fear, and stress, as well as PSL scores from time 1 to time 2? (2) Compared to the healthy eaters group, did the disordered eaters group demonstrate greater increases in VAS ratings of anxiety, fear, and stress, as well as PSL scores from time 1 to time 2? (3) Compared to the healthy eaters group, did the disordered eaters group demonstrate greater increases in VAS ratings of body dissatisfaction, hunger, and desire to eat?

Data from a total of 39 females were collected. Following the completion of data collection, participants were divided into one of two groups based on their EAT-26 total score. Those who scored $\geq 20$ were allocated to a disordered eaters group $(n=10)$, and those who 
scored $<20$ were allocated to a healthy eaters group $(n=29)$. Scores on the BSQ were then examined. Scores greater than 80 on the BSQ are indicative of higher than normal levels of body shape concerns; therefore, participants in the healthy eaters group with BSQ scores greater than 80 were not considered for analyses. Based on this criterion, 15 individuals were removed from the non-disordered eaters group. One additional individual was removed from the disordered eaters group because she had a particularly low BSQ score, which constituted an outlier in the data (greater than 3 standard deviations away from the mean).

Data from a total of 23 females were used in analyses ( 9 disordered eaters, 14 healthy eaters). Participants ranged in age from 18 to 60 years old $(M=23.43, S D=9.06)$. The majority of participants reported their ethnicity as White (34\%), followed by South Asian (30.4\%), Arab or West Asian (13\%), East Asian (8.7\%), and Black (8.7\%). One additional participant did not wish to disclose her ethnicity (4.3\%). Table 1 depicts demographic and disordered eating baseline characteristics of participants, separated by study group.

Two-tailed independent samples $t$-tests revealed that the two study groups did not differ in age, $t(21)=1.40, p=.175, r_{\mathrm{pb}}=.29$, or ethnicity, $\chi^{2}(5)=5.13, p=.400, \phi_{c}=.472$. In terms of disordered eating, scores on the EAT-26 were significantly greater in the disordered eaters group compared to the healthy eaters group, $t(19.71)=9.57, p<.001, r_{\mathrm{pb}}=.89$ (degrees of freedom were corrected for unequal variances identified by Levene's test). Scores on the BSQ were also significantly greater in the disordered eaters group compared to the healthy eaters group, $t(21)=$ $-10.66, p<.001, r_{\mathrm{pb}}=.92$. Moreover, scores on the EDI-Bulimia Subscale in the disordered eaters group were significantly greater than those in the healthy eaters group, $t(21)=4.13, p<$ $.001, r_{\mathrm{pb}}=.67$. 
Table 2 displays the means and standard deviations of VAS and PSL scores at time 1 and time 2, separated by group. Within each group, paired-samples $t$-tests were first performed to examine the main effects of time on the following four subjective stress response indicators: (1) VAS ratings of anxiety, fear and stress; (2) panic symptom scores on the PSL. Within-subjects effects of time were not revealed for either group (as seen in Table 3). Participants did not report statistically significant changes in VAS ratings of anxiety, fear, stress and PSL scores from time 1 to time 2 . However, the Cohen's $d$ effect sizes (Cohen, 1988, 1992) for change in VAS ratings of anxiety (depicted in Table 2) within each group were practically significant effects (Ferguson, 2009). Interestingly, the direction of change in the disordered eaters group was such that anxiety increased from time 1 to time 2, whereas in the healthy eaters group anxiety decreased from time 1 to time 2 .

Residualized change scores (Steketee \& Chambless, 1992) were then calculated from time 1 to time 2 for each measure of response, in order to control for initial differences and measurement error inherent in the use of repeated measures on the same instrument. Table 4 displays the results of independent samples $t$-tests for each of the seven response indicators, including $t$ statistics, associated $p$ values and Cohen's $d$ effect size estimates (Cohen, 1988, 1992). Compared to the healthy eaters group, the disordered eaters group reported significantly greater increases in VAS ratings of anxiety from time 1 to time 2 with a moderate effect size. No other significant between-group differences were observed. 
Table 1

Demographics and Baseline Clinical Characteristics of the Study Sample

\begin{tabular}{lcc}
\hline Variable & $\begin{array}{c}\text { Disordered Eaters } \\
(n=9)\end{array}$ & $\begin{array}{c}\text { Healthy Eaters } \\
(n=14)\end{array}$ \\
\hline Age in years - $M(S D)$ & $26.67(3.17)$ & $21.36(4.48)$ \\
Ethnicity - Frequency (\%) & $3(33.3)$ & $5(35.7)$ \\
$\quad$ White & $3(33.3)$ & $4(28.6)$ \\
$\quad$ South Asian & $2(22.2)$ & $1(7.14)$ \\
$\quad$ Arab or West Asian & $0(0)$ & $2(14.3)$ \\
$\quad$ East Asian & $0(0)$ & $2(14.3)$ \\
Black & $1(0)$ & $0(0)$ \\
Other & $30.44(8.23)$ & $6.36(4.94)$ \\
EAT - 26* & $133.88(21.36)$ & $58.21(12.86)$ \\
BSQ* & $7.22(5.83)$ & $0.57(1.45)$ \\
EDI - Bulimia Subscale* & & \\
\hline $\begin{array}{l}\text { Note. EAT-26 = Eating Attitudes Test - 26 item version; BSQ = Body Shape Questionnaire; EDI } \\
=\text { Eating Disorder Inventory. } \\
* \text { < .001 }\end{array}$ & & \\
& &
\end{tabular}


Table 2

Means and Standard Deviations of Subjective Stress Response Scores for the BIS, Separated by Study Group (Raw Scores)

\begin{tabular}{lcccc}
\cline { 2 - 4 } & \multicolumn{2}{c}{ Disordered Eaters $(n=9)$} & \multicolumn{2}{c}{ Healthy Eaters $(n=14)$} \\
\hline Outcome Variable & Pre & Post & Pre & Post \\
\hline VAS-anxiety & $42.89(28.13)$ & $49.22(32.30)$ & $13.00(18.15)$ & $8.93(15.17)$ \\
VAS-fear & $28.89(30.49)$ & $29.11(32.27)$ & $2.29(4.73)$ & $1.50(2.93)$ \\
VAS-stress & $76.00(18.06)$ & $75.22(19.14)$ & $18.43(24.76)$ & $18.64(27.35)$ \\
VAS-hunger & $26.33(29.82)$ & $18.44(26.54)$ & $37.79(34.20)$ & $36.21(34.62)$ \\
VAS-desire to eat & $30.89(34.91)$ & $18.00(25.85)$ & $37.14(32.71)$ & $31.21(35.88)$ \\
VAS-body dissatisfaction & $72.78(26.42)$ & $76.78(25.17)$ & $6.29(16.33)$ & $17.86(22.22)$ \\
PSL & $6.00(5.45)$ & $6.78(4.37)$ & $2.79(3.75)$ & $2.86(3.21)$ \\
\hline
\end{tabular}

Note. BIS = body image stressor; VAS = visual analogue scale; PSL = Panic Symptoms List . 
Table 3

Results of Paired Samples T-Tests of Time 1 and Time 2 Scores on Subjective Measures of Stress Response, Separated by Group

\begin{tabular}{lcccccc} 
& \multicolumn{3}{c}{ Disordered Eaters $(n=9)$} & \multicolumn{3}{c}{ Healthy Eaters $(n=14)$} \\
\hline & $t(8)$ & $p$-value & $d$ & $t(8)$ & $p$-value & $d$ \\
\hline VAS-anxiety & 1.30 & .229 & .451 & -1.64 & .126 & -.460 \\
VAS-fear & 0.07 & .950 & .022 & -0.85 & .409 & -.262 \\
VAS-stress & -0.22 & .830 & -.075 & 0.07 & .947 & .018 \\
PSL & 0.84 & .425 & .285 & 0.20 & .844 & .058 \\
\hline
\end{tabular}

Note. $\mathrm{VAS}=$ visual analogue scale; PSL = Panic Symptoms List. 
Table 4

Independent Samples T-Tests Comparing Subjective Responses to Each Stressor Task in the Disordered Eaters Group Compared to the Healthy Eaters Group

\begin{tabular}{lcccc}
\cline { 2 - 4 } & $d f$ & $t$ & $p$-value & $d$ \\
\hline VAS-anxiety & 21 & 2.50 & $.021^{*}$ & 1.068 \\
VAS-fear & 9.19 & 0.31 & .801 & .132 \\
VAS-stress & 21 & 0.80 & .436 & .342 \\
PSL & 9.99 & 1.61 & .200 & .688 \\
VAS-hunger & 10.09 & -1.81 & .101 & -.773 \\
VAS-desire to eat & 21 & -1.00 & .328 & -.427 \\
VAS-body dissatisfaction & 21 & 0.15 & .885 & .064 \\
\hline
\end{tabular}

Note. VAS = visual analogue scale; PSL = Panic Symptoms List. 


\section{Appendix B}

\section{Stress-Induction Studies Comparing VAS Ratings Pre and Post-CO $\mathrm{CO}_{2}$}

\begin{tabular}{|c|c|c|c|c|}
\hline Publication & $\begin{array}{l}\text { Argyropoulos et } \\
\text { al. (2002) }\end{array}$ & $\begin{array}{l}\text { Wetherell et al. } \\
(2006)^{*}\end{array}$ & $\begin{array}{l}\text { Hood et al. } \\
\text { (2006) }\end{array}$ & $\begin{array}{l}\text { Shufflebotham } \\
\text { et al. (2009) }\end{array}$ \\
\hline $\begin{array}{l}\text { Participant } \\
\text { sample }\end{array}$ & $\begin{array}{l}10 \text { healthy } \\
\text { volunteers }\end{array}$ & $\begin{array}{l}24 \text { healthy } \\
\text { volunteers }\end{array}$ & $\begin{array}{l}14 \text { healthy } \\
\text { volunteers } \\
\text { (non- } \\
\text { tryptophan } \\
\text { depletion } \\
\text { condition) }\end{array}$ & $\begin{array}{l}10 \text { healthy } \\
\text { controls }\end{array}$ \\
\hline $\begin{array}{l}\text { Placebo } \\
\text { controlled? }\end{array}$ & Yes & No & Yes & No \\
\hline Anxious & -- & $18 / 53$ & $3.0 / 28.6$ & $12.3 / 38.0$ \\
\hline Fearful & $17.1 / 40.7$ & $9 / 31$ & -- & -- \\
\hline Breathless & -- & $2 / 58$ & -- & -- \\
\hline Relaxed & $40.7 / 20.7$ & $55 / 32$ & -- & -- \\
\hline Happy & $47.9 / 27.5$ & $62 / 44$ & -- & -- \\
\hline $\begin{array}{l}\text { Feel like } \\
\text { leaving the room }\end{array}$ & $5.7 / 13.6$ & -- & -- & -- \\
\hline Feel paralyzed & $4.62 / 16.2$ & -- & -- & -- \\
\hline Nervous & -- & -- & -- & $16.0 / 28.5$ \\
\hline Tense & -- & -- & -- & $16.0 / 30.0$ \\
\hline Alert & -- & $61 / 53$ & -- & -- \\
\hline Nauseated & -- & $6 / 25$ & -- & -- \\
\hline Palpitations & -- & -- & $0.0 / 23.6$ & -- \\
\hline $\begin{array}{l}\text { Something bad is } \\
\text { going to happen }\end{array}$ & -- & -- & $0.4 / 9.3$ & -- \\
\hline
\end{tabular}

Note. VAS = visual analogue scale.

Note. VAS were rated on a $100-\mathrm{mm}$ scale.

Note. $\mathrm{CO}_{2}$ studies of stress response not included in this table were those

*Reported values are estimates based on graphical data presented within published papers. 


\section{Appendix C}

\section{Effect Sizes of Reactivity Comparisons Amongst Bulimia Nervosa, Panic Disorder, and Healthy Control Groups, Using VAS-A Change as The Outcome Variable}

\begin{tabular}{|c|c|c|c|c|}
\hline Publication & $\mathrm{M}(\mathrm{SD})$ of VAS change & Group comparisons & Cohen's $d$ & Sample size \\
\hline Woznica et al. (2015) & $\begin{array}{l}\text { HC: }-10.32(25.23) \\
\text { BN: } 11.04(14.61) \\
\text { PD: } 7.18(28.24)\end{array}$ & $\begin{array}{l}\text { HC vs. } \mathrm{BN}^{* *} \\
\mathrm{HC} \text { vs. } \mathrm{PD}^{* *} \\
\text { PD vs. } \mathrm{BN}^{-}\end{array}$ & $\begin{array}{l}d=0.73 \\
d=0.62 \\
d=0.12\end{array}$ & $\begin{array}{l}30 \mathrm{HC} \\
14 \mathrm{BN} \\
15 \mathrm{PD}\end{array}$ \\
\hline Schutters et al. (2012) & $\begin{array}{l}\text { HC: } 13.7(22.4) \\
\text { SAD: } 22.6(36.1) \\
\text { PD: } 48.4(27.4)\end{array}$ & $\begin{array}{l}\mathrm{HC} \text { vs. } \mathrm{SAD} \\
\mathrm{PD} \text { vs. } \mathrm{SAD} * * *\end{array}$ & $\begin{array}{l}d=0.30 \\
d=0.81\end{array}$ & $\begin{array}{l}48 \text { total } \\
\text { (16/group) }\end{array}$ \\
\hline Caldirola et al. (1997) & $\begin{array}{l}\text { HC: } 7.8(32.8) \\
\text { SAD: } 61.3(33.3) \\
\text { PD: } 54.6(37.4)\end{array}$ & $\begin{array}{l}\mathrm{HC} \text { vs. } \mathrm{SAD} \mathrm{AD}^{* * *} \\
\mathrm{PD} \text { vs. } \mathrm{SAD} * * *\end{array}$ & $\begin{array}{l}d=1.62 \\
d=0.19\end{array}$ & $\begin{array}{l}48 \text { total } \\
\text { (16/group) }\end{array}$ \\
\hline Papp et al. (1993) & $\begin{array}{l}\text { HC: } 1.36(2.28) \\
\text { SAD: } 2.47(2.61) \\
\text { PD: } 3.33(2.66)\end{array}$ & $\begin{array}{l}\mathrm{HC} \text { vs. } \mathrm{SAD}^{* * *} \\
\mathrm{PD} \text { vs. } \mathrm{SAD}^{*}\end{array}$ & $\begin{array}{l}d=0.45 \\
d=0.33\end{array}$ & $\begin{array}{l}23 \mathrm{HC} \\
20 \mathrm{SAD} \\
18 \mathrm{PD}\end{array}$ \\
\hline
\end{tabular}

Note. $\mathrm{HC}=$ healthy controls; $\mathrm{BN}=$ bulimia nervosa $\mathrm{PD}=$ panic disorder; $\mathrm{SAD}=$ social anxiety disorder; $\mathrm{M}=$ mean; $\mathrm{SD}=$ standard deviation

Note. Papp et al. (1993) used a 10-point VAS scale; all other studies used a 100-point scale.

Note. In Woznica et al. (2015), calculations by Perna and colleagues (e.g., Perna et al., 2004) were applied to VASA raw change scores prior to analyses.

Note. These group comparisons were made via posthoc testing after significant ANOVAs were found within each study; however, it was not possible to calculate effect sizes from these posthoc tests based on the available information. Therefore, the effect sizes $(d)$ reported in this table are those of independent t-tests using the means and standard deviations provided.

$* p<.001 ; * * p<.01 ; * * * p<.05$ 


\section{Appendix D}

\section{Summary of Medical History Exclusion Criteria}

Medical exclusion criteria for all participants will be consistent with $\mathrm{CO}_{2}$ studies conducted in other laboratories (e.g., Schmidt et al., 2008). They are as follows: (a) current pregnancy; (b) personal medical history of brain tumor, cerebral aneurysm, cerebral hemorrhage, stroke, transient ischemic attack, heart attack, heart disease, coronary artery disease, congestive heart failure, mitral valve prolapse, diabetes, history of fainting (vasovagal syncope or otherwise unexplained fainting episodes) renal disease, heart murmur, cardiac arrhythmia, respiratory disease, lung disease, basilar artery migraine, asthma, epilepsy, hemiplegic migraine, seizures, liver disease, kidney disease, ophthalmoplegic migraine, hypertension, or cerebrovascular accident; (c) family history (first degree relatives) of cerebral aneurysm, cerebral hemorrhage, or hemiplegic migraine; (d) endorsement of any of the three headache symptom questions that screen for complicated migraine (refer to MHQ, question 7); (e) use of psychotropic medications except for benzodiazepines occasionally (i.e., less than twice a week and not within five half lives of the challenge); and (f) use of a medication that can significantly affect heart rate (e.g., beta-blockers, calcium channel blockers, tricyclic antidepressants).

These exclusion criteria are necessary for three reasons: to make sure that no participants have a personal history of any medical conditions or a family history of highly heritable medical conditions that might theoretically pose a risk in the $\mathrm{CO}_{2}$ challenge; [criteria (b) and (d)]; to make sure that no participants are taking medications known to minimize their reaction to the $\mathrm{CO}_{2}$ challenge [criterion (e)]; and to make sure that no participants are taking medications whose effect on heart rate might theoretically be a risk during the $\mathrm{CO}_{2}$ challenge [criterion (f)]. 


\section{References}

Allen, K., Blascovich, J., \& Mendes, W. B. (2002). Cardiovascular reactivity and the presence of pets, friends, and spouses: The truth about cats and dogs. Psychosomatic Medicine, 64, 727-739. doi:10.1097/01.PSY.0000024236.11538.41

Allen, A. P., Kennedy, P. J., Cryan, J. F., Dinan, T. G., \& Clarke, G. (2014). Biological and psychological markers of stress in humans: Focus on the Trier Social Stress Test. Neuroscience and Biobehavioral Reviews, 38, 94-124. doi:10.1016/j.neubiorev.2013.11.005

American Psychiatric Association (2013). Diagnostic and statistical manual of mental disorders (5th edition). Arlington, VA: American Psychiatric Association.

Anderson, E. R., \& Hope, D. A. (2009). The relationship among social phobia, objective and perceived physiological reactivity, and anxiety sensitivity in an adolescent population. Journal of Anxiety Disorders, 23, 18-26. doi:10.1016/j.janxdis.2008.03.011

Anestis, M. D., Holm-Denoma, J. M., Gordon, K. H., Schmidt, N. B., \& Joiner, T. E. (2008). The role of anxiety sensitivity in eating pathology. Cognitive Therapy and Research, 32, 370-385. doi:10.1007/s10608-006-9085-y

Antony, M. M., Bieling, P. J., Cox, B. J., Enns, M. W., \& Swinson. R. P. (1998). Psychometric properties of the 42-item and 21-item versions of the Depression Anxiety Stress Scales in clinical groups and a community sample. Psychological Assessment, 10, 176-181. doi:10.1037/1040-3590.10.2.176

Antony, M. M., Coons, M. J., McCabe, R. E., Ashbaugh, A., \& Swinson, R. P. (2006). Psychometric properties of the Social Phobia Inventory: Further evaluation. Behavour Research and Therapy, 44, 1177-1185. doi:10.1016/j.brat.2005.08.013 
Antony, M. M., Purdon, C. L., Huta, V., \& Swinson, R. P. (1998). Dimensions of perfectionism across the anxiety disorders. Behaviour Research and Therapy, 36, 1143-1154. doi:10.1016/S0005-7967(98)00083-7

Arcelus, J., Haslam, M., Farrow, C., \& Meyer, C. (2013). The role of interpersonal functioning in the maintenance of eating psychopathology: A systematic review and testable model. Clinical Psychology Review, 33, 156-167. doi: 10.1016/j.cpr.2012.10.009

Arcelus, J., Mitchell, A. J., Wales, J., \& Nielsen, S. (2011). Mortality rates in patients with anorexia nervosa and other eating disorders. A meta-analysis of 36 studies. Archives of General Psychiatry, 68, 724-731. doi:10.1001/archgenpsychiatry.2011.74

Argyropoulos, S. V., Bailey, J. E., Hood, S. D., Kendrick, A. H., Rich, A. S., Laszlo, G., . . . Nutt, D. J. (2002). Inhalation of $35 \% \mathrm{CO}_{2}$ results in activation of the HPA axis in healthy volunteers. Psychoneuroendocrinology, 27, 715-729. doi:http://dx.doi.org/10.1016/S0306-4530(01)00075-0

Ashbaugh, A., Antony, M. M., Liss, A., Summerfeldt, L. J., McCabe, R. E., \& Swinson, R. P. (2007). Changes in perfectionism following cognitive-behavioral treatment for social phobia. Depression and Anxiety, 24, 169-177. doi:10.1002/da.20219

Bamber, D., Cockerill, I. M., \& Carroll, D. (2000). The pathological status of exercise dependence. British Journal of Sports Medicine, 34, 125-132.

Bardone-Cone, A. M., Wonderlich, S. A., Frost, R. O., Bulik, C. M., Mitchell, J. E., Uppala, S., \& Simonich, H. (2007). Perfectionism and eating disorders: Current status and future directions. Clinical Psychology Review, 27, 384-405. doi:10.1016/j.cpr.2006.12.005 
Baumeister, R. F. (1997). Identity, self-concept, and self-esteem: The self lost and found. In R. Hogan, J. A. Johnson, \& S. R. Briggs (Eds.). Handbook of personality psychology. (pp. 681-710). San Diego, CA: Academic Press.

Baumeister, R. F., DeWall, C. N., Ciarocco, N. J., \& Twenge, J. M. (2005). Social exclusion impairs self-regulation. Journal of Personality and Social Psychology, 88, 589-604. doi:10.1037/0022-3514.88.4.589

Beaton, E. A., Schmidt, L. A., Ashbaugh, A. R., Santesso, D. L., \& Antony, M. M.... Schulkin, J. (2006). Low salivary cortisol levels among socially anxious young adults: Preliminary evidence from a selected and a non-selected sample. Personality and Individual Differences, 41, 1217-1228. doi:10.1016/j.paid.2006.02.020

Bieling, P. J., Israeli, A. L., \& Antony, M. M. (2004). Is perfectionism good, bad, or both? Examining models of the perfectionism construct. Personality and Individual Differences, 36, 1373-1385. doi:10.1016/S0191-8869(03)00235-6

Blechert, J., Wilhelm, F. H., Meuret, A. E., Wilhelm, E. M., \& Roth, W. T. (2010). Respiratory, autonomic, and experiential responses to repeated inhalations of $20 \% \mathrm{CO}_{2}$ enriched air in panic disorder, social phobia, and healthy controls. Biological Psychology, 84, 104-111. doi:10.1016/j.biopsycho.2010.01.002

Blonna, R. (2007). Coping with stress in a changing world (4th ed.). Boston, MA: McGraw-Hill.

Bohon, C., Stice, E., \& Burton, E. (2009). Maintenance factors for persistence of bulimic pathology: A prospective natural history study. International Journal of Eating Disorders, 42, 173-178. doi:10.1002/eat.20600 
Brewerton, T. D., Lydiard, R. B., Herzog, D. B., Brotman, A. W., O'Neil, P. M., \& Ballenger, J. C. (1995). Comorbidity of Axis I psychiatric disorders in bulimia nervosa. Journal of Clinical Psychiatry, 56, 77-80.

Buckner, J. D., Silgado, J., \& Lewinsohn, P. M. (2010). Delineation of differential temporal relations between specific eating and anxiety disorders. Journal of Psychiatric Research, 44, 781-787. doi:10.1016/j.jpsychires.2010.01.014

Bulik, C. M., Sullivan, P. F., Fear, J. L., \& Joyce, P. R. (1997). Eating disorders and antecedent anxiety disorders: A controlled study. Acta Psychiatrica Scandinavica, 96, 101-107. doi:10.1111/j.1600-0447.1997.tb09913.x

Caldirola, D., Perna, G., Arancio, C., Bertani, A., \& Bellodi, L. (1997). The $35 \% \mathrm{CO}_{2}$ challenge test in patients with social phobia. Psychiatry Research, 71, 41-48. doi:10.1016/S01651781(97)00038-3

Campbell, J., \& Ehlert, U. (2012). Acute psychosocial stress: Does the emotional stress response correspond with physiological responses? Psychoneuroendocrinology, 37, 1111-1134. doi: 10.1016/j.psyneuen.2011.12.010

Carroll, D., Phillips, A. C., \& Der, G. (2008). Body mass index, abdominal adiposity, obesity, and cardiovascular reactions to psychological stress in a large community sample. Psychosomatic Medicine, 20, 653-660.

Cash, T. F., \& Labarge, A. S. (1996). Development of the Appearance Schemas Inventory: A new cognitive body-image assessment. Cognitive Therapy and Research, 20, 37-50. doi:10.1007/BF02229242 
Cash, T. F., \& Szymanski, M. L. (1995). The development and validation of the Body-Image Ideals Questionnaire. Journal of Personality Assessment, 64, 466-477. doi:10.1207/s15327752jpa6403_6

Casper, R. C. (1998). Depression and eating disorders. Depression and Anxiety, 8 Suppl 1, 96104.

Castro-Fornieles, J., Gual, P., Lahortiga, F., Gila, A., Casula, V., Fuhrmann, C., . . Toro, J. (2007). Self-oriented perfectionism in eating disorders. International Journal of Eating Disorders, 40, 562-568. doi:10.1002/eat.20393

Cattanach, L. M., Malley, R., \& Rodin, J. (1988). Psychologic and physiologic reactivity to stressors in eating disordered individuals. Psychosomatic Medicine, 50, 591-599.

Cattanach, L. M., \& Rodin, J. (1988). Psychosocial components of the stress process in bulimia. International Journal of Eating Disorders, 7, 75-88.

Choi, K., Vickers, K., \& Tassone, A. (2014). Trait emotional intelligence, anxiety sensitivity, and experiential avoidance in stress reactivity and their improvement through psychological methods. Europe's Journal of Psychology, 10, 376-404. doi:10.5964/ejop.v10i2.754

Chopra, K. K., Ravindran, A., Kennedy, S. H., Mackenzie, B., Matthews, S., Anisman, H., . . . Levitan, R. D. (2009). Sex differences in hormonal responses to a social stressor in chronic major depression. Psychoneuroendocrinology, 34, 1235-1241. doi:10.1016/j.psyneuen.2009.03.014

Christenfeld, N. J. S., Sloan, R. P., Carroll, D., \& Greenland, S. (2004). Risk factors, confounding, and the illusion of statistical control. Psychosomatic Medicine, 66, 868-875. 
Claes, L., Hart, T. A., Smits, D., Van den Eynde, F., Mueller, A., \& Mitchell, J. E. (2012). Validation of the social appearance anxiety scale in female eating disorder patients. European Eating Disorders Review, 20, 406-409. doi:10.1002/erv.1147

Clark, D. M., \& Wells, A. (1995). A cognitive model of social phobia. In R. G. Heimberg, M. R. Liebowitz, D. A. Hope, \& F. R. Schneier (Eds.), Social phobia: Diagnosis, assessment, and treatment. New York, NY: Guilford Press.

Clark, D. M., \& Hemsley, D. R. (1982). The effects of hyperventilation: Individual variability and its relation to personality. Journal of Behavior Therapy and Experimental Psychiatry, 13, 41-47. doi:10.1016/0005-7916(82)90034-9

Cohen, J. (1988). Set correlation and contingency tables. Applied Psychological Measurement, 12, 425-434. 10.1177/014662168801200410

Cohen, J. (1992). A power primer. Psychological Bulletin, 112, 155-159. doi:http://dx.doi.org/10.1037/0033-2909.112.1.155

Cohen, S., \& Williamson, G. (1988). Perceived stress in a probability sample of the United States. In S. Spacaman, \& S. Oskamp (Eds.), The social psychology of health: Claremont Symposium on applied social psychology. Thousand Oaks, CA: Sage.

Cohen, S., Kamarck, T., \& Mermelstein, R. (1983). A global measure of perceived stress. Journal of Health and Social Behavior, 24, 385-396. doi:10.2307/2136404

Colasanti, A., Salamon, E., Schruers, K., van Diest, R., van Duinen, M., \& Griez, E. J. (2008). Carbon dioxide-induced emotion and respiratory symptoms in healthy volunteers. Neuropsychopharmacology, 33, 3103-3110. doi:10.1038/npp.2008.31 
Collins, K. A., Westra, H. A., Dozois, D. J., \& Stewart, S. H. (2005). The validity of the brief version of the Fear of Negative Evaluation Scale. Journal of Anxiety Disorders, 19, 345359. doi:10.1016/j.janxdis.2004.02.003

Connor, K. M., Davidson, J. R., Churchill, L. E., Sherwood, A., Foa, E., \& Weisler, R. H. (2000). Psychometric properties of the Social Phobia Inventory (SPIN). New self-rating scale. British Journal of Psychiatry, 176, 379-386. doi:10.1192/bjp.176.4.379

Cox, B. J., Parker, J. D., \& Swinson, R. P. (1996). Anxiety sensitivity: Confirmatory evidence for a multidimensional construct. Behaviour Research and Therapy, 34, 591-598. doi:10.1016/0005-7967(96)00006-X

Crow, S. J., Peterson, C. B., Swanson, S. A., Raymond, N. C., Specker, S., Eckert, E. D., \& Mitchell, J. E. (2009). Increased mortality in bulimia nervosa and other eating disorders. American Journal of Psychiatry, 166, 1342-1346. doi:10.1176/appi.ajp.2009.09020247

Crowther, J. H., \& Chernyk, B. (1986). Bulimia and binge eating in adolescent females: A comparison. Addictive Behaviors, 11, 415-424. doi:10.1016/0306-4603(86)90020-1

Crowther, J. H., Snaftner, J., Bonifazi, D. Z., \& Shepherd, K. L. (2001). The role of daily hassles in binge eating. International Journal of Eating Disorders, 29, 449-454. doi:10.1002/eat.1041

Davis, R., Freeman, R. J., \& Garner, D. M. (1988). A naturalistic investigation of eating behavior in bulimia nervosa. Journal of Consulting and Clinical Psychology, 56, 273-279. doi:10.1037/0022-006X.56.2.27.

Davis, C., Katzman, D. K., Kaptein, S., Kirsch, C., Brewer, H., Kalmbach, K., Olmsted, M. P., Woodside, D. B., Kaplan A. S., 1997. The prevalence of high level exercising in the eating disorders: Etiological implications. Comprehensive Psychiatry, 38, 321-236. 
Deacon, B., \& Abramowitz, J. (2006). Anxiety sensitivity and its dimensions across the anxiety disorders. Journal of Anxiety Disorders, 20, 837-857. doi:10.1016/j.janxdis.2006.01.003

Deacon, B. J., Abramowitz, J. S., Woods, C. M., \& Tolin, D. F. (2003). The Anxiety Sensitivity Index - Revised: Psychometric properties and factor structure in two nonclinical samples. Behaviour Research and Therapy, 41, 1427-1449. doi:10.1016/S0005-7967(03)00065-2

Deaver, C. M., Miltenberger, R. G., Smyth, J., Meidinger, A., \& Crosby, R. (2003). An evaluation of affect and binge eating. Behavior Modification, 27, 578-599.

Deboer, L. B., Medina, J. L., Davis, M. L., Presnell, K. E., Powers, M. B., \& Smits, J. A. (2013). Associations between fear of negative evaluation and eating pathology during intervention and 12-month follow-up. Cognitive Therapy and Research, 37, 941-952. doi:10.1007/s10608-013-9547-y

Deep, A. L., Nagy, L. M., Weltzin, T. E., Rao, R., \& Kaye, W. H. (1995). Premorbid onset of psychopathology in long-term recovered anorexia nervosa. International Journal of Eating Disorders, 17, 291-297.

DeMoja, C. A., \& DeMoja, G. (1986). State-trait anxiety and motocross performance. Perceptual and Motor Skills, 62, 107-110.

Deuter, C. E., Kuehl, L. K., Blumenthal, T. D., Schulz, A., Oitzl, M. S., \& Schachinger, H. (2012). Effects of cold pressor stress on the human startle response. PLOS ONE, 7, e49866. doi:10.1371/journal.pone.0049866

DiBartolo, P. M., \& Frost, R. O. (2008). How do the dimensions of perfectionism relate to mental health? Cognitive Therapy and Research, 32, 401-417. doi:10.1007/s10608-007$9157-7$ 
DiBartolo, P. M., Frost, R. O., Chang, P., Lasota, M., \& Grills, A. E. (2004). Shedding light on the relationship between personal standards and psychopathology. The case for contingent self-worth. Journal of Rational-Emotive and Cognitive-Behavior Therapy, 22, 237-250. doi:10.1023/B:JORE.0000047310.94044.ac

Dickerson, S. S., \& Kemeny, M. E. (2004). Acute stressors and cortisol responses: A theoretical integration and synthesis of laboratory research. Psychological Bulletin, 130, 355-391. doi:10.1037/0033-2909.130.3.355

Dienes, K. A., Hazel, N. A., \& Hammen, C. L. (2013). Cortisol secretion in depressed, and atrisk adults. Psychoneuroendocrinology, 38, 927-940. doi:10.1016/j.psyneuen.2012.09.019

Ditzen, B., Schmidt, S., Strauss, B., Nater, U. M., Ehlert, U., \& Heinrichs, M. (2008). Adult attachment and social support interact to reduce psychological but not cortisol responses to stress. Journal of Psychosomatic Research, 64, 479-486.

doi:10.1016/j.jpsychores.2007.11.011

Egan, S. J., Wade, T. D., \& Shafran, R. (2011). Perfectionism as a transdiagnostic process: A clinical review. Clinical Psychology Review, 31, 203-212. doi: 10.1016/j.cpr.2010.04.009

Eifert, G. H., Zvolensky, M. J., Sorrell, J. T., Hopko, D. R., \& Lejuez, C. W. (1999). Predictors of self-reported anxiety and panic symptoms: An evaluation of anxiety sensitivity, suffocation fear, heart-focused anxiety, and breath-holding duration. Journal of Psychopathology and Behavioral Assessment, 21, 293-305.

doi:10.1023/A:1022116731279 
Eisenberger, N. I., Taylor, S. E., Gable, S. L., Hilmert, C. J., \& Lieberman, M. D. (2007). Neural pathways link social support to attenuated neuroendocrine stress responses. Neuroimage, 35, 1601-1612. doi:10.1016/j.neuroimage.2007.01.038

Enders, C. K., \& Gottschall, A. C. (2011). Multiple imputation strategies for multiple group structural equation models. Structural Equation Modeling, 18, 35-54.

Epel, E., Lapidus, R., McEwen, B., \& Brownell, K. (2001). Stress may add bite to appetite in women: A laboratory study of stress-induced cortisol and eating behavior. Psychoneuroendocrinology, 26, 37-49. doi:10.1016/S0306-4530(00)00035-4

Fairburn, C. G. (1997). Towards evidence-based and cost-effective treatment for bulimia nervosa. European Eating Disorders Review,5, 145-148. doi:10.1002/(SICI)10990968(199709)5:3<145::AID-ERV206>3.0.CO;2-3

Fairburn, C. G. (2008). Cognitive behavior therapy and eating disorders. New York: The Gilford Press.

Fairburn, C. G., Cooper, Z., \& Cooper, P. J. (1986). The clinical features and maintenance of bulimia nervosa. In K. D. Brownell \& J. P. Foreyt (Eds.), Handbook of eating disorders: Physiology, psychology and treatment of obesity, anorexia and bulimia (pp. 389-404). New York: Basic Books.

Fairburn, C. G., Cooper, Z., Doll, H. A., Norman, P., \& O'Connor, M. (2000). The natural course of bulimia nervosa and binge eating disorder in young women. Archives of General Psychiatry, 57, 659-665. doi:10.1001/archpsyc.57.7.659

Fairburn, C. G., Cooper, Z., \& Shafran, R. (2003). Cognitive behaviour therapy for eating disorders: A "transdiagnostic" theory and treatment. Behaviour Research and Therapy, 41, 509-528. doi:10.1016/S0005-7967(02)00088-8 
Fairburn, C. G., \& Harrison, P. J. (2003). Eating disorders. Lancet, 361, 407-416. doi:10.1016/S0140-6736(03)12378-1

Federoff, I. C., Polivy, J., \& Herman, C. P. (1997). The effect of preexposure to food cues on the eating behavior of restrained and unrestrained eaters. Appetite, 28, 33-47.

Federoff, I. C., Polivy, J., \& Herman, C. P. (2003). The specificity of restrained versus unrestrained eaters' responses to food cues: general desire to eat, or craving for the cued food? Appetite, 41, 7-13.

Ferguson, C. J. (2009). An effect size primer: A guide for clinicians and researchers. Professional Psychology Research and Practice, 40, 532-538. doi:10.1037/a0015808

Fichter, M. M., \& Quadflieg, N. (2007). Long-term stability of eating disorder diagnoses. International Journal of Eating Disorders, 40 Suppl, S61-66. doi: 10.1002/eat.20443

First, M. B., Spitzer, R. L., Gibbon M., \& Williams, J. B. W. (2001) Structured Clinical Interview for DSM-IV-TR Axis I Disorders, Research Version, Patient Edition. (SCIDI/ P) New York: Biometrics Research, New York State Psychiatric Institute.

Foley, P., \& Kirschbaum, C. (2010). Human hypothalamus-pituitary-adrenal axis responses to acute psychosocial stress in laboratory settings. Neuroscience and Biobehavioral Reviews, 35, 91-96. doi:10.1016/j.neubiorev.2010.01.010

Frost, R. O., Heimberg, R. G., Holt, C. S., Mattia, J. L., \& Neubauer, A. L. (1993). A comparison of two measures of perfectionism. Personality and Individual Differences, 14, 119-126. doi:10.1016/0191-8869(93)90181-2

Frost, R. O., Marten, P., Lahart, C., \& Rosenblate, R. (1990). The dimensions of perfectionism. Cognitive Therapy and Research, 14, 449-468. doi:10.1007/BF01172967 
Garfinkel, P. E., Lin, E., Goering, P., Spegg, C., Goldbloom, D. S., Kennedy, S., . . Woodside, D. B. (1996). Purging and nonpurging forms of bulimia nervosa in a community sample. International Journal of Eating Disorders, 20, 231-238. doi:10.1002/(SICI)1098108X(199611)20:3

Garner, D. M., \& Garfinkel, P. E. (1979). The eating attitudes test: An index of the symptoms of anorexia nervosa. Psychological Medicine, 9, 273-279. 10.1017/S0033291700030762

Garner, D. M., Olmsted, M. P., Bohr, Y., \& Garfinkel, P. E. (1982). The eating attitudes test: Psychometric features and clinical correlates. Psychological Medicine, 12(4), 871878. doi:10.1017/S0033291700049163

Garner, D. M., Olmstead, M. A., \& Polivy, J. (1983). Development and validation of a multidimensional eating disorder inventory for anorexia nervosa and bulimia. International Journal of Eating Disorders, 2, 15-34.

Geller, J., Cockell, S. J., Hewitt, P. L., Goldner, E. M., \& Flett, G. L. (2000). Inhibited expression of negative emotions and interpersonal orientation in anorexia nervosa. International Journal of Eating Disorders, 28, 8-19. doi:10.1002/1098$108 X(200007) 28: 1$

Gerra, G., Zaimovic, A., Mascetti, G. G., Gardini, S., Zambelli, U., Timpano, M., . . Brambilla, F. (2001). Neuroendocrine responses to experimentally-induced psychological stress in healthy humans. Psychoneuroendocrinology, 26, 91-107. doi:10.1016/S0306$4530(00) 00046-9$

Gilbert, N., \& Meyer, C. (2003). Social anxiety and social comparison: Differential links with restrictive and bulimic attitudes among nonclinical women. Eating Behaviors, 4, 257264. doi:10.1016/S1471-0153(03)00026-6 
Gilbert, N., \& Meyer, C. (2005a). Fear of negative evaluation and eating attitudes: A replication and extension study. International Journal of Eating Disorders, 37, 360-363. doi:10.1002/eat.20103

Gilbert, N., \& Meyer, C. (2005b). Fear of negative evaluation and the development of eating psychopathology: A longitudinal study among nonclinical women. International Journal of Eating Disorders, 37, 307-312. doi:10.1002/eat.20105

Ginty, A. T., Phillips, A. C., Higgs, S., Heaney, J. L., \& Carroll, D. (2012). Disordered eating behaviour is associated with blunted cortisol and cardiovascular reactions to acute psychological stress. Psychoneuroendocrinology, 37, 715-724. doi:10.1016/j.psyneuen.2011.09.004

Gleaves, D. H., Pearson, C. A., Ambwani, \& Morey, L. C. (2014). Measuring eating disorder attitudes and behaviors: a reliability generalization study. Journal of Eating Disorders, , 2, 6. doi:10.1186/2050-2974-2-6.

Godart, N. T., Flament, M. F., Curt, F., Perdereau, F., Lang, F., Venisse, J. L., . . Fermanian, J. (2003). Anxiety disorders in subjects seeking treatment for eating disorders: A DSM-IV controlled study. Psychiatry Research, 117, 245-258. doi:10.1016/S01651781(03)00038-6

Godart, N. T., Flament, M. F., Lecrubier, Y., \& Jeammet, P. (2000). Anxiety disorders in anorexia nervosa and bulimia nervosa: Co-morbidity and chronology of appearance. European Psychiatry, 15, 38-45. doi:10.1016/S0924-9338(00)00212-1

Godart, N. T., Flament, M. F., Perdereau, F., \& Jeammet, P. (2002). Comorbidity between eating disorders and anxiety disorders: A review. International Journal of Eating Disorders, 32, 253-270. doi:10.1002/eat.10096 
Goldschmidt, A. B., Wonderlich, S. A., Crosby, R. D., Engel, S. G., Lavender, J. M., Peterson, C. B., . . Mitchell, J. E. (2014). Ecological momentary assessment of stressful events and negative affect in bulimia nervosa. Journal of Consulting and Clinical Psychology, 82, 30-39. doi:10.1037/a0034974

Goodwin, R. D., \& Fitzgibbon, M. L. (2002). Social anxiety as a barrier to treatment for eating disorders. International Journal of Eating Disorders, 32, 103-106. doi:10.1002/eat.10051

Gorman, J. M., Papp, L. A., Martinez, J., Goetz, R. R., Hollander, E., Liebowitz, M. R., \& Jordan, F. (1990). High-dose carbon dioxide challenge test in anxiety disorder patients. Biological Psychiatry, 28, 743-757. doi:10.1016/0006-3223(90)90510-9

Grabhorn, R., Stenner, H., Stangier, U., \& Kaufhold, J. (2006). Social anxiety in anorexia and bulimia nervosa: The mediating role of shame. Clinical Psychology and Psychotherapy, 13, 12-19. doi:10.1002/cpp.463

Greeno, C. G., \& Wing, R. R. (1994). Stress-induced eating. Psychological Bulletin, 115, 444464. Griez, E., de Loof, C., Pols, H., Zandbergen, J., \& Lousberg, H. (1990). Specific sensitivity of patients with panic attacks to carbon dioxide inhalation. Psychiatry Research, 31, 193-199. doi:10.1016/0165-1781(90)90121-K

Greeno, C. G., Wing, R. R., \& Shiffman, S. (2000). Binge antecedents in obese women with and without binge eating disorder. Journal of Consulting and Clinical Psychology, 68, 95102. doi:10.1037/0022-006X.68.1.95

Griez, E., \& Schruers, K. (1998). Experimental pathophysiology of panic. Journal of Psychosomatic Research, 45, 493-503. doi:10.1016/S0022-3999(98)00027-0

Griez, E., \& Schruers, K. (2003). Mechanisms of $\mathrm{CO}_{2}$ challenges. Journal of Psychopharmacology, 17, 260-262. doi:10.1177/02698811030173003 
Griez, E. J., Lousberg, H., van den Hout, M. A., \& van der Molen, G. M. (1987). CO 2 vulnerability in panic disorder. Psychiatry Research, 20, 87-95. doi:10.1016/01651781(87)90001-1

Grillon, C., Ameli, R., Foot, M., \& Davis, M. (1993). Fear-potentiated startle: Relationship to the level of state/trait anxiety in healthy subjects. Biological Psychiatry, 33, 566-574. doi:10.1016/0006-3223(93)90094-T

Grilo, C. M., Masheb, R. M., \& Berman, R. M. (2001). Subtyping women with bulimia nervosa along dietary and negative affect dimensions: A replication in a treatment-seeking sample. Eating and Weight Disorders, 6, 53-58. doi:10.1007/BF03339753

Gros, D. F., Antony, M. M., Simms, L. J., \& McCabe, R. E. (2007). Psychometric properties of the State-Trait Inventory for Cognitive and Somatic Anxiety (STICSA): Comparison to the State-Trait Anxiety Inventory (STAI). Psychological Assessment, 19, 369-381. doi:10.1037/1040-3590.19.4.369

Grossman, P., Wilhelm, F. H., Kawachi, I., \& Sparrow, D. (2001). Gender differences in psychophysiological responses to speech stress among older social phobics: Congruence and incongruence between self-evaluative and cardiovascular reactions. Psychosomatic Medicine, 63, 765-777.

Haikal, M., \& Hong, R. Y. (2010). The effects of social evaluation and looming threat on selfattentional biases and social anxiety. Journal of Anxiety Disorders, 24, 345-352. doi:10.1016/j.janxdis.2010.01.007

Hammen, C. (1991). Generation of stress in the course of unipolar depression. Journal of Abnormal Psychology, 100, 555-561. 
Harrison, W. M., Sandberg, D., Gorman, J. M., Fyer, M., Nee, J., Uy, J., \& Endicott, J. (1989). Provocation of panic with carbon dioxide inhalation in patients with premenstrual dysphoria. Psychiatry Research, 27, 183-192. doi:10.1016/0165-1781(89)90133-9

Hart, E. A., Leary, M. R., \& Rejeski, W. J. (1989). The measurement of social physique anxiety. Journal of Sport and Exercise Psychology, 11, 94-104. doi:10.13072/midss.598

Hart, T. A., Flora, D. B., Palyo, S. A., Fresco, D. M., Holle, C., \& Heimberg, R. G. (2008). Development and examination of the Social Appearance Anxiety Scale. Assessment, 15, 48-59. doi:10.1177/1073191107306673

Hawkins, R. C., \& Clement, P. F. (1984). Binge eating: Measurement problems and a conceptual model. In R. C. Hawkins, J. Fremouw, \& P. F. Clement (Eds.), The binge purge syndrome: Diagnosis, treatment and research (pp. 229-251). New York, NY: Springer.

Hearon, B. A., Utschig, A. C., Smits, J. A. J., Moshier, S. J., \& Otto, M. W. (2013). The role of anxiety sensitivity and eating expectancy in maladaptive eating behavior. Cognitive Therapy and Research, 37, 923-933. doi:10.1007/s10608-012-9491-2

Heatherton, T. F., \& Baumeister, R. F. (1991). Binge eating as escape from self-awareness. Psychological Bulletin, 110, 86-108. doi:10.1037/0033-2909.110.1.86

Heatherton, T. F., Herman, C. P., \& Polivy, J. (1991). Effects of physical and ego threat on eating behavior. Journal of Personality and Social Psychology, 60, 138-143. doi:10.1037/0022-3514.60.1.138.

Heimberg, R. G., Juster, H. R., Hope, D. A., \& Mattia, J. I. (1995). Cognitive behavioral group treatment: Description, case presentation, and empirical support. In M. B. Stein (Ed.), Social phobia: Clinical and research perspectives (pp. 293-321). Washington, DC: American Psychiatric Press. 
Henry, J. D., \& Crawford, J. R. (2005). The short-form version of the Depression Anxiety Stress Scales (DASS-21): Construct validity and normative data in a large non-clinical sample. British Journal of Clinical Psychology, 44, 227-239. doi:10.1348/014466505X29657

Herzog, D. B., Dorer, D. J., Keel, P. K., Selwyn, S. E., Ekeblad, E. R., Flores, A. T., . . Keller, M. B. (1999). Recovery and relapse in anorexia and bulimia nervosa: A 7.5-year followup study. Journal of the American Academy of Child and Adolescent Psychiatry, 38, 829837. doi:10.1097/00004583-199907000-00012

Het, S., Vocks, S., Wolf, J. M., Hammelstein, P., Herpertz, S., \& Wolf, O. T. (2015). Blunted neuroendocrine stress reactivity in young women with eating disorders. Journal of Psychosomatic Research, 78, 260-267. doi:10.1016/j.jpsychores.2014.11.001

Hewig, J., Hagermann, D., Seifert, J., Gollwitzer, M., Naumann, E., \& Bartussek, D. (2005). A revised film set for the induction of basic emotions. Cognition and Emotion, 19, 10951109. doi:10.1080/02699930541000084

Hewitt, P. L., Flett, G. L., Turnbull-Donovan, W., \& Mikail, S. F. (1991). The Multidimensional Perfectionism Scale. Reliability, validity, and psychometric properties in psychiatric samples. Psychological Assessment, 3, 464. doi:10.1037/1040-3590.3.3.464

Hilbert, A., Vögele, C., Tuschen-Caffier, B., \& Hartmann, A. S. (2011). Psychophysiological responses to idiosyncratic stress in bulimia nervosa and binge eating disorder. Physiology \& Behavior, 104, 770-777. doi:10.1016/j.physbeh.2011.07.013

Hinrichsen, H., Wright, F., Waller, G., \& Meyer, C. (2003). Social anxiety and coping strategies in the eating disorders. Eating Behaviors, 4, 117-126. doi:10.1016/S14710153(03)00016-3 
Hinz, L. D., \& Williamson, D. A. (1987). Bulimia and depression: A review of the affective variant hypothesis. Psychological Bulletin, 102, 150-158.

Hoek, H. W., \& van Hoeken, D. (2003). Review of the prevalence and incidence of eating disorders. International Journal of Eating Disorders, 34, 383-396. doi:10.1002/eat.10222

Hofmann, S. G. (2007). Cognitive factors that maintain social anxiety disorder: A comprehensive model and its treatment implications. Cognitive Behaviour Therapy, 36, 193-209. doi:10.1080/16506070701421313

Holmes, T. H., \& Rahe, R. H. (1967). The Social Readjustment Scale. Journal of Psychosomatic Research, 11, 213-218. doi:10.1016/0022-3999(67)90010-4

Holt, P. E., \& Andrews, G. (1989a). Hyperventilation and anxiety in panic disorder, social phobia, GAD and normal controls. Behaviour Research and Therapy, 27, 453-460. doi:10.1016/0005-7967(89)90016-8

Holt, P. E., \& Andrews, G. (1989b). Provocation of panic: Three elements of the panic reaction in four anxiety disorders. Behaviour Research and Therapy, 27, 253-261. doi:10.1016/0005-7967(89)90044-2

Hoyer, J., Braeuer, D., Crawcour, S., Klumbies, E., \& Kirschbaum, C. (2013). Depersonalization/derealization during acute social stress in social phobia. Journal of Anxiety Disorders, 27, 178-187. doi:10.1016/j.janxdis.2013.01.002

Hudson, J. I., Hiripi, E., Pope, H. G., Jr., \& Kessler, R. C. (2007). The prevalence and correlates of eating disorders in the National Comorbidity Survey Replication. Biological Psychiatry, 61, 348-358. doi:10.1016/j.biopsych.2006.03.040 
Hudson, J. I., Pope, H. G., Jr., Jonas, J. M., Laffer, P. S., Hudson, M. S., \& Melby, J. C. (1983). Hypothalamic-pituitary-adrenal-axis hyperactivity in bulimia. Psychiatry Research, 8, 111-117.

Hofmann, S. G., Newman, M. G., Ehlers, A., \& Roth, W. T. (1995). Psychophysiological differences between subgroups of social phobia. Journal of Abnormal Psychology, 104, 224-231.

Iwasaki, Y., Kriike, N., Matsunaga, H., Nagata, T., \& Yamagami, S. (1999). Comorbidity of anxiety disorders in patients with eating disorders. Clinical Psychiatry, 41, 855-859.

Jacobi, C., Hayward, C., de Zwaan, M., Kraemer, H. C., \& Agras, W. S. (2004). Coming to terms with risk factors for eating disorders: Application of risk terminology and suggestions for a general taxonomy. Psychological Bulletin, 130, 19-65. doi:10.1037/0033-2909.130.1.19

Jezova, D., Makatsori, A., Duncko, R., Moncek, F., \& Jakubek, M. (2004). High trait anxiety in healthy subjects is associated with low neuroendocrine activity during psychosocial stress. Progressive Neuropsychopharmacology and Biological Psychiatry, 28, 13311336. doi:10.1016/j.pnpbp.2004.08.005

Juster, H. R., Heimberg, R. G., \& Holt, C. S. (1996). Social phobia: Diagnostic issues and review of cognitive behavioral treatment strategies. Progress in Behavior Modification, 30, 7498.

Kashdan, T. B., Zvolensky, M. J., \& McLeish, A. C. (2008). Anxiety sensitivity and affect regulatory strategies: Individual and interactive risk factors for anxiety-related symptoms. Journal of Anxiety Disorders, 22, 429-440. doi:10.1016/j.janxdis.2007.03.011 
Kaye, J., Buchanan, F., Kendrick, A., Johnson, P., Lowry, C., Bailey, J., . . Lightman, S.

(2004). Acute carbon dioxide exposure in healthy adults: Evaluation of a novel means of investigating the stress response. Journal of Neuroendocrinology, 16, 256-264. doi:10.1111/j.0953-8194.2004.01158.x

Kaye, J., Soothill, P., Hunt, M., \& Lightman, S. (2004). Responses to the $35 \% \mathrm{CO}_{2}$ challenge in postpartum women. Clinical Endocrinology, 61, 582-588. doi:10.1111/j.13652265.2004.02133.x

Kaye, W. H., Bulik, C. M., Thornton, L., Barbarich, N., \& Masters, K. (2004). Comorbidity of anxiety disorders with anorexia and bulimia nervosa. American Journal of Psychiatry, 161, 2215-2221. doi:10.1176/appi.ajp.161.12.2215

Keel, P. K. (2013). Commentary on empirical examinations of the association between anxiety and eating disorders. Cognitive Therapy and Research, 37. doi:10.1007/s10608-0139566-8

Keel, P. K., Mitchell, J. E., Miller, K. B., Davis, T. L., \& Crow, S. J. (2000). Predictive validity of bulimia nervosa as a diagnostic category. American Journal of Psychiatry, 157, 136138.

Keller, A., Litzelman, K., Wisk, L. E., Maddox, T., Cheng, E. R., Creswell, P. D., \& Witt, W. P. (2012). Does the perception that stress affects health matter? The association with health and mortality. Health Psychology, 31, 677-684. doi:10.1037/a0026743

Kessler, R. C., Shahly, V., Hudson, J. I., Supina, D., Berglund, P. A., Chiu, W. T., . . Xavier, M. (2014). A comparative analysis of role attainment and impairment in binge-eating disorder and bulimia nervosa: Results from the WHO World Mental Health Surveys. Epidemiology and Psychiatric Sciences, 23, 27-41. doi:10.1017/S2045796013000516 
Kirschbaum, C., Pirke, K. M., \& Hellhammer, D. H. (1993). The 'Trier Social Stress Test'--a tool for investigating psychobiological stress responses in a laboratory setting. Neuropsychobiology, 28, 76-81. doi:10.1159/000119004

Koo-Loeb, J. J., Costello, N., Light, K.C., \& Girdler, S.S. (2000). Women with eating disorder tendencies display altered cardiovascular, neuroendocrine, and psychosocial profiles. Psychosomatic Medicine, 62, 539-548.

Koo-Loeb, J. H., Pedersen, C., \& Girdler, S. S. (1998). Blunted cardiovascular and catecholamine stress reactivity in women with bulimia nervosa. Psychiatry Research, 80, 13-27.

Koskina, A., Van den Eynde, F., Meisel, S., Campbell, I. C., \& Schmidt, U. (2011). Social appearance anxiety and bulimia nervosa. Eating and Weight Disorders, 16, e142-145. doi:10.1007/BF03325321

Krämer, M., Seefeldt, W. L., Heinrichs, N., Tuschen-Caffier, B., Schmitz, J., Wolf, O. T., \& Blechert, J. (2012). Subjective, autonomic, and endocrine reactivity during social stress in children with social phobia. Journal of Abnormal Child Psychology, 40, 95-104. doi:10.1007/s10802-011-9548-9

Kudielka, B. M., Hellhammer, D. H., \& Kirschbaum, C. . (2007). Ten years of research with the Trier Social Stress Test - revisited. In E. Harmon-Jones, Vinkielman, P. (Eds.), Social neuroscience: Integrating biological and psychological explanations of social behavior (pp. 56-83). New York, NY: Guilford Press.

Lazarus, R. S. (1993). From psychological stress to the emotions: A history of changing outlooks. Annual Review of Psychology, 44, 1-21. doi:10.1146/annurev.ps.44.020193.000245 
Lazarus, R. S., \& Folkman, S. (1984). Stress, appraisal, and coping. New York, NY: Springer.

Leary, M. R. (1983). A brief version of the fear of negative evlauation scale. Personality and Social Psychology Bulletin, 9, 371-375. doi:10.1177/0146167283093007

Levinson, C. A., \& Rodebaugh, T. L. (2011). Validation of the Social Appearance Anxiety Scale: Factor, convergent, and divergent validity. Assessment, 18, 350-356. doi:10.1177/1073191111404808

Levinson, C. A., \& Rodebaugh, T. L. (2012). Social anxiety and eating disorder comorbidity: The role of negative social evaluation fears. Eating Behaviors, 13, 27-35. doi:10.1016/j.eatbeh.2011.11.006

Levinson, C. A., Rodebaugh, T. L., White, E. K., Menatti, A. R., Weeks, J. W., Iacovino, J. M., \& Warren, C. S. (2013). Social appearance anxiety, perfectionism, and fear of negative evaluation: Distinct or shared risk factors for social anxiety and eating disorders? Appetite, 67, 125-133. doi:10.1016/j.appet.2013.04.002

Liebowitz, M. R. (1987). Social phobia. Modern Problems of Pharmacopsychiatry, 22, 141-173.

Liebowitz, M. R., Gorman, J. M., Fyer, A. J., Dillon, D. J., \& Klein, D. F. (1984). Effects of naloxone on patients with panic attacks. American Journal of Psychiatry, 141, 995-997.

Linett, A., Carney, C., Antony, M., \& Vickers, K. (2016). Piloting an eating disorder specific stressor: Does watching a body-related video clip provoke stress in individuals with abnormal eating behaviours? Manuscript in preparation.

Lo Sauro, S., Ravaldi C., Cabras, P. L., Faravelli, C., \& Ricca, V. (2008). Stress, hypothalamicpituitary-adrenal axis and eating disorders. Neuropsychobiology, 57, 95-115. 
Loft, P., Thomas, M. G., Petrie, K. J., Booth, R. J., Miles, J., \& Vedhara, K. (2007). Examination stress results in altered cardiovascular responses to acute challenge and lower cortisol. Psychoneuroendocrinology, 32, 367-375. doi:10.1016/j.psyneuen.2007.02.004

Lorenzen TJ, Anderson VL. (1993). Design of experiments: A no-name approach. New York, NY: Marcel Dekker.

Lovibond, P. F., \& Lovibond, S. H. (1995). The structure of negative emotional states: comparison of the Depression Anxiety Stress Scales (DASS) with the Beck Depression and Anxiety Inventories. Behaviour Research and Therapy, 33, 335-343. doi:10.1016/0005-7967(94)00075-U

Lundh, L. G., \& Öst, L. G. (2001). Attentional bias, self-consciousness and perfectionism in social phobia before and after cognitive-behaviour therapy. Scandinavian Journal of Behaviour Therapy, 30, 4-16. doi:10.1080/028457101300140428

Lundh, L. G., \& Öst, L. G. (1996). Recognition bias for critical faces in social phobics. Behaviour Research and Therapy, 34, 787-794. doi:10.1016/0005-7967(96)00035-6

Maruyama, Y., Kawano, A., Okamoto, S., Ando, T., Ishitobi, Y., Tanaka, Y., . . Akiyoshi, J. (2012). Differences in salivary alpha-amylase and cortisol responsiveness following exposure to electrical stimulation versus the Trier Social Stress Tests. PLOS ONE, 7, e39375. doi:10.1371/journal.pone.0039375

McEwen, B. S. (2007). Physiology and neurobiology of stress and adaptation: Central role of the brain. Physiological Reviews, 87, 873-904. doi:10.1152/physrev.00041.2006

McFarlane, T., Urbszat, D., \& Olmsted, M. P. (2011). "I feel fat": An experimental induction of body displacement in disordered eating. Behaviour Research and Therapy, 49, 289-293. doi:10.1016/j.brat.2011.01.008 
McLean, C. P., Miller, N. A., \& Hope, D. A. (2007). Mediating social anxiety and disordered eating: the role of expressive suppression. Eating Disorders, 15, 41-54. doi:10.1080/10640260601044485

McNally, R. J. (1994). Panic disorder: A critical analysis. New York, NY: Guilford Press. McNally, R. J. (2002). Anxiety sensitivity and panic disorder. Biological Psychiatry, 52, 938946. doi:10.1016/S0006-3223(02)01475-0

Mehler, P. S. (2011). Medical complications of bulimia nervosa and their treatments. International Journal of Eating Disorders, 44, 95-104. doi:10.1002/eat.20825

Menatti, A. R., Weeks, J. W., Levinson, C. A., \& McGowan, M. M. (2013). Exploring the relationship between social anxiety and bulimic symptoms: Mediational effects of perfectionism among females. Cognitive Therapy and Research, 37, 914-922. doi:10.1007/s10608-013-9521-8

Messerli-Burgy, N., Engesser, C., Lemmenmeier, E., Steptoe, A., \& Laederach-Hoffman, K. (2010). Cardiovascular stress reactivity and recovery in bulimia nervosa and binge eating disorder. International Journal of Psychophysiology, 78, 163-168

Mills, J. S., \& Miller, J. L. (2007). Experimental effects of receiving negative weight-related feedback: A weight guessing study. Body Image, 4, 309-316. doi:10.1016/j.bodyim.2007.04.004

Mitchell, J. E., Hoberman, H. N., Peterson, C. B., Mussell, M., \& Pyle, R. L. (1996). Research on the psychotherapy of bulimia nervosa: Half empty or half full. International Journal of Eating Disorders, 20, 219-229. doi:10.1002/(SICI)1098108X(199611)20:3

Mond, J.M., Hay, P.J., Rodgers, B., Owen, C., Crosby, R., Mitchell, J.E. (2006). Use of extreme weight control behaviors with and without binge eating in a community sample of 
women: Implications for the classification of bulimic-type eating disorders. International Journal of Eating Disorders, 39, 294-302. doi:10.1002/eat.20265.

Mond, J.M., Hay, P.J., Rodgers, B., Owen, C., Mitchell, J.E. (2006). Correlates of the use of purging and non-purging methods of weight control in a community sample of women. Australian and New Zealand Journal of Psychiatry, 40, 136-142. doi:10.1080/j.14401614.2006.01760.x.

Mond, J.M., Rodgers, B., Hay, P.J., Owen, C. (2011). Mental health impairment associated with eating-disordered behaviour in a community sample of women. Journal of Mental Health, 20, 456-66. doi:10.3109/09638237.2011.577845.

Monkul, E. S., Onur, E., Tural, U., Hatch, J. P., Alkin, T., Yucel, B., \& Fidaner, H. (2010). History of suffocation, state-trait anxiety, and anxiety sensitivity in predicting $35 \%$ carbon dioxide-induced panic. Psychiatry Research, 179, 194-197. doi:10.1016/j.psychres.2009.06.015

Monteleone, P., Tortorella, A., Scognamiglio, P., Serino, I., Monteleone, A. M., \& Maj, M. (2012). The acute salivary ghrelin response to a psychosocial stress is enhanced in symptomatic patients with bulimia nervosa: A pilot study. Neuropsychobiology, 66, 230236. doi:10.1159/000341877

Moscovitch, D. A. (2009). What is the core fear in social phobia? A new model to facilitate individualized case conceptualization and treatment. Cognitive and Behavioral Practice, 16, 123-134. doi:10.1016/j.cbpra.2008.04.002

Moscovitch, D. A., Orr, E., Rowa, K., Reimer, S. G., \& Antony, M. M. (2009). In the absence of rose-colored glasses: ratings of self-attributes and their differential certainty and 
importance across multiple dimensions in social phobia. Behaviour Research and Therapy, 47, 66-70. doi:10.1016/j.brat.2008.10.007

National Institute for Clinical Excellence. (2004). Eating disorders: Core interventions in the treatment and management of anorexia nervosa, bulimia nervosa, and related eating disorders. London, UK: National Collaborating Centre for Mental Health.

Norton, G. R., Rockman, G. E., Ediger, J., Pepe, C., Goldberg, S., Cox, B. J., \& Asmundson, G. J. (1997). Anxiety sensitivity and drug choice in individuals seeking treatment for substance abuse. Behaviour Research and Therapy, 35, 859-862. doi:10.1016/S00057967(97)00037-5

O'Brien, K. M., \& Vincent, N. K. (2003). Psychiatric comorbidity in anorexia and bulimia nervosa: Nature, prevalence, and causal relationships. Clinical Psychology Review, 23, 57-74. doi:10.1016/S0272-7358(02)00201-5

O'Connor, D. B., Jones, F., Conner, M., McMillan, B., \& Ferguson, E. (2008). Effects of daily hassles and eating style on eating behavior. Health Psychology, 27, S20-S31. doi:10.1037/0278-6133.27.1.S20

Oliver, G., Wardle, J., \& Gibson, E. L. (2000). Stress and food choice: A laboratory study. Psychosomatic Medicine, 62, 853-865.

Oliver, K. G., Huon, G. F., Zadro, L., \& Williams, K. D. (2001). The role of interpersonal stress in overeating among high and low disinhibitors. Eating Behaviors, 2, 19-26. doi:10.1016/S1471-0153(00)00020-9

Otto, M. W., Pollack, M. H., Fava, M., Uccello, R., \& Rosenbaum, J. F. (1995). Elevated Anxiety Sensitivity Index scores in patients with major depression: Correlates and 
changes with antidepressant treatment. Journal of Anxiety Disorders, 9, 117-123. doi:10.1016/0887-6185(94)00035-2

Pallister, E., \& Waller, G. (2008). Anxiety in the eating disorders: Understanding the overlap. Clinical Psychology Review, 28, 366-386. doi:10.1016/j.cpr.2007.07.001

Papp, L. A., Klein, D. F., Martinez, J., Schneier, F., Cole, R., Liebowitz, M. R., . . Gorman, J. M. (1993). Diagnostic and substance specificity of carbon-dioxide-induced panic. American Journal of Psychiatry, 150, 250-257.

Perna, G., Barbini, B., Cocchi, S., Bertani, A., \& Gasperini, M. (1995). 35\% $\mathrm{CO}_{2}$ challenge in panic and mood disorders. Journal of Affective Disorders, 33, 189-194. doi:10.1016/0165-0327(94)00088-Q

Perna, G., Battaglia, M., Garberi, A., Arancio, C., Bertani, A., \& Bellodi, L. (1994). Carbon dioxide/oxygen challenge test in panic disorder. Psychiatry Research, 52, 159-171. doi:10.1016/0165-1781(94)90085-X

Perna, G., Casolari, A., Bussi, R., Cucchi, M., Arancio, C., \& Bellodi, L. (2004). Comparison of $35 \%$ carbon dioxide reactivity between panic disorder and eating disorder. Psychiatry Research, 125, 277-283. doi:10.1016/j.psychres.2003.12.017

Perna, G., Gabriele, A., Caldirola, D., \& Bellodi, L. (1995). Hypersensitivity to inhalation of carbon dioxide and panic attacks. Psychiatry Research, 57, 267-273. doi:10.1016/01651781(95)02723-A

Perna, G., Romano, P., Caldirola, D., Cocchi, M., \& Bellodi, L. (2003). Anxiety sensitivity and $35 \% \mathrm{CO}_{2}$ reactivity in patients with panic disorder. Journal of Psychosomatic Research, 54, 573-577. doi:10.1016/S0022-3999(02)00468-3

Petrowski, K., Winterman, G., Shaarschmidt, M., Bornstein, S. R., \& Kirschbaum, C. (2013). 
Blunted salivary and plasma cortisol response in patients with panic disorder under psychosocial stress. International Journal of Psychophysiology, 88, 35-39.

Pirke, K. M., Platte, P., Laessle, R., Seidl, M., \& Fichter, M. M. (1992). The effect of a mental challenge test of plasma norepinephrine and cortisol in bulimia nervosa and in controls. Biological Psychiatry, 32, 202-206. doi:10.1016/0006-3223(92)90026-V

Polivy, J., \& Herman, C. P. (1985). Dieting and binging: A causal analysis. American Psychologist, 40, 193-201. doi:10.1037/0003-066X.40.2.193

Rapee, R. M., Brown, T. A., Antony, M. M., \& Barlow, D. H. (1992). Response to hyperventilation and inhalation of 5.5\% carbon dioxide-enriched air across the DSM-IIIR anxiety disorders. Journal of Abnormal Psychology, 101, 538-552. doi:10.1037/0021843X.101.3.538

Rapee, R. M., \& Heimberg, R. G. (1997). A cognitive-behavioral model of anxiety in social phobia. Behaviour Research and Therapy, 35, 741-756. doi:10.1016/S00057967(97)00022-3

Rassovsky, Y., \& Kushner, M. G. (2003). Carbon dioxide in the study of panic disorder: Issues of definition, methodology, and outcome. Journal of Anxiety Disorders, 17, 1-32. doi:10.1016/S0887-6185(02)00181-0

Rector, N. A., Szacun-Shimizu, K., \& Leybman, M. (2007). Anxiety sensitivity within the anxiety disorders: Disorder-specific sensitivities and depression comorbidity. Behaviour Research and Therapy, 45, 1967-1975. doi:10.1016/j.brat.2006.09.017

Ree, M. J., French, D., MacCleod, C., \& Locke, V. (2008). Distinguishing cognitive and somatic dimensions of state and trait anxiety: Development and validation of the State-Trait 
Inventory for Cognitive and Somatic Anxiety (STICSA). Behavioural and Cognitive Psychotherapy, 36, 313-332. doi:10.1017/S1352465808004232

Reinhardt, T., Schmahl, C., Wust, S., \& Bohus, M. (2012). Salivary cortisol, heart rate, electrodermal activity and subjective stress responses to the Mannheim Multicomponent Stress Test (MMST). Psychiatry Research, 198, 106-111.

doi:10.1016/j.psychres.2011.12.009

Reiss, S. (1991). Expectancy model of fear, anxiety, and panic. Clinical Psychology Review, 11, 141-153. doi:10.1016/0272-7358(91)90092-9

Reiss, S., \& McNally, R. J. (1985). Expectancy model of fear. In S. Reiss, \& R. R. Bootzin (Eds.), Theoretical issues in behavior therapy (pp. 107-121). New York, NY: Academic Press.

Reiss, S., Peterson, R. A., Gursky, D. M., \& McNally, R. J. (1986). Anxiety sensitivity, anxiety frequency and the prediction of fearfulness. Behaviour Research and Therapy, 24, 1-8. doi:10.1016/0005-7967(86)90143-9

Richardson, C. M., Rice, K. G., \& Devine, D. P. (2014). Perfectionism, emotion regulation, and the cortisol stress response. Journal of Counselling Psychology, 61, 110-118. doi:10.1037/a0034446

Rieger, E., Van Buren, D. J., Bishop, M., Tanofsky-Kraff, M., Welch, R., \& Wilfley, D. E. (2010). An eating disorder specific model of interpersonal psychotherapy (IPT-ED): Causal pathways and treatment implications. Clinical Psychology Review, 30, 400-410. doi:10.1016/j.cpr.2010.02.001 
Ruscio, A. M., Brown, T. A., Chiu, W. T., Sareen, J., Stein, M. B., \& Kessler, R. C. (2008). Social fears and social phobia in the USA: Results from the National Comorbidity Survey Replication. Psychological Medicine, 38, 15-28. doi:10.1017/S0033291707001699

Roelofs, K., van Peer, J., Berretty, E., Jong, P., Spinhoven, P., \& Elzinga, B. M. (2009). Hypothalamus-pituitary-adrenal axis hyperresponsiveness is associated with increased social avoidance behavior in social phobia. Biological Psychiatry, 65, 336-343. doi:10.1016/j.biopsych.2008.08.022

Ruderman, A. J. (1985). Dysphoric mood and overeating: A test of restraint theory's disinhibition hypothesis. Journal of Abnormal Psychology, 94, 78-85.

Sanderson, W. C., Rapee, R. M., \& Barlow, D. H. (1989). The influence of an illusion of control on panic attacks induced via inhalation of 5.5\% carbon dioxide-enriched air. Archives of General Psychiatry, 46, 157-162.

Schaefer, W. K., Maclennan, R. N., Yaholnitsky-Smith, S. A., \& Stover, E. D. (1998). Psychometric evaluation of the Eating Disorder Inventory (EDI) in a clinical group. Psychology and Health, 13, 873-881. doi:10.1080/08870449808407437

Schmidt, N. B., Lerew, D. R., \& Jackson, R. J. (1997). The role of anxiety sensitivity in the pathogenesis of panic: Prospective evaluation of spontaneous panic attacks during acute stress. Journal of Abnormal Psychology, 106, 355-364. doi:10.1037/0021843X.106.3.355

Schmidt, N. B., \& Richey, J. A. (2008). Social anxiety symptoms uniquely predict fear responding to $35 \% \mathrm{CO}_{2}$ challenge. Journal of Psychiatric Research, 42, 851-857. doi:10.1016/j.jpsychires.2007.08.010 
Schruers, K., Klaassen, T., Pols, H., Overbeek, T., Deutz, N. E., \& Griez, E. (2000). Effects of tryptophan depletion on carbon dioxide provoked panic in panic disorder patients. Psychiatry Research, 93, 179-187. doi:10.1016/S0165-1781(00)00117-7

Schutters, S. I., Viechtbauer, W., Knuts, I. J., Griez, E. J., \& Schruers, K. R. (2012). 35\% CO 2 sensitivity in social anxiety disorder. Journal of Psychopharmacology, 26, 479-486. doi:10.1177/0269881111430750

Schwabe, L., Haddad, L., \& Schachinger, H. (2008). HPA axis activation by a socially evaluated cold-pressor test. Psychoneuroendocrinology, 33, 890-895. doi:10.1016/j.psyneuen.2008.03.001

Schwalberg, M. D., Barlow, D. H., Alger, S. A., \& Howard, L. J. (1992). Comparison of bulimics, obese binge eaters, social phobics, and individuals with panic disorder on comorbidity across DSM-III-R anxiety disorders. Journal of Abnormal Psychology, 101, 675-681. doi:10.1037/0021-843X.101.4.675

Selye, H. (1950). Stress and the general adaptation syndrome. British Medical Journal, 13831392.

Shapiro, J. R., Berkman, N. D., Brownley, K. A., Sedway, J. A., Lohr, K. N., \& Bulik, C. M. (2007). Bulimia nervosa treatment: A systematic review of randomized controlled trials. International Journal of Eating Disorders, 40, 321-336. doi:10.1002/eat.20372

Sheehan. D. V. (2014). The Mini-International Neuropsychiatric Interview, Version 7.0 for DSM-5 (M.I.N.I. 7.0). Medical Outcomes Systems.

Sheehan, D., Lecrubier, Y., Sheehan, K., Amorim, P., Janavs, J., Weiller, E., et al., (1998). The Mini-International Neuropsychiatric Interview (M.I.N.I.): The development and validation of a structured diagnostic psychiatric interview for DSM-IV and ICD-10. 
Journal of Clinical Psychiatry, 59, 22-33.

Shepherd, H., \& Ricciardelli, L. A. (1998). Test of Stice's dual pathway model: Dietary restraint and negative affect as mediators of bulimic behavior. Behaviour Research and Therapy, 36(3), 345-352. doi:10.1016/S0005-7967(98)00033-3

Shufflebotham, J., Wetherell, M. A., Hince, D., Hood, S., Lightman, S., Nutt, D., . . Potokar, J. (2009). Women with diarrhoea-predominant irritable bowel syndrome show an increased pressure response to $35 \%$ carbon dioxide stress challenge. Stress, 12, 30-36. doi:10.1080/10253890801976926

Silgado, J., Timpano, K. R., Buckner, J. D., \& Schmidt, N. B. (2010). Social anxiety and bulimic behaviors: The moderating role of perfectionism. Cognitive Therapy and Research, 34, 487-492. doi:10.1007/s10608-009-9278-2

Simeon, D. (2004). Depersonalization disorder: a contemporary overview. CNS Drugs, 18, 343354. doi:10.2165/00023210-200418060-00002

Skoluda, N., Strahler, J., Schlotz, W., Niederberger, L., Marques, S., Fischer, S. Nater, U. M. (2015). Intra-individual psychological and physiological responses to acute laboratory stressors of different intensity. Psychoneuroendocrinology, 51, 227-236. doi:10.1016/j.psyneuen.2014.10.002

Smyth, J. M., Wonderlich, S. A., Heron, K. E., Sliwinski, M. J., Crosby, R. D., Mitchell, J. E., \& Engel, S. G. (2007). Daily and momentary mood and stress are associated with binge eating and vomiting in bulimia nervosa patients in the natural environment. Journal of Consulting and Clinical Psychology, 75, 629-638. doi:10.1037/0022-006X.75.4.629 
Spector, I. P., Pecknold, J. C., \& Libman, E. (2003). Selective attentional bias related to the noticeability aspect of anxiety symptoms in generalized social phobia. Journal of Anxiety Disorders, 17, 517-531. doi:10.1016/S0887-6185(02)00232-3

Spielberger, C. D. (1966). Theory and research on anxiety. In C. D. Spielberger (Ed.), Anxiety and behavior (pp. 3-19). New York, NY: Academic Press.

Spielberger, C. D. (1983). Manual for the State-Trait Anxiety Inventory (Form Y). Palo Alto, CA: Mind Garden.

Steele, A., Corsini, N., \& Wade, T. D. (2007). The interaction of perfectionism, perceived weight status, and self-esteem to predict bulimic symptoms: The role of 'benign' perfectionism. Behaviour Research and Therapy, 45, 1647-1655. doi:10.1016/j.brat.2006.08.007

Steketee, G., \& Chambless, D. L. (1992). Methodological issues in prediction of treatment outcome. Clinical Psychology Review, 12(4), 387-400. doi:10.1016/02727358(92)90123-P

Stice, E. (2001). A prospective test of the dual-pathway model of bulimic pathology: Mediating effects of dieting and negative affect. Journal of Abnormal Psychology, 110, 124-135. doi:10.1037/0021-843X.110.1.124

Stice, E. (2002). Risk and maintenance factors for eating pathology: A meta-analytic review. Psychological Bulletin, 128, 825-848. doi:10.1037/0033-2909.128.5.825

Stice, E., \& Agras, W.S. (1998). Predicting onset and cessation of bulimic behaviors during adolescence: A longitudinal grouping analysis. Behavior Therapy, 29, 257-276. doi:10.1016/S0005-7894(98)80006-3 
Stice, E., \& Agras, W. S. (1999). Subtyping bulimic women along dietary restraint and negative affect dimensions. Journal of Consulting and Clinical Psychology, 67, 460-469. doi:10.1037/0022-006X.67.4.460

Stice, E., Bohon, C., Marti, C. N., \& Fischer, K. (2008). Subtyping women with bulimia nervosa along dietary and negative affect dimensions: Further evidence of reliability and validity. Journal of Consulting and Clinical Psychology, 76, 1022-1033. doi:10.1037/a0013887

Stice, E., \& Fairburn, C. G. (2003). Dietary and dietary-depressive subtypes of bulimia nervosa show differential symptom presentation, social impairment, comorbidity, and course of illness. Journal of Consulting and Clinical Psychology, 71, 1090-1094. doi:10.1037/0022-006X.71.6.1090

Stice, E., Marti, C. N., \& Rohde, P. (2013). Prevalence, incidence, impairment, and course of the proposed DSM-5 eating disorder diagnoses in an 8-year prospective community study of young women. Journal of Abnormal Psychology, 122, 445-457. doi:10.1037/a0030679

Stice, E., Marti, C. N., Shaw, H., \& Jaconis, M. (2009). An 8-year longitudinal study of the natural history of threshold, subthreshold, and partial eating disorders from a community sample of adolescents. Journal of Abnormal Psychology, 118, 587-597. doi:10.1037/a0016481

Stice, E., Shaw, H. E., \& Nemeroff, C. (1998). Dual pathway model of bulimia nervosa: Longitudinal support for dietary restraint and affect-regulation mechanisms. Journal of Social and Clinical Psychology, 17, 129-149. doi:10.1521/jscp.1998.17.2.129

Stoeber, J., Harris, R. A., \& Moon, P. S. (2007). Perfectionism and the experience of pride, shame, and guilt: Comparing healthy perfectionists, unhealthy perfectionists, and non- 
perfectionists. Personality and Individual Differences, 43, 131-141.

doi:10.1016/j.paid.2006.11.012

Striegel-Moore, R. H., Silberstein, L. R., \& Rodin, J. (1986). Toward an understanding of risk factors for bulimia. American Psychologist, 41, 246-263. doi:10.1037/0003066X.41.3.246

Striegel-Moore, R. H., Silberstein, L. R., \& Rodin, J. (1993). The social self in bulimia nervosa: public self-consciousness, social anxiety, and perceived fraudulence. Journal of Abnormal Psychology, 102, 297-303. doi:10.1037/0021-843X.102.2.297

Svaldi, J., Caffier, D., Blechert, J., \& Tuschen-Caffier, B. (2009). Body-related film clip triggers desire to binge in women with binge eating disorder. Behaviour Research and Therapy, 47, 790-796. doi: 10.1016/j.brat.2009.06.005

Swinbourne, J., Hunt, C., Abbott, M., Russell, J., St Clare, T., \& Touyz, S. (2012). The comorbidity between eating disorders and anxiety disorders: Prevalence in an eating disorder sample and anxiety disorder sample. Australian and New Zealand Journal of Psychiatry, 46, 118-131. doi:10.1177/0004867411432071

Taylor, S., Koch, W. J., \& McNally, R. J. (1992). How does anxiety sensitivity vary across the anxiety disorders? Journal of Anxiety Disorders, 6, 249-259. doi:10.1016/0887$6185(92) 90037-8$

Taylor, S., Zvolensky, M. J., Cox, B. J., Deacon, B., Heimberg, R. G., Ledley, D. R., . . . Cardenas, S. J. (2007). Robust dimensions of anxiety sensitivity: Development and initial validation of the Anxiety Sensitivity Index-3. Psychological Assessment, 19, 176-188. doi:10.1037/1040-3590.19.2.176 
Utschig, A. C., Presnell, K., Madeley, M. C., \& Smits, J. A. (2010). An investigation of the relationship between fear of negative evaluation and bulimic psychopathology. Eating Behaviors, 11, 231-238. doi:10.1016/j.eatbeh.2010.05.003

van Beek, N., \& Griez, E. (2000). Reactivity to a $35 \% \mathrm{CO}_{2}$ challenge in healthy first-degree relatives of patients with panic disorder. Biological Psychiatry, 47, 830-835. doi:10.1016/S0006-3223(99)00265-6

van Hoeken, D., Veling, W., Sinke, S., Mitchell, J.E., Hoek, H.W. (2009). The validity and utility of sub-typing bulimia nervosa. International Journal of Eating Disorders, 42, 595602. 10.1002/eat.20724

Vander Wal, J. S., \& Thomas, N. (2004). Predictors of body image dissatisfaction and disturbed eating attitudes and behaviors in African American and Hispanic girls. Eating Behaviors, 5, 291-301. doi:10.1016/j.eatbeh.2004.04.001

Verburg, C., Griez, E., \& Meijer, J. (1994). A 35\% carbon dioxide challenge in simple phobias. Acta Psychiatrica Scandinavica, 90, 420-423. doi:10.1111/j.1600-0447.1994.tb01617.x

Verburg, K., Griez, E., Meijer, J., \& Pols, H. (1995). Discrimination between panic disorder and generalized anxiety disorder by $35 \%$ carbon dioxide challenge. American Journal of Psychiatry, 152, 1081-1083.

Verburg, K., Pols, H., de Leeuw, M., \& Griez, E. (1998). Reliability of the 35\% carbon dioxide panic provocation challenge. Psychiatry Research, 78, 207-214. doi:10.1016/S01651781(98)00009-2

Vickers, K., Jafarpour, S., Mofidi, A., Rafat, B., \& Woznica, A. (2012). The 35\% carbon dioxide test in stress and panic research: Overview of effects and integration of findings. Clinical Psychology Review, 32, 153-164. doi:10.1016/j.cpr.2011.12.004 
Vocks, S., Legenbauer, T., Wächter, A., Wucherer, M., \& Kosfelder, J. (2007). What happens in the course of body exposure? Emotional, cognitive, and physiological reactions to mirror confrontation in eating disorders. Journal of Psychosomatic Research, 62, 231-239. doi:10.1016/j.jpsychores.2006.08.007

Vohs, K. D., Bardone, A. M., Joiner, T. E., Jr., Abramson, L. Y., \& Heatherton, T. F. (1999). Perfectionism, perceived weight status, and self-esteem interact to predict bulimic symptoms: A model of bulimic symptom development. Journal of Abnormal Psychology, 108, 695-700. doi:10.1037/0021-843X.108.4.695

Vollrath, M. (2001). Personality and stress. Scandinavian Journal of Psychology, 42, 335-347. doi:10.1111/1467-9450.00245

Wallis, D. J., \& Hetherington, M. M. (2009). Emotions and eating. Self reported and experimentally induced changes in food intake under stress. Appetite, 52, 355-362. doi:10.1016/j.appet.2008.11.007

Wansink, B., Cheney, M. M., \& Chan, N. (2003). Exploring comfort food preferences across age and gender. Physiology \& Behavior, 79, 739-747. doi:10.1016/S0031-9384(03)00203-8

Watson, D., Clark, L. A., \& Carey, G. (1988). Positive and Negative Affectivity and their relation to anxiety and depressive disorder. Journal of Abnormal Psychology, 97, 346353.

Watson, D., \& Friend, R. (1969). Measurement of social-evaluative anxiety. Journal of Consulting and Clinical Psychology, 33, 448-457. doi:10.1037/h0027806

Wetherell, M. A., Crown, A. L., Lightman, S. L., Miles, J. N., Kaye, J., \& Vedhara, K. (2006). The four-dimensional stress test: Psychological, sympathetic-adrenal-medullary, 
parasympathetic and hypothalamic-pituitary-adrenal responses following inhalation of 35\% $\mathrm{CO}_{2}$. Psychoneuroendocrinology, 31, 736-747. doi:10.1016/j.psyneuen.2006.02.005

Wheaton, M. G., Deacon, B. J., McGrath, P. B., Berman, N. C., \& Abramowitz, J. S. (2012). Dimensions of anxiety sensitivity in the anxiety disorders: Evaluation of the ASI-3. Journal of Anxiety Disorders, 26, 401-408. doi:10.1016/j.janxdis.2012.01.002

Wheeler, L., \& Reis, H. T. (1991). Self-recording of everyday life events: Origins, types, and uses. Journal of Personality, 59, 339-354. doi:10.1111/j.1467-6494.1991.tb00252.x

Whiteside, U., Chen, E., Neighbors, C., Hunter, D., Lo, T., \& Larimer, M. (2007). Eating Behaviours, 8, 162-169. doi:10.1016/j.eatbeh.2006.04.001

Wilfley, D.E., Bishop, M.E., Wilson, G.T., \& Agras, W.S. (2007). Classification of eating disorders: toward DSM-5. International Journal of Eating Disorders, 40, 123-S129. 10.1002/eat.20436

Wilson, G. T., Grilo, C. M., \& Vitousek, K. M. (2007). Psychological treatment of eating disorders. Americal Psychologist, 62, 199-216. doi:10.1037/0003-066X.62.3.199

Wirtz, P. H., Elsenbruch, S., Emini, L., Rudisuli, K., Groessbauer, S., \& Ehlert, U. (2007). Perfectionism and the cortisol response to psychosocial stress in men. Psychosomatic Medicine, 69, 249-255. doi:10.1097/PSY.0b013e318042589e

Wonderlich, S. A., \& Mitchell, J. E. (1997). Eating disorders and comorbidity: empirical, conceptual, and clinical implications. Psychopharmacology Bulletin, 33, 381-390.

Woodside, D. B., Garfinkel, P. E., Lin, E., Goering, P., Kaplan, A. S., Goldbloom, D. S., \& Kennedy, S. H. (2001). Comparisons of men with full or partial eating disorders, men without eating disorders, and women with eating disorders in the community. American Jounral of Psychiatry, 158, 57-574. 
Woznica, A., Vickers, K., Koerner, N., \& Fracalanza, K. (2014). Mediators of response to carbon dioxide in bulimia nervosa and panic disorder. Department of Psychology, Ryerson University, Toronto, Canada.

Woznica, A., Vickers, K., Koerner, N., \& Fracalanza, K. (2015). Reactivity to carbon dioxide reactivity in bulimia nervosa and panic disorder. Manuscript submitted for publication.

Yang, H., \& Stober, J. (2012). The Physical Apperance Perfectionism Scale: Development and preliminary validation. Journal of Psychpathology and Behavioral Assessment, 34, 6983. doi:10.1007/210862-011-9260-7

Yeomans, M. R. \& Coughlan, E. (2009). Mood induced eating. Interactive effects of restraint and tendency to overeat. Appetite, 52, 290-298. doi:10.1016/j.appet.2008.10.006

Zvolensky, M. J., Bonn-Miller, M. O., Bernstein, A., McLeish, A. C., Feldner, M. T., \& LeenFeldner, E. W. (2006). Anxiety sensitivity interacts with marijuana use in the prediction of anxiety symptoms and panic-related catastrophic thinking among daily tobacco users. Behaviour Research and Therapy, 44, 907-924. doi:10.1016/j.brat.2005.06.005

Zvolensky, M. J., \& Eifert, G. H. (2001). A review of psychological factors/processes affecting anxious responding during voluntary hyperventilation and inhalations of carbon dioxideenriched air. Clinical Psychology Review, 21, 375-400. doi:10.1016/S02727358(99)00053-7 


\section{List of Abbreviations}

ACTH Adrenocorticotropic hormone

AN

Anorexia Nervosa

ANOVA Analysis of variance

APA American Psychiatric Association

AS

Anxiety Sensitivity

ASI-3 Anxiety Sensitivity Index - 3

BED Binge Eating Disorder

BFNE Brief Fear of Negative Evaluation

BIS Body Image Stressor

BMI Body Mass Index

BN Bulimia Nervosa

BPM Beats per minute

BSQ Body Shape Questionnaire

$\mathrm{CO}_{2} \quad$ Carbon dioixide

DASS-21-D Depression Anxiety Stress Scales - 21, depression subscale

DIA-BP Diastolic blood pressure

DSM Diagnostic and Statistical Manual of Mental Disorders

EAT-26 Eating Attitudes Test - 26

ED Eating Disorder

EDI Eating Disorder Inventory

EMA Ecological Momentary Assessment

FIR Finite impulse response 


\begin{tabular}{ll} 
FMPS & Frost Multidimensional Perfectionism Scale \\
FNE & Fear of Negative Evaluation \\
HC & Healthy control \\
HMPS & Hewitt and Flett Multidimesional Perfectionism Scale \\
HRSI & Holmes-Rahe Stress Inventory \\
HPA & Hypothalamic-pituitary-adrenal \\
HR & Heart rate \\
MEC & Maladaptive evaluative concerns \\
MDD & Major Depressive Disorder \\
MHQ & Medical History Questionniare \\
MINI 7.0 & Mini International Neuropsychiatric Interview 7.0 \\
NED & Non-eating disorder \\
OSFED & Other Specified Feeding or Eating Disorder \\
PAPS & Physical Appearance Perfectionism Scale \\
PD & Panic Disorder \\
PSL & Panic Symptoms List \\
PSS & Perceived Stress Scale \\
SAA & Social Appearance Anxiety \\
SAAS & Social Appearance Anxiety Scale \\
SAD & Social Anxiety Disorder \\
SAM & Sympathetic-adrenal-medullary \\
SD & Standard deviation \\
SOP & Self-oriented perfectionism \\
\hline
\end{tabular}


SPIN Social Phobia Inventory

STICSA State Trait Inventory for Cognitive and Somatic Anxiety

SYS-BP Systolic blood pressure

TSST Trier Social Stress Test

UFED Unspecified Feeding or Eating Disorder

VAS Visual analogue scale

WAI Worry about imperfection 